\title{
Molecular profiling of ovarian serous neoplasms : defining the borderline
}

Citation for published version (APA):

Bijsmans, I. T. G. W. (2011). Molecular profiling of ovarian serous neoplasms : defining the borderline. [Doctoral Thesis, Maastricht University]. Maastricht University. https://doi.org/10.26481/dis.20111021ib

Document status and date:

Published: 01/01/2011

DOI:

10.26481/dis.20111021ib

Document Version:

Publisher's PDF, also known as Version of record

\section{Please check the document version of this publication:}

- A submitted manuscript is the version of the article upon submission and before peer-review. There can be important differences between the submitted version and the official published version of record.

People interested in the research are advised to contact the author for the final version of the publication, or visit the DOI to the publisher's website.

- The final author version and the galley proof are versions of the publication after peer review.

- The final published version features the final layout of the paper including the volume, issue and page numbers.

Link to publication

\footnotetext{
General rights rights.

- You may freely distribute the URL identifying the publication in the public portal. please follow below link for the End User Agreement:

www.umlib.nl/taverne-license

Take down policy

If you believe that this document breaches copyright please contact us at:

repository@maastrichtuniversity.nl

providing details and we will investigate your claim.
}

Copyright and moral rights for the publications made accessible in the public portal are retained by the authors and/or other copyright owners and it is a condition of accessing publications that users recognise and abide by the legal requirements associated with these

- Users may download and print one copy of any publication from the public portal for the purpose of private study or research.

- You may not further distribute the material or use it for any profit-making activity or commercial gain

If the publication is distributed under the terms of Article $25 \mathrm{fa}$ of the Dutch Copyright Act, indicated by the "Taverne" license above, 
Molecular profiling of ovarian serous neoplasms: defining the borderline 
Molecular profiling of ovarian serous neoplasms: defining the borderline Ingrid T.G.W. Bijsmans

\section{ISBN:}

Cover design: Annemiek Hendrikx

Lay-out: $\quad$ Yvonne Leenders

Printed by: $\quad$ Wöhrmann Print Service

(C) Copyright I.T.G.W. Bijsmans, Maastricht 2011

All rights reserved. No part of this thesis may be reproduced or transmitted in any form or by any means, electronic or mechanical, including photocopying, recording or any information storage or retrieval system, without permission in writing from the author, or, when appropriate, from the publishers of the publications. 


\title{
Molecular profiling of ovarian serous neoplasms: defining the borderline
}

\author{
PROEFSCHRIFT \\ Ter verkrijging van de graad van doctor \\ aan de Universiteit Maastricht, \\ op gezag van de Rector Magnificus, \\ Prof. mr. G.P.M.F. Mols, \\ volgens het besluit van het college van Decanen, \\ in het openbaar te verdedigen \\ op vrijdag 21 oktober 2011 om 10.00 uur
}

door

Ingrid Tine Gerarda Wilhelmina Bijsmans

Geboren op 21 december 1979

te Heerlen 


\section{Promotoren:}

Prof. dr. A.P. de Bruïne

Prof. dr. M. van Engeland

\section{Copromotoren:}

Dr. N.L.G. Sieben

Dr. K.K. Van de Vijver

\section{Beoordelingscommissie:}

Prof. dr. F.C.S. Ramaekers (voorzitter)

Prof. dr. G.J. Fleuren (Leiden Universitair Medisch Centrum)

Prof. dr. R.F.P.M. Kruitwagen

Dr. R.I. Lalisang

Dr. E.M.D. Schuuring (Universitair Medisch Centrum Groningen)

\section{greiner bio-one MD*Health}

Financial support by Amgen B.V., Dako Benelux, Greiner Bio-One, MDx Health, Novartis Oncology, and Roche Nederland B.V. for publication of this thesis is gratefully acknowledged 
Do not go where the path may lead, go instead where there is no path and leave a trail

Ralph Waldo Emerson 
List of abbreviations

\begin{tabular}{|c|c|}
\hline APST & atypical proliferative serous tumor \\
\hline $\mathrm{CGH}$ & comparative genomic hybridization \\
\hline $\mathrm{Cl}$ & confidence interval \\
\hline CIMP & CpG island methylator phenotype \\
\hline CNA & copy number alteration \\
\hline CRC & colorectal cancer \\
\hline DAC & 5-aza-2'-deoxycytidine \\
\hline ECM & extracellular matrix \\
\hline FDR & false discovery rate \\
\hline FIGO & International Federation of Gynecology and Obstetrics \\
\hline FISH & fluorescence in situ hybridization \\
\hline FT & fallopian tube \\
\hline FTC & fallopian tube carcinoma \\
\hline Hcy & homocysteine \\
\hline HG-SCA & high-grade serous carcinoma \\
\hline $\mathrm{HR}$ & hazard ratio \\
\hline LG-SCA & low-grade serous carcinoma \\
\hline LMP & tumor of low malignant potential \\
\hline $\mathrm{LOH}$ & loss of heterozygosity \\
\hline MLPA & multiplex ligation-dependent probe amplification \\
\hline MMP & matrix metallo protease \\
\hline MP-SBT & micropapillary serous borderline tumor \\
\hline MPSC & micropapillary serous carcinoma \\
\hline MSP & methylation specific PCR \\
\hline ND & not determined \\
\hline NE & not expressed \\
\hline NT & next tier \\
\hline RTK & receptor tyrosine kinase \\
\hline RT-PCR & reverse transcriptase polymerase chain reaction \\
\hline SAM & significance analysis of microarrays \\
\hline SBT & serous borderline tumor \\
\hline SCA & serous carcinoma \\
\hline SCA1 & grade 1 SCA \\
\hline SCA2 & grade 2 SCA \\
\hline SCA3 & grade 3 SCA \\
\hline SEM & standard error of mean \\
\hline SNP & single nucleotide polymorphism \\
\hline TN & terminal node \\
\hline TSA & trichostatin A \\
\hline TSG & tumor suppressor gene \\
\hline TSS & transcription start site \\
\hline TT & top tier \\
\hline uPA & urokinase-type plasminogen activator \\
\hline WHO & World Health Organization \\
\hline WT & wild-type \\
\hline
\end{tabular}




\section{CONTENTS}

Chapter $1 \quad$ General introduction $\quad 9$

Chapter 2 Loss of SerpinA5 protein expression is associated with advanced- 25 stage serous ovarian tumors

Chapter 3 E2Fs mediate a fundamental cell cycle deregulation in high-grade serous ovarian carcinomas

Chapter 4 Decision tree analysis of copy number alterations identifies MECOM, LIMK1, NFKBIE, ACTN4, and NCOA3 as discriminators of serous ovarian borderline tumors and carcinomas

Chapter 5 Identification of candidate tumor suppressor genes in ovarian serous borderline tumors and carcinomas: an epigenetic approach

Chapter 6 Betaine-homocysteine methyltransferase 2 (BHMT2) is a candidate 95 tumor suppressor gene frequently inactivated by promoter CpG island hypermethylation in serous ovarian cancer

Chapter 7 General discussion

Summary

Samenvatting

Dankwoord

129

Curriculum vitae 



\section{CHAPTER 1}

General introduction 
Ovarian cancer accounts for approximately $4 \%$ of all new cancer cases in women and is a leading cause of cancer-related deaths among women with gynecologic malignancies [1]. Worldwide, an estimated 225,000 new cases and 140,000 deaths were reported in 2008 [2]. In the same year, the annual estimated cases and deaths were 45,000 and 29,000 within the European Union [3], and 1,200 and 1,000 respectively in The Netherlands [4]. The high mortality is related to the fact that in most cases the disease has already spread to the upper abdomen at time of presentation. The incidence of ovarian cancer increases with age, with most tumors occurring after menopause [1].

\section{Ovarian cancer is a heterogeneous disease}

Ovarian tumors develop from three cell types: epithelial cells, sex cord-stromal cells or germ cells (oocytes). Epithelial-derived tumors account for $90 \%$ of ovarian cancers and represent the most lethal form [5]. Based on histology four major ovarian surface epithelial tumors are known: serous, mucinous, endometrioid, and clear cell tumors. These different subtypes are associated with differences in incidence and underlying molecular abnormalities (Table 1 and Figure 1). Surface epithelial ovarian tumors can be divided into benign, borderline or malignant based on architecture, nuclear atypia and stromal-invasive features. A combination of the above mentioned criteria, with respect to molecular mechanisms, precursor lesions, patterns of spread, long-time follow-up, therapeutic response and prognostic factors, has led to the development of a model subdividing these ovarian tumors in type I and type II tumors [6]. This model corresponds to different pathways of tumorigenesis.

Table 1: Main characteristics of ovarian surface epithelial tumors

\begin{tabular}{|c|c|c|c|}
\hline Subtype & Entity & Abnormalities & References \\
\hline serous $(53 \%)$ & $\begin{array}{l}\text { benign }(60-65 \%) \\
\text { borderline }(10 \%) \\
\text { malignant }(25-30 \%)\end{array}$ & $\begin{array}{l}\text { BRAF or KRAS mutations }(60 \%) \\
\text { TP53 mutations (up to } 80 \%) \\
\text { BRCA1/2 dysfunction* }(5-53 \%)\end{array}$ & $\begin{array}{l}{[5,7,8]} \\
{[5,9,10]} \\
{[11-15]}\end{array}$ \\
\hline mucinous (31\%) & $\begin{array}{l}\text { benign }(80 \%) \\
\text { borderline }(5-10 \%) \\
\text { malignant }(10-15 \%)\end{array}$ & $\begin{array}{l}\text { KRAS mutations }(48-79 \%) \\
\text { KRAS mutations }(50-75 \%)\end{array}$ & $\begin{array}{l}{[5,7,16,17]} \\
{[5,7,16,17]}\end{array}$ \\
\hline endometrioid (6\%) & $\begin{array}{l}\text { benign }(5 \%) \\
\text { borderline }(20 \%) \\
\text { malignant }(75 \%)\end{array}$ & $\begin{array}{l}\text { LOH PTEN }(43-75 \%) \\
\text { PTEN mutations }(20-31 \%) \\
\text { CTNNB1 mutations }(24 \%)\end{array}$ & {$[5,18-22]$} \\
\hline clear cell $(3 \%)$ & $\begin{array}{l}\text { borderline }(2 \%) \\
\text { malignant }(98 \%)\end{array}$ & $\begin{array}{l}\text { PIK3CA mutations }(25-33 \%) \\
\text { LOH PTEN }(14-36 \%)\end{array}$ & {$[5,18-20,22,23]$} \\
\hline
\end{tabular}

Type I tumors include mucinous, endometrioid, transitional cell (including Brenner tumors), and low-grade serous tumors. Type II tumors constitute high-grade serous carcinomas, undifferentiated carcinomas and malignant mixed mesodermal tumors (carcinosarcomas). Type II tumors usually present in a more advanced stage, representing more aggressive tumors. Clear cell tumors have features of both type I and type II tumors. The main problem in the clinical management of adnexal masses is the risk of malignancy. Therefore, the clinically most relevant classification is the division into benign, borderline and malignant. 
General introduction

Insert Figure 1 


\section{Risk factors}

An established risk factor for ovarian cancer is a positive family history. The two most common hereditary cancer syndromes associated with ovarian cancer are Hereditary Breast and Ovarian Cancer (HBOC) and Hereditary Non-Polyposis Colorectal Cancer (HNPCC), also known as Lynch syndrome. Hereditary ovarian cancer accounts for at least $10 \%$ of all cases [24]. Approximately $90 \%$ of the ovarian cancers in the HBOC syndrome is attributable to mutations in the caretaker genes BRCA1 and BRCA2, of which $B R C A 1$ accounts for the majority of mutations [24, 25]. Ovarian cancer accounts for $10 \%$ of HNPCC patients. Mutations in the mismatch repair genes $M L H 1, M S H 2$, MSH6 and PMS2 are associated with ovarian cancers in HNPCC [25]. Factors that have been associated with a decreased risk of ovarian cancer include late menarche, use of oral contraceptives, child bearing, breast feeding, tubal ligation or hysterectomy, and early menopause $[1,26-30]$. The risk of ovarian cancer increases with the number of ovulations, presumably through increased DNA damage inducing e.g. TP53 mutations [31]. Lifestyle factors such as cigarette smoking and alcohol consumption showed controversial results [32-34].

\section{Diagnosis}

Because women generally do not have complaints, the disease has often spread beyond the ovaries at time of presentation. The symptoms of ovarian cancer are usually non-specific. The most common symptoms are abdominal pain, bloating, and urinary or gastrointestinal tract symptoms. A palpable ovarian mass is typically found by physical examination. Other findings may include ascites, pleural effusions and Sister Mary Joseph's nodule, or an umbilical mass. Transvaginal ultrasonography is often performed for further evaluation of the pelvis if ovarian cancer is suspected on the basis of symptoms and physical examination [35]. Although not sufficiently sensitive and specific, CA-125 is elevated in more than $80 \%$ of advanced-stage epithelial ovarian cancer. Surgery is generally necessary for definite diagnosis [35].

\section{Staging and treatment}

Ovarian malignancies are commonly staged using the International Federation of Gynecology and Obstetrics (FIGO) staging system. In The Netherlands, a staging procedure including bilateral salpingo-oophorectomy, peritoneal biopsies and lymph node sampling is performed in early-stage (FIGO la-Ila) ovarian carcinomas. Optimal staging and tumor grade are the most important prognostic factors for overall and recurrence-free survival in the early-stage ovarian carcinomas [36, 37]. The standard treatment of advanced-stage (FIGO IIb-IV) ovarian carcinoma is primary surgical debulking followed by taxane- and platinum-based chemotherapy [36]. Whereas the majority of patients respond to chemotherapy at first instance, most patients will recur [38]. The amount of residual tumor after cytoreductive surgery is inversely correlated with progression-free and overall survival [39]. Treatment of borderline tumors consists of surgical resection of the tumor and careful inspection of the peritoneal cavity. Treatment by uni- or bilateral salpingo-oophorectomy depends on the age and child-wish of premenopausal women, whereas bilateral resection is offered to postmenopausal women [40]. 


\section{Prognosis}

Long-term survival rates among patients with early-stage carcinoma range from 80 to $90 \%$, whereas advanced-stage patients have survival rates of 10 to $30 \%$. Although borderline tumor patients may present with implants (tumor deposits in the peritoneal cavity), the 5-year survival remains excellent (90-95\%) [5].

\section{Serous ovarian tumors}

Serous ovarian tumors are the most frequently occurring surface epithelial tumors. Benign serous tumors occur in more than $60 \%$ and can occur at any age, being most common in the reproductive age group ( $<50$ years old). Most tumors are found incidentally, but sometimes patients present with nonspecific signs and symptoms including lower abdominal pain or enlargement [41, 42]. Serous borderline tumors (SBTs) belong to an intermediate group of ovarian tumors, characterized by stratified epithelial cells, varying nuclear atypia, and lack of destructive stromal invasion $[5,43]$. These tumors occur mostly in the fourth or fifth decade of life, with a mean age at diagnosis of 46 years [44]. The incidence of serous carcinomas (SCAs) slightly increases from the second decade of life, the mean age at diagnosis is 56 years [44].

Serous tumors demonstrate a broad spectrum of growth patterns [22]. This morphologic heterogeneity is likely an expression of the high genetic and/or epigenetic variation. The distinctive growth patterns include extensive and often complex papillarity with prominent cellular budding, formation of glands and cribriform structures with irregular slit-like lumens, microcysts and solid nests and sheets [22, 42, 45-47]. Psammoma bodies and larger calcific deposits are common. SCAs are graded using a 3-tier system into well, moderately and poorly differentiated tumors, based on architectural features, nuclear atypia and mitotic index [48]. A 2-tier system has also been proposed which uses cytologic atypia and mitotic activity [49]. Recently, a model based on morphology and the underlying biology was proposed to divide SCAs into low (LG) and high-grade (HG) tumors [7, 50, 51]. Low-grade carcinomas account for only $5 \%$ of all ovarian tumors and are thought to develop slowly and stepwise from benign cystadenomas and SBTs, in an adenoma-carcinoma sequence. A controversy exists about the development of HG-SCAs and whether SBTs are precursors of SCAs. It has been described that high-grade carcinomas are thought to originate outside the ovaries, namely in the fallopian tube fimbriae [6,52] or the endometrium [53]. Previous work in our group by Sieben et al. indicated that SBTs and SCAs are genetically different [7], whereas another study suggested that high-grade carcinomas may arise from a low-grade carcinoma or a borderline tumor, although very infrequently [54].

\section{Molecular biology of SBTs and SCAs}

Knowledge about gene expression [55-60], genetic and epigenetic abnormalities in serous ovarian cancer increased the last decades. Ovarian tumorigenesis is characterized by mutations in several key genes. Frequently altered signaling pathways are described. 


\section{RAS-RAF-MEK-ERK-MAP kinase (MAPK) signaling}

MAPK signaling involves binding of growth factors to receptor tyrosine kinases (RTKs) to activate the small G-protein $R A S$ and the downstream serine-threonine-specific protein kinase RAF. RAF activates MEK, which subsequently activates ERK and its downstream targets, leading to proliferation and transformation [61, 62]. BRAF and KRAS are two upstream key players in this pathway, which are frequently mutated in SBTs $[7,8,16,17,50,63,64]$, while mutations are absent (BRAF) or hardly detected (KRAS) in SCAs $[7,17,64]$. Destructive stromal invasion by MAPK signaling is inhibited by the ERK-inhibitor DUSP4 and the uPA-inhibitor SerpinA5 in SBTs, whereas this inhibition has been lost in SCAs due to downregulation of DUSP4 and SerpinA5 [59].

\section{TP53 and Rb/E2F signaling}

The tumor suppressor gene (TSG) TP53 has been described as the 'guardian of the genome' $[65,66]$. Activation of the TP53 protein occurs after DNA damage, aberrant growth signaling, or can be induced by chemotherapeutic regimens [67]. TP53 activation leads to DNA binding, expression of target genes, thereby initiating processes that ultimately block proliferation, induce apoptosis and inhibit cell cycle progression $[65,67]$. In normal cells, TP53 is present in low concentrations due to a feedback loop with MDM2. MDM2 activity can be inhibited by $\mathrm{p} 14^{\mathrm{ARF}}$, after its stimulation by oncogenes [67]. Inactivation of the TP53 pathway results from deletion of chromosome $17 p$, TP53 mutations, MDM2 overexpression, or $p 14^{A R F}$ promoter hypermethylation [68]. Missense TP53 mutations are mainly identified in exons 5 to 8 (DNA binding domain) and are detected in up to $80 \%$ of SCAs, whereas rarely present $(0-8 \%)$ in SBTs $[9,51,69-71]$. Overexpression of mutant TP53 protein has been detected in over $50 \%$ of SCAs [16, 71-73]. Overexpression of MDM2 was not found in normal tissue and SBTs, while stage I SCAs showed overexpression in 9\% [74]. Locus 9p21, harboring the TSGs $p 14^{A R F}$ and $p 16^{I N K 4 A}$, is deleted in $28-70 \%$ of SCAs $[75,76]$. Methylation of $p 14^{A R F}$ or $p 16^{I N K 4 A}$ is not a frequently occurring event in (serous) ovarian cancer [77-79]. Disruption of the Rb/E2F pathway is a common feature in human cancers including ovarian cancer. $R b 1$ functions as a G1 checkpoint to maintain cell cycle arrest [80]. Loss of heterozygosity ( $\mathrm{LOH})$ of $R b 1$ is absent in benign tissue and SBTs, but present in $24 \%$ of SCAs [81]. Cyclins with a pronounced role in serous ovarian cancer are CCND1 and CCNE1. Overexpression of cyclin D1 is an independent prognostic marker of overall survival in SCA patients [73]. Increased CCNE1 expression or amplification are associated with poorly differentiated tumors, higher grade, advanced-stage, poor prognosis, and survival [73, 82, 83]. Cyclin E1 overexpression is detected in $20-45 \%$ of SCAs $[65,74,75]$, CCNE1 amplifications have been detected in $54 \%$ of SCAs [84] and are associated with chemo resistance in SCAs [85]. Eight members of the $E 2 F$ family have been identified thus far: the proliferation promoting genes $E 2 F 1, E 2 F 2$ and $E 2 F 3 a$; the transcriptional repressors $E 2 F 3 b, E 2 F 4$ and $E 2 F 5$; and $E 2 F 6, E 2 F 7$, and E2F8 of which the function is less clear [86]. High E2F1/E2F2/E2F8 expression has been associated with high tumor grade and residual disease. Overexpression of E2F1 and E2F2 is associated with shorter disease-free and overall survival in ovarian tumors [86]. E2F1 has a dual role in ovarian cancer because this gene can either promote proliferation or induce apoptosis [86-89]. Expression of the cell cycle inhibitor CDKN1A is significantly higher in SBTs than in SCAs $[55,57,58]$. 
CDKN1A protein expression was either low or absent in 77\% of SCAs [73], associated with high-grade, advanced-stage, poor survival and shorter progression-free survival $[73,90]$.

\section{BRCA1/BRCA2 pathway}

$B R C A 1$ and $B R C A 2$ are considered to be 'caretakers' of the genome, maintaining chromosomal and DNA stability [91]. Both genes are involved in regulation of cellular proliferation and DNA repair by homologous recombination. BRCA1 also functions in checkpoint control, ubiquitination in response to replication stress and chromatin remodeling, a process which occurs around double strand DNA breaks, thought to perform DNA repair $[92,93]$. Both genetic and epigenetic mechanisms are involved in the inactivation of the BRCA genes [94]. LOH of the BRCA1 locus was detected in $53 \%$ of SCAs [12]. In a population-based setting, BRCA1 and BRCA2 were not mutated in borderline tumors, whereas SCAs harbored mutations in up to $12 \%$ and $8 \%$ respectively $[13,15,95]$. BRCA2 promoter hypermethylation has been detected in $8 \%$ of ovarian tumors [13].

\section{PI3K/AKT pathway}

Phosphatidylinositol 3'-kinases (PI3Ks) are intracellular lipid kinases. Growth factor RTKs activate PI3Ks via the regulatory subunit p85. Interaction with the catalytic subunit $\mathrm{p} 110 \alpha(P I K 3 C A)$ results in an active PI3K heterodimer $[96,97]$. Activated PI3K phosphorylates phosphatidylinositol-4,5-bisphosphate $\left(\mathrm{PIP}_{2}\right)$ to generate phosphatidylinositol-3,4,5-trisphosphate $\left(\mathrm{PIP}_{3}\right)$. Binding of $\mathrm{PIP}_{3}$ to AKT activates the latter thereby triggering growth regulation, cell cycle progression, proliferation, and cell survival $[96,98]$. Phosphatase and tensin homolog deleted on chromosome 10 (PTEN) belongs to the family of protein-tyrosine phosphatases, a class of kinases with dual-specificity phosphatase activity. PTEN dephosphorylates $\mathrm{PIP}_{3}$ thereby regulating the PI3K/AKT pathway [99]. PTEN was recently named 'new guardian of the genome', since PTEN protects against genomic instability by other mechanisms than TP53 [100]. PI3Ks function as oncogenes in ovarian cancer. PIK3CA overexpression led to increased transcription, vascular endothelial growth factor (VEGF) expression, protein expression and PI3K activity, resulting in increased angiogenesis and reduced apoptosis [97, 101]. Comparative genomic hybridization (CGH) and single nucleotide polymorphism (SNP) array analysis identified chromosomal gains of PIK3CA in up to $40 \%$ of HG-SCAs [102, 103]. Amplifications of PIK3CA were absent in normal tissue and SBTs, but have been detected in $13-30 \%$ of SCAs, depending on the technique used [104, 105]. Activating PIK3CA mutations are rarely detected in SBTs (5\%) and SCAs (<3\%) [104-106]. AKT2 is a downstream target of $P I 3 K$ and belongs to the serine/threonine kinase subfamily. HGSCAs showed AKT2 amplification in $18 \%$ of cases, this aberration is absent in normal tissue and SBTs. Mutations have not been identified in normal tissue or serous tumors [105]. Taken together, these data indicate that genetic alterations of PIK3CA and AKT are not frequently involved in pathway deregulation in serous ovarian cancer. LOH of $10 \mathrm{q}$, the region where PTEN is located, was detected in $28-40 \%$ of SCAs $[18,19,107]$. Mutations are rarely $(<10 \%)$ detected in SCAs $[18,19,108]$ and have thus far not been assessed in SBTs. 


\section{EGFR signaling}

The human epidermal growth factor receptor (EGFR) family consists of the four tyrosine kinase receptors EGFR (HER-1), ERBB2 (HER-2), ERBB3 (HER-3) and ERBB4 (HER-4) [109, 110]. EGFR is un upstream activator of PI3K [98]. The EGFR locus is gained in $44 \%$ of SCAs [109], mutations are rare $[60,109,111]$. Statistically significant associations with tumor grade, residual disease, age, shorter overall survival, and disease-free survival, were detected in patients with increased EGFR copy numbers and protein overexpression [109]. As for other members in this pathway, HER-2 mutations are rare $[60,105,109]$.

Crosstalk between the pathways has been described. For example, TP53 can negatively regulate PI3K activity. TP53 interacts directly with the PI3K promoter, thereby decreasing PIK3CA transcription and activity of (non)malignant ovarian epithelial cells. Vice versa, loss of TP53 increases PIK3CA expression [112]. PI3K signaling can protect cells from TP53 induced apoptosis [113].

\section{Scope and outline of this thesis}

The aim of this thesis was to investigate whether SBTs can be distinguished from SCAs. The distinction between SBTs and SCAs is a common problem in ovarian cancer pathology. From a clinical point of view, the histological differentiation is important, since both patient management and prognosis differ substantially. Additional tools to assist pathologists in making the differential diagnosis could aid better tumor classification. Immunohistochemical and molecular biological approaches were performed in this respect. Our previous microarray study revealed that although the MAPK pathway is activated in SBTs, activation of downstream genes involved in extracellular matrix degradation is absent, suggesting an uncoupling of these events. One of the genes involved in this uncoupling is the UPA-inhibitor SerpinA5, which was downregulated in SCAs as compared to SBTs. Loss of SerpinA5 mRNA expression in SCAs was associated with downstream activation of the matrix metalloproteinase 9 (MMP9) followed by degradation of the extracellular matrix and invasion. SerpinA5 was proposed as a putative TSG providing evidence for the indolent behavior of SBTs [44]. In Chapter 2, SerpinA5 protein expression was determined by immunohistochemistry in SBTs, SCAs and omental metastases. Cell cycle deregulation at the mRNA level was studied in more detail in Chapter 3. In Chapter 4, we investigated if SBTs and SCAs can be distinguished based on DNA copy number alterations as determined by multiplex ligation-dependent probe amplification (MLPA) analysis. In addition to genetic alterations, epigenetic regulation frequently alters gene expression. Promoter CpG island hypermethylation is the best characterized and most studied epigenetic alteration in cancer. Chapter $\mathbf{5}$ describes the identification of novel TSGs inactivated by promoter CpG island hypermethylation that discriminate between SBTs and SCAs and eventually might serve as diagnostic tools. Three approaches were used: 1) a candidate gene approach (literature-based genes reported to be frequently methylated in other cancers); 2) promoter sequence (genome-wide promoter alignment in combination with specific sequence motifs in the promoter region of $\mathrm{CpG}$ islands); and 3) pharmacological unmasking of hypermethylated TSGs to reactivate transcriptional silenced genes. The potential TSG BHMT2 (Chapter 6) was identified by 
applying the pharmacological approach. The general discussion in Chapter 7 summarizes the major findings of the studies presented in this thesis, followed by suggestions for diagnostic and clinical applications of the identified genes and future directions. 


\section{References}

1. Marmot M. World Cancer Research Fund / American Institute for Cancer Research. Food, Nutrition, Physical Activity, and the Prevention of Cancer: a Global Perspective. Washington DC: American Institute for Cancer Research; 2009.

2. Ferlay J, Shin HR, Bray F, Forman D, Mathers C, Parkin DM. Estimates of worldwide burden of cancer in 2008: GLOBOCAN 2008. Int J Cancer 2010;127(12):2918-27.

3. Ferlay J, Parkin DM, Steliarova-Foucher E. Estimates of cancer incidence and mortality in Europe in 2008. Eur J Cancer. Eur J Cancer. 2010;46(4):765-81.

4. Registration DC. Cancer incidence and deaths in the Netherlands. 2008 [cited; Available from: http://www.ikcnet.nl/page.php?id=160

5. Prat J. Surface epithelial-stromal tumors: Serous tumors: Saunders; 2004: 83-111

6. Shih le M, Kurman RJ. Ovarian tumorigenesis: a proposed model based on morphological and molecular genetic analysis. Am J Pathol 2004;164(5):1511-8.

7. Sieben NL, Macropoulos P, Roemen GM, et al. In ovarian neoplasms, BRAF, but not KRAS, mutations are restricted to low-grade serous tumours. J Pathol 2004;202(3):336-40.

8. Singer $\mathrm{G}$, Oldt $\mathrm{R}$, 3rd, Cohen $\mathrm{Y}$, et al. Mutations in BRAF and KRAS characterize the development of low-grade ovarian serous carcinoma. J Natl Cancer Inst 2003;95(6):484-6.

9. Salani R, Kurman RJ, Giuntoli R, 2nd, et al. Assessment of TP53 mutation using purified tissue samples of ovarian serous carcinomas reveals a higher mutation rate than previously reported and does not correlate with drug resistance. Int J Gynecol Cancer 2008;18(3):48791.

10. Shih le M, Kurman RJ. Molecular pathogenesis of ovarian borderline tumors: new insights and old challenges. Clin Cancer Res 2005;11(20):7273-9.

11. Foster KA, Harrington P, Kerr J, et al. Somatic and germline mutations of the BRCA2 gene in sporadic ovarian cancer. Cancer Res 1996;56(16):3622-5.

12. Geisler JP, Hatterman-Zogg MA, Rathe JA, Buller RE. Frequency of BRCA1 dysfunction in ovarian cancer. J Natl Cancer Inst 2002;94(1):61-7.

13. Hilton JL, Geisler JP, Rathe JA, Hattermann-Zogg MA, DeYoung B, Buller RE. Inactivation of BRCA1 and BRCA2 in ovarian cancer. J Natl Cancer Inst 2002;94(18):1396-406.

14. Lancaster JM, Wooster R, Mangion J, et al. BRCA2 mutations in primary breast and ovarian cancers. Nat Genet 1996;13(2):238-40.

15. Pal T, Permuth-Wey J, Betts JA, et al. BRCA1 and BRCA2 mutations account for a large proportion of ovarian carcinoma cases. Cancer 2005;104(12):2807-16.

16. Caduff RF, Svoboda-Newman SM, Ferguson AW, Johnston CM, Frank TS. Comparison of mutations of Ki-RAS and p53 immunoreactivity in borderline and malignant epithelial ovarian tumors. Am J Surg Pathol 1999;23(3):323-8.

17. Mok SC, Bell DA, Knapp RC, et al. Mutation of K-ras protooncogene in human ovarian epithelial tumors of borderline malignancy. Cancer Res 1993;53(7):1489-92.

18. Obata K, Morland SJ, Watson RH, et al. Frequent PTEN/MMAC mutations in endometrioid but not serous or mucinous epithelial ovarian tumors. Cancer Res 1998;58(10):2095-7.

19. Saito $\mathrm{M}$, Okamoto $\mathrm{A}$, Kohno $\mathrm{T}$, et al. Allelic imbalance and mutations of the PTEN gene in ovarian cancer. Int J Cancer 2000;85(2):160-5.

20. Willner J, Wurz $\mathrm{K}$, Allison $\mathrm{KH}$, et al. Alternate molecular genetic pathways in ovarian carcinomas of common histological types. Human pathology 2007;38(4):607-13.

21. Catasus L, Bussaglia E, Rodrguez I, et al. Molecular genetic alterations in endometrioid carcinomas of the ovary: similar frequency of beta-catenin abnormalities but lower rate of microsatellite instability and PTEN alterations than in uterine endometrioid carcinomas. Human pathology 2004;35(11):1360-8.

22. Soslow RA. Histologic subtypes of ovarian carcinoma: an overview. Int J Gynecol Pathol 2008;27(2):161-74. 
23. Kuo KT, Mao TL, Jones $\mathrm{S}$, et al. Frequent activating mutations of PIK3CA in ovarian clear cell carcinoma. Am J Pathol 2009;174(5):1597-601.

24. Prat J, Ribe A, Gallardo A. Hereditary ovarian cancer. Human pathology 2005;36(8):861-70.

25. Lynch HT, Casey MJ, Snyder CL, et al. Hereditary ovarian carcinoma: heterogeneity, molecular genetics, pathology, and management. Mol Oncol 2009;3(2):97-137.

26. Booth $M$, Beral V, Smith P. Risk factors for ovarian cancer: a case-control study. British journal of cancer 1989;60(4):592-8.

27. Adami HO, Hsieh CC, Lambe $\mathrm{M}$, et al. Parity, age at first childbirth, and risk of ovarian cancer. Lancet 1994;344(8932):1250-4.

28. Whittemore AS, Harris R, Itnyre J. Characteristics relating to ovarian cancer risk: collaborative analysis of 12 US case-control studies. II. Invasive epithelial ovarian cancers in white women. Collaborative Ovarian Cancer Group. American journal of epidemiology 1992;136(10):1184-203.

29. Miracle-McMahill HL, Calle EE, Kosinski AS, et al. Tubal ligation and fatal ovarian cancer in a large prospective cohort study. American journal of epidemiology 1997;145(4):349-57.

30. Hankinson SE, Hunter DJ, Colditz GA, et al. Tubal ligation, hysterectomy, and risk of ovarian cancer. A prospective study. Jama 1993;270(23):2813-8.

31. Schildkraut JM, Bastos E, Berchuck A. Relationship between lifetime ovulatory cycles and overexpression of mutant p53 in epithelial ovarian cancer. J Natl Cancer Inst 1997;89(13):932-8.

32. Chang ET, Canchola AJ, Lee VS, et al. Wine and other alcohol consumption and risk of ovarian cancer in the California Teachers Study cohort. Cancer Causes Control 2007;18(1):91-103.

33. Marchbanks PA, Wilson H, Bastos E, Cramer DW, Schildkraut JM, Peterson HB. Cigarette smoking and epithelial ovarian cancer by histologic type. Obstet Gynecol 2000;95(2):25560.

34. Riman T, Dickman PW, Nilsson S, Nordlinder H, Magnusson CM, Persson IR. Some life-style factors and the risk of invasive epithelial ovarian cancer in Swedish women. Eur J Epidemiol 2004;19(11):1011-9.

35. Cannistra SA. Cancer of the ovary. N Engl J Med 2004;351(24):2519-29.

36. Epitheliaal ovariumcarcinoom; landelijke richtlijn. the Netherlands; 2009.

37. Trimbos $B$, Timmers $\mathrm{P}$, Pecorelli $\mathrm{S}$, et al. Surgical staging and treatment of early ovarian cancer: long-term analysis from a randomized trial. J Natl Cancer Inst 2010;102(13):982-7.

38. Ozols RF. Systemic therapy for ovarian cancer: current status and new treatments. Seminars in oncology 2006;33(2 Suppl 6):S3-11.

39. Fader AN, Rose PG. Role of surgery in ovarian carcinoma. J Clin Oncol 2007;25(20):2873-83.

40. Borderline ovariumtumoren; landelijke richtlijn. the Netherlands; 2010.

41. Lee KR, Prat J et al. Tumours of the ovary and peritoneum. In: Tavassoli FA DP, editor. Pathology and genetics of tumours of the breast and female genital organs: Lyon: IAEC press; 2004. p. 113-203.

42. Scully RE Young R, Clement PB. Surface epithelial-stromal tumors; serous tumors. In: Rosai J $\mathrm{SL}$, editor. Atlas of tumor pathology Tumors of the ovary, maldeveloped gonads, fallopian tube, and broad ligament; 1998. p. 51-79.

43. Crispens MA. Borderline ovarian tumours: a review of the recent literature. Curr Opin Obstet Gynecol 2003;15(1):39-43.

44. Gilks TLaCB. Gynecologic Pathology: Elsevier Churchill Livingstone; 2009.

45. Eichhorn JH, Bell DA, Young RH, Scully RE. Ovarian serous borderline tumors with micropapillary and cribriform patterns: a study of 40 cases and comparison with 44 cases without these patterns. Am J Surg Pathol 1999;23(4):397-409.

46. McCluggage WG. My approach to and thoughts on the typing of ovarian carcinomas. Journal of clinical pathology 2008;61(2):152-63. 
47. Seidman JD, Horkayne-Szakaly I, Haiba M, Boice CR, Kurman RJ, Ronnett BM. The histologic type and stage distribution of ovarian carcinomas of surface epithelial origin. Int J Gynecol Pathol 2004;23(1):41-4.

48. Silverberg SG. Histopathologic grading of ovarian carcinoma: a review and proposal. Int J Gynecol Pathol 2000;19(1):7-15.

49. Malpica A, Deavers MT, Tornos C, et al. Interobserver and intraobserver variability of a two-tier system for grading ovarian serous carcinoma. Am J Surg Pathol 2007;31(8):116874.

50. Ho CL, Kurman RJ, Dehari R, Wang TL, Shih le M. Mutations of BRAF and KRAS precede the development of ovarian serous borderline tumors. Cancer Res 2004;64(19):6915-8.

51. Singer G, Stohr R, Cope L, et al. Patterns of p53 mutations separate ovarian serous borderline tumors and low- and high-grade carcinomas and provide support for a new model of ovarian carcinogenesis: a mutational analysis with immunohistochemical correlation. Am J Surg Pathol 2005;29(2):218-24.

52. Levanon K, Crum C, Drapkin R. New insights into the pathogenesis of serous ovarian cancer and its clinical impact. J Clin Oncol 2008;26(32):5284-93.

53. Massuger L, Roelofsen T, Ham M, Bulten J. The origin of serous ovarian cancer may be found in the uterus: a novel hypothesis. Medical hypotheses 2010;74(5):859-61.

54. Dehari R, Kurman RJ, Logani S, Shih le M. The development of high-grade serous carcinoma from atypical proliferative (borderline) serous tumors and low-grade micropapillary serous carcinoma: a morphologic and molecular genetic analysis. Am J Surg Pathol 2007;31(7):1007-12.

55. Bonome T, Lee JY, Park DC, et al. Expression profiling of serous low malignant potential, low-grade, and high-grade tumors of the ovary. Cancer Res 2005;65(22):10602-12.

56. Gilks CB, Vanderhyden BC, Zhu S, van de Rijn M, Longacre TA. Distinction between serous tumors of low malignant potential and serous carcinomas based on global mRNA expression profiling. Gynecol Oncol 2005;96(3):684-94.

57. Meinhold-Heerlein I, Bauerschlag D, Hilpert F, et al. Molecular and prognostic distinction between serous ovarian carcinomas of varying grade and malignant potential. Oncogene 2005;24(6):1053-65.

58. Ouellet V, Provencher DM, Maugard CM, et al. Discrimination between serous low malignant potential and invasive epithelial ovarian tumors using molecular profiling. Oncogene 2005;24(29):4672-87.

59. Sieben NL, Oosting J, Flanagan AM, et al. Differential gene expression in ovarian tumors reveals Dusp 4 and Serpina 5 as key regulators for benign behavior of serous borderline tumors. J Clin Oncol 2005;23(29):7257-64.

60. Anglesio MS, Arnold JM, George J, et al. Mutation of ERBB2 provides a novel alternative mechanism for the ubiquitous activation of RAS-MAPK in ovarian serous low malignant potential tumors. Mol Cancer Res 2008;6(11):1678-90.

61. Dhomen N, Marais R. New insight into BRAF mutations in cancer. Curr Opin Genet Dev 2007;17(1):31-9.

62. Garnett MJ, Marais R. Guilty as charged: B-RAF is a human oncogene. Cancer Cell 2004;6(4):313-9.

63. Cuatrecasas M, Erill N, Musulen E, Costa I, Matias-Guiu X, Prat J. K-ras mutations in nonmucinous ovarian epithelial tumors: a molecular analysis and clinicopathologic study of 144 patients. Cancer 1998;82(6):1088-95.

64. Singer G, Kurman RJ, Chang HW, Cho SK, Shih le M. Diverse tumorigenic pathways in ovarian serous carcinoma. Am J Pathol 2002;160(4):1223-8.

65. Stiewe T. The p53 family in differentiation and tumorigenesis. Nat Rev Cancer 2007;7(3):165-8. 
66. O'Neill CJ, Deavers MT, Malpica A, Foster H, McCluggage WG. An immunohistochemical comparison between low-grade and high-grade ovarian serous carcinomas: significantly higher expression of p53, MIB1, BCL2, HER-2/neu, and C-KIT in high-grade neoplasms. Am J Surg Pathol 2005;29(8):1034-41.

67. Vogelstein B, Lane D, Levine AJ. Surfing the p53 network. Nature 2000;408(6810):307-10.

68. Nakayama K, Takebayashi Y, Namiki T, et al. Comprehensive allelotype study of ovarian tumors of low malignant potential: potential differences in pathways between tumors with and without genetic predisposition to invasive carcinoma. Int J Cancer 2001;94(4):605-9.

69. Soussi T. p53 alterations in human cancer: more questions than answers. Oncogene 2007;26(15):2145-56.

70. De Meyer T, Bijsmans I, Van de Vijver K, et al. E2Fs mediate a fundamental cell-cycle deregulation in high-grade serous ovarian carcinomas. J Pathol 2008;217(1):14-20.

71. Kmet LM, Cook LS, Magliocco AM. A review of p53 expression and mutation in human benign, low malignant potential, and invasive epithelial ovarian tumors. Cancer 2003;97(2):389-404.

72. Bartel F, Jung J, Bohnke A, et al. Both germ line and somatic genetics of the p53 pathway affect ovarian cancer incidence and survival. Clin Cancer Res 2008;14(1):89-96.

73. Bali A, O'Brien PM, Edwards LS, Sutherland RL, Hacker NF, Henshall SM. Cyclin D1, p53, and p21Waf1/Cip1 expression is predictive of poor clinical outcome in serous epithelial ovarian cancer. Clin Cancer Res 2004;10(15):5168-77.

74. Skomedal H, Kristensen GB, Abeler VM, Borresen-Dale AL, Trope C, Holm R. TP53 protein accumulation and gene mutation in relation to overexpression of MDM2 protein in ovarian borderline tumours and stage I carcinomas. J Pathol 1997;181(2):158-65.

75. Devlin J, Elder PA, Gabra H, Steel CM, Knowles MA. High frequency of chromosome 9 deletion in ovarian cancer: evidence for three tumour-suppressor loci. $\mathrm{Br} J$ Cancer 1996;73(4):420-3.

76. Nowee ME, Snijders AM, Rockx DA, et al. DNA profiling of primary serous ovarian and fallopian tube carcinomas with array comparative genomic hybridization and multiplex ligation-dependent probe amplification. J Pathol 2007;213(1):46-55.

77. Teodoridis JM, Hall J, Marsh S, et al. CpG island methylation of DNA damage response genes in advanced ovarian cancer. Cancer Res 2005;65(19):8961-7.

78. Esteller M, Cordon-Cardo C, Corn PG, et al. p14ARF silencing by promoter hypermethylation mediates abnormal intracellular localization of MDM2. Cancer Res 2001;61(7):2816-21.

79. Makarla PB, Saboorian MH, Ashfaq R, et al. Promoter hypermethylation profile of ovarian epithelial neoplasms. Clin Cancer Res 2005;11(15):5365-9.

80. Burkhart DL, Sage J. Cellular mechanisms of tumour suppression by the retinoblastoma gene. Nat Rev Cancer 2008;8(9):671-82.

81. Gras E, Pons C, Machin P, Matias-Guiu X, Prat J. Loss of heterozygosity at the RB-1 locus and pRB immunostaining in epithelial ovarian tumors: a molecular, immunohistochemical, and clinicopathologic study. Int J Gynecol Pathol 2001;20(4):335-40.

82. Farley J, Smith LM, Darcy KM, et al. Cyclin E expression is a significant predictor of survival in advanced, suboptimally debulked ovarian epithelial cancers: a Gynecologic Oncology Group study. Cancer Res 2003;63(6):1235-41.

83. Rosenberg E, Demopoulos RI, Zeleniuch-Jacquotte A, et al. Expression of cell cycle regulators p57(KIP2), cyclin D1, and cyclin E in epithelial ovarian tumors and survival. Human pathology 2001;32(8):808-13.

84. Marone M, Scambia G, Giannitelli C, et al. Analysis of cyclin E and CDK2 in ovarian cancer: gene amplification and RNA overexpression. Int J Cancer 1998;75(1):34-9. 
85. Etemadmoghadam D, deFazio A, Beroukhim R, et al. Integrated genome-wide DNA copy number and expression analysis identifies distinct mechanisms of primary chemoresistance in ovarian carcinomas. Clin Cancer Res 2009;15(4):1417-27.

86. Reimer D, Sadr S, Wiedemair A, et al. Clinical relevance of E2F family members in ovarian cancer--an evaluation in a training set of 77 patients. Clin Cancer Res 2007;13(1):144-51.

87. Hallstrom TC, Mori S, Nevins JR. An E2F1-dependent gene expression program that determines the balance between proliferation and cell death. Cancer Cell 2008;13(1):1122.

88. Lazzerini Denchi E, Helin K. E2F1 is crucial for E2F-dependent apoptosis. EMBO reports 2005;6(7):661-8.

89. Phillips AC, Ernst MK, Bates S, Rice NR, Vousden KH. E2F-1 potentiates cell death by blocking antiapoptotic signaling pathways. Molecular cell 1999;4(5):771-81.

90. Anttila MA, Kosma VM, Hongxiu J, et al. p21/WAF1 expression as related to p53, cell proliferation and prognosis in epithelial ovarian cancer. Br J Cancer 1999;79(11-12):1870-8.

91. Venkitaraman AR. Cancer susceptibility and the functions of BRCA1 and BRCA2. Cell 2002;108(2):171-82.

92. Narod SA, Foulkes WD. BRCA1 and BRCA2: 1994 and beyond. Nat Rev Cancer 2004;4(9):665-76.

93. Tan DS, Rothermundt C, Thomas K, et al. "BRCAness" Syndrome in Ovarian Cancer: A CaseControl Study Describing the Clinical Features and Outcome of Patients With Epithelial Ovarian Cancer Associated With BRCA1 and BRCA2 Mutations. J Clin Oncol 2008; 26(34):5530-6

94. Turner N, Tutt A, Ashworth A. Hallmarks of 'BRCAness' in sporadic cancers. Nat Rev Cancer 2004;4(10):814-9.

95. Risch HA, McLaughlin JR, Cole DE, et al. Prevalence and penetrance of germline BRCA1 and BRCA2 mutations in a population series of 649 women with ovarian cancer. Am J Hum Genet 2001;68(3):700-10.

96. Engelman JA, Luo J, Cantley LC. The evolution of phosphatidylinositol 3-kinases as regulators of growth and metabolism. Nature reviews 2006;7(8):606-19.

97. Shayesteh L, Lu Y, Kuo WL, et al. PIK3CA is implicated as an oncogene in ovarian cancer. Nat Genet 1999;21(1):99-102.

98. Yuan TL, Cantley LC. PI3K pathway alterations in cancer: variations on a theme. Oncogene 2008;27(41):5497-510.

99. Cantley LC, Neel BG. New insights into tumor suppression: PTEN suppresses tumor formation by restraining the phosphoinositide 3-kinase/AKT pathway. Proc Natl Acad Sci U S A 1999;96(8):4240-5.

100. Yin Y, Shen WH. PTEN: a new guardian of the genome. Oncogene 2008;27(41):5443-53.

101. Zhang L, Yang N, Katsaros D, et al. The oncogene phosphatidylinositol 3'-kinase catalytic subunit alpha promotes angiogenesis via vascular endothelial growth factor in ovarian carcinoma. Cancer Res 2003;63(14):4225-31.

102. Mayr D, Kanitz V, Anderegg B, et al. Analysis of gene amplification and prognostic markers in ovarian cancer using comparative genomic hybridization for microarrays and immunohistochemical analysis for tissue microarrays. American journal of clinical pathology 2006;126(1):101-9.

103. Nakayama K, Nakayama N, Jinawath $\mathrm{N}$, et al. Amplicon profiles in ovarian serous carcinomas. Int J Cancer 2007;120(12):2613-7.

104. Campbell IG, Russell SE, Choong DY, et al. Mutation of the PIK3CA gene in ovarian and breast cancer. Cancer Res 2004;64(21):7678-81.

105. Nakayama K, Nakayama N, Kurman RJ, et al. Sequence mutations and amplification of PIK3CA and AKT2 genes in purified ovarian serous neoplasms. Cancer Biol Ther 2006;5(7):779-85. 
106. Wang Y, Helland A, Holm R, Kristensen GB, Borresen-Dale AL. PIK3CA mutations in advanced ovarian carcinomas. Hum Mutat 2005;25(3):322.

107. Kurose K, Zhou XP, Araki T, Cannistra SA, Maher ER, Eng C. Frequent loss of PTEN expression is linked to elevated phosphorylated Akt levels, but not associated with p27 and cyclin D1 expression, in primary epithelial ovarian carcinomas. Am J Pathol 2001;158(6):2097-106.

108. Tashiro $\mathrm{H}$, Blazes MS, $\mathrm{Wu}$, et al. Mutations in PTEN are frequent in endometrial carcinoma but rare in other common gynecological malignancies. Cancer Res 1997;57(18):3935-40.

109. Lassus $\mathrm{H}$, Sihto $\mathrm{H}$, Leminen $\mathrm{A}$, et al. Gene amplification, mutation, and protein expression of EGFR and mutations of ERBB2 in serous ovarian carcinoma. J Mol Med 2006;84(8):671-81.

110. Lee $\mathrm{CH}$, Huntsman DG, Cheang MC, et al. Assessment of Her-1, Her-2, And Her-3 expression and Her-2 amplification in advanced stage ovarian carcinoma. Int J Gynecol Pathol 2005;24(2):147-52.

111. Schilder RJ, Sill MW, Chen X, et al. Phase II study of gefitinib in patients with relapsed or persistent ovarian or primary peritoneal carcinoma and evaluation of epidermal growth factor receptor mutations and immunohistochemical expression: a Gynecologic Oncology Group Study. Clin Cancer Res 2005;11(15):5539-48.

112. Astanehe A, Arenillas D, Wasserman WW, et al. Mechanisms underlying $p 53$ regulation of PIK3CA transcription in ovarian surface epithelium and in ovarian cancer. J Cell Sci 2008;121(Pt 5):664-74.

113. Sabbatini P, McCormick F. Phosphoinositide 3-OH kinase (PI3K) and PKB/Akt delay the onset of p53-mediated, transcriptionally dependent apoptosis. The Journal of biological chemistry 1999;274(34):24263-9. 


\section{CHAPTER 2}

\section{Loss of SerpinA5 protein expression is associated with advanced-stage serous ovarian tumors}

Ingrid T.G.W. Bijsmans, Kim M. Smits, Pauline de Graeff, G. Bea A. Wisman, Ate G.J. van der Zee, Brigitte F. Slangen, Adriaan P. de Bruïne, Manon van Engeland, Nathalie L.G. Sieben, and Koen K. Van de Vijver

Mod. Pathol. 2011; 24 (3): 463-70 


\begin{abstract}
Epithelial ovarian cancer, the most lethal neoplasm of the female genital tract, is usually diagnosed at an advanced stage since obvious symptoms are absent at early stages. This disease is believed to originate from malignant transformation of the ovarian surface epithelium or fallopian tube. Histologically, several subtypes are being recognized, with serous histology accounting for the majority of cases. Serous tumors include serous borderline tumors (SBTs) and serous carcinomas (SCAs). Better understanding of the tumor biology and molecular mechanisms involved in these tumors is needed, since both patient management and prognosis differ substantially. Previous microarray analysis identified SerpinA5, an uPA-inhibitor, as key regulator for indolent borderline behavior. Since SCAs are characterized by loss of SerpinA5 mRNA expression, we hypothesized that SerpinA5 protein expression is reduced or lost in SCAs as compared to SBTs. We performed SerpinA5 immunohistochemical staining on 32 SBTs, 187 primary SCAs and 62 omental serous metastases. Reduced or absent SerpinA5 protein staining was observed in SCAs as compared to SBTs $(p<0.001)$. SerpinA5 protein expression was significantly lowered in the omental metastases $(p<0.001)$ when compared to the matching primary SCA. Interestingly, SerpinA5 protein expression was reduced in advanced-stage SBTs, often characterized by micropapillary growth and/or microinvasion, when compared to early-stage SBTs $(p=0.015)$. In conclusion, SerpinA5 expression is significantly reduced in advancedstage SBTs and SCAs when compared to the early-stage counterparts, and reduction of expression is linked to more aggressive features of SBTs.
\end{abstract}




\section{Introduction}

Surface epithelial stromal tumors of the ovary are a leading cause of death among women suffering from cancer of the female genital tract. This disease is usually diagnosed at an advanced stage since obvious symptoms are absent at early stages. Worldwide, ovarian cancer was estimated at 225,000 cases and 140,000 deaths in 2008 [1]. Epithelial ovarian cancer is believed to be caused by malignant transformation of the ovarian surface epithelium [2]. However, current evidence suggests that high-grade serous carcinomas (HG-SCAs) might originate from the fallopian tube [3-5]. Serous borderline tumors (SBTs) belong to an intermediate group of ovarian tumors, characterized by arborizing papillae lined by stratified epithelial cells, varying atypia and lack of destructive stromal invasion. Thirty percent of SBTpatients present with tumor deposits, so-called implants, on peritoneal surfaces, omentum and in lymph nodes. However, the 5 -year survival remains excellent $(>90 \%)$ $[2,6,7]$. In contrast, SCAs show obvious stromal invasion associated with an average 5year survival of $25 \%[6,8]$. The molecular mechanisms involved in SBT and SCA development are being unraveled. $B R A F$ and $K R A S$ mutations are frequently detected in SBTs $[9,10]$. This was the first clue indicating that the RAS-RAF-MEK-ERK-MAP kinase signaling pathway is important in SBT tumorigenesis. TP53 mutations are present in up to $80 \%$ of SCAs, while almost absent in SBTs $[6,11]$. Microarray expression profiling studies have confirmed that SBTs and SCAs represent different disease entities [12-16]. We identified a putative tumor suppressor gene (TSG) SerpinA5, also referred to as plasminogen activator inhibitor-3 (PAl-3) or protein $C$ inhibitor $(P C l)$, as being differentially expressed between SBTs and SCAs [16]. SerpinA5 is a member of the serine protease inhibitor superfamily [17] which inactivates a variety of plasma proteases, including urokinase-type plasminogen activator (UPA), anticoagulant serine proteases, blood coagulation factors and fibrinolytic enzymes [18]. The presence of SerpinA5 in SBTs indicates that despite active RAS signaling, activation of downstream genes involved in extracellular matrix degradation is absent, while loss of SerpinA5 mRNA expression in SCAs was associated with downstream activation of matrix metalloproteinase 9 (MMP9) [16], explaining the invasive potential of SCAs due to degradation of the extracellular matrix. Additional lines of evidence indicate that SerpinA5 is associated with invasiveness. SerpinA5 expression is decreased in renal, prostate and ovarian cancer [16, 19, 20]. Overexpression of SerpinA5 results in decreased invasion, metastatic potential and angiogenesis in breast cancer [21] and elevated levels of SerpinA5 protein was reported to be associated with a better survival in primary breast carcinomas [22]. The aim of the current study was to investigate the relation between SerpinA5 protein expression and histopathological characteristics in SBTs and SCAs by immunohistochemistry. 


\section{Materials and Methods}

\section{Patients and pathologic review}

Formalin-fixed paraffin-embedded tissue of 76 serous tumors was obtained from the archives of the Departments of Pathology, Maastricht University Medical Center (MUMC; Maastricht, The Netherlands) and Stichting PAMM (Eindhoven, The Netherlands). Tissue microarrays of 177 primary SCAs and 88 omental metastases were obtained from the Department of Gynecologic Oncology, University Medical Center Groningen (UMCG; Groningen, The Netherlands) [23]. Review of haematoxylin and eosin slides was performed for consensus diagnosis by two experienced gynecologic pathologists (NS, KVdV). Serous tumors were considered invasive (=SCA) if destructive stromal invasion was present. SCAs were divided in low-grade (grade 1; SCA1) and high-grade (grade 2; SCA2, and grade 3; SCA3). Micropapillary growth pattern was characterized by a filigree pattern of highly complex micropapillae growing non-hierarchically. In SBTs, microinvasion was defined as the presence of single cells or nests of cells in the stroma [6]. The MUMC series consisted of 32 SBTs (3 with microinvasion, 7 with micropapillary growth and 1 with both microinvasion and micropapillary growth) and 44 SCAs (5 SCA1s, 14 SCA2s, and 25 SCA3s). None of the patients received chemotherapy prior to surgery. See Table 1 for detailed clinicopathological characteristics and Figure 1 for the characteristics of the populations.

Table 1: Patient characteristics

\begin{tabular}{|c|c|c|c|c|}
\hline Characteristics & & $\begin{array}{l}\text { MUMC } \\
(n=76)\end{array}$ & $\begin{array}{l}\text { UMCG } \\
\text { primary SCA } \\
(n=143)^{*}\end{array}$ & $\begin{array}{l}\text { UMCG } \\
\text { matched primary SCA } \\
\text { and omental metastasis } \\
(n=62)^{*}\end{array}$ \\
\hline & & $\mathrm{n}(\%)$ & $n(\%)$ & $\mathrm{n}(\%)$ \\
\hline & SBT & $32(42.1)$ & & \\
\hline & SCA1 & $5(6.6)$ & $20(14.0)$ & $3(4.8)$ \\
\hline & SCA2 & $14(18.4)$ & $43(30.1)$ & $18(29.0)$ \\
\hline & SCA3 & $25(32.9)$ & $80(55.9)$ & $41(66.1)$ \\
\hline \multicolumn{5}{|l|}{ Age at diagnosis } \\
\hline \multirow[t]{4}{*}{ Years (range) } & SBT & $49.9(19-89)$ & & \\
\hline & SCA1 & $53.0(26-75)$ & $52.7(21-78)$ & $66.7(60-78)$ \\
\hline & SCA2 & $56.8(37-80)$ & $59.9(22-84)$ & $60.1(22-84)$ \\
\hline & SCA3 & $65.5(43-79)$ & $57.6(36-84)$ & $55.7(36-81)$ \\
\hline \multicolumn{5}{|l|}{ FIGO stage\# } \\
\hline \multirow[t]{3}{*}{ SBT } & stage I & $22(71.0)$ & & \\
\hline & stage II & $1(3.2)$ & & \\
\hline & stage III & $8(25.8)$ & & \\
\hline \multirow[t]{4}{*}{ SCA } & stage I & $6(13.6)$ & $10(7.0)$ & 0 \\
\hline & stage II & $2(4.5)$ & $12(8.5)$ & 0 \\
\hline & stage III & $33(75.0)$ & $98(69.0)$ & $51(82.3)$ \\
\hline & stage IV & $3(6.8)$ & $22(15.5)$ & $11(17.7)$ \\
\hline
\end{tabular}

${ }^{*}$ In this table, only those patients of which SerpinA5 immunohistochemical staining was successful are included \#FIGO stage was unavailable for 1 SBT and 1 SCA 


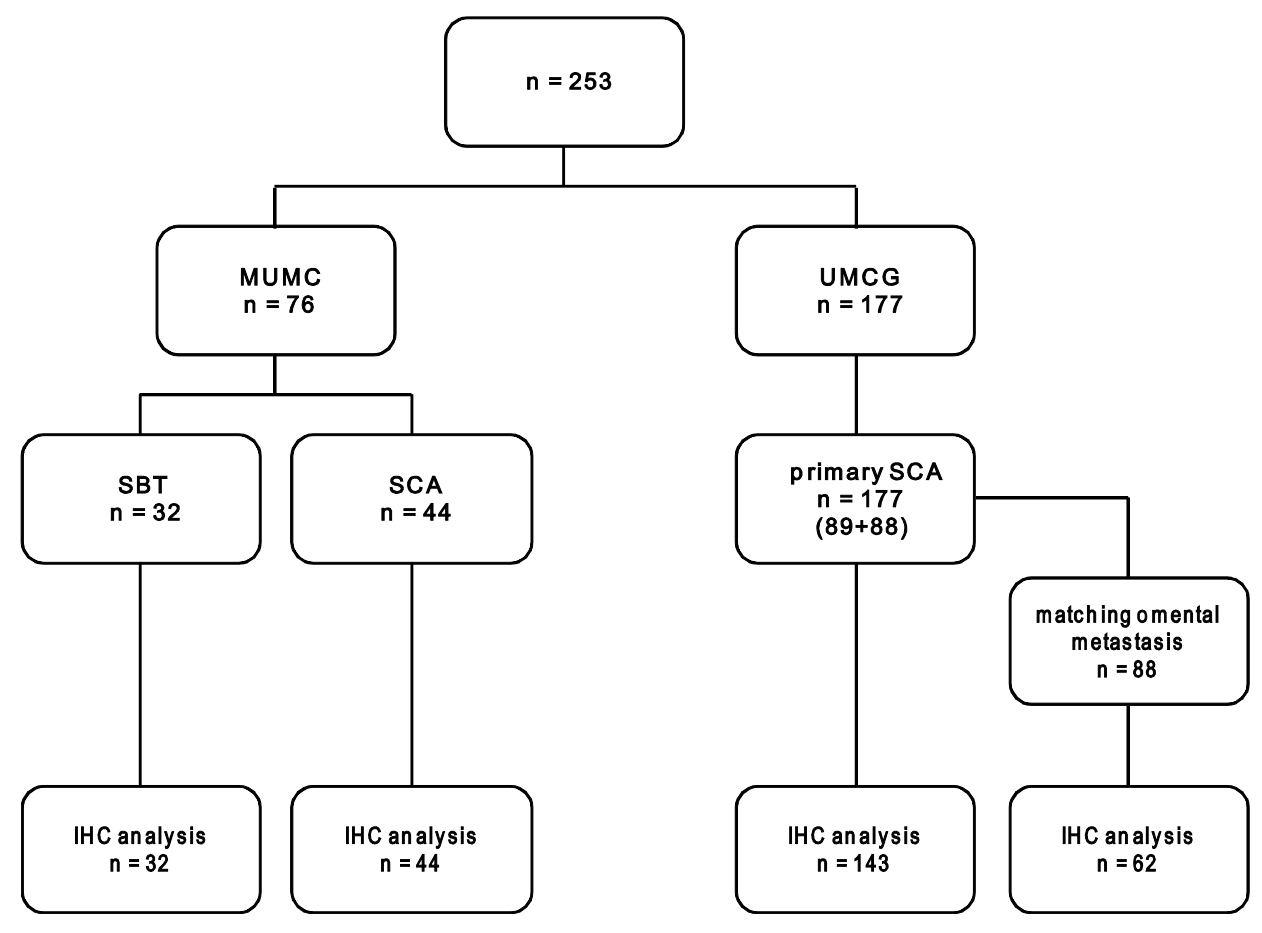

Figure 1: Patient populations and SerpinA5 IHC staining

Flowchart patient populations and SerpinA5 immunohistochemical analysis, representing the number of samples of which SerpinA5 immunohistochemical staining was successful. Patients were excluded if less than two tissue cores contained tumor cells or more than two cores were non-evaluable due to core loss during the staining process. MUMC: Maastricht University Medical Center; UMCG: University Medical Center Groningen; IHC: immunohistochemical staining

Collection, storage and use of tissue and patient data were performed in agreement with the 'Code for Proper Secondary Use of Human Tissue in The Netherlands' (http://www.fmwv.nl). This study was approved by the Medical Ethical Committee of the MUMC, Maastricht, The Netherlands.

Statistically significant differences between the SCAs derived from MUMC and UMCG were not identified for the baseline characteristics tumor grade, FIGO (International Federation of Gynecology and Obstetrics) stage, unilateral vs. bilateral tumors, and age at diagnosis (data not shown). Therefore, these were considered as one population.

At the end of this study, 36 patients (24 SBTs, 12 SCAs) of the MUMC series were alive. Patients who died within five years after diagnosis and of which autopsy was not performed, were considered 'dead of disease' if no other cause of death was specified. 27 patients (1 SBT and 26 SCAs) died of disease, 3 (2 SBTs and 1 SCA) died of unrelated causes, and 10 (5 SBTs and 5 SCAs) were lost to follow-up. From the 177 UMCG cases, 71 patients were alive (28 with evidence of disease and 43 without), 105 died (101 cancer related and 4 unrelated), and outcome of 1 patient was missing. 
SerpinA5 immunohistochemical staining and slide evaluation

Rabbit anti-human SerpinA5 polyclonal antibody was obtained from Proteintech Group, Inc (Bioconnect, Huissen, The Netherlands). One representative formalin-fixed paraffin-embedded tumor block was selected for immunohistochemical staining (IHC). Tissue sections were depararaffinized and incubated for $15 \mathrm{~min}$ in $0.6 \%$ hydrogen peroxide-methanol to block endogenous peroxidase. Antigen retrieval was performed in Tris-EDTA buffer, pH 9.0 (Klinipath, Duiven, The Netherlands) in a microwave-oven, followed by cooling in buffer for $30 \mathrm{~min}$. Sections were blocked with $5 \%$ BSA for 30 min. Slides were incubated with primary antibody (1:250) for $60 \mathrm{~min}$, followed by 30 min incubation with poly-HRP-anti-mouse/rabbit/rat IgG (Immunologic, Duiven, The Netherlands). Liquid DAB (Dako, Carpinteria, USA) was applied for $15 \mathrm{~min}$. Slides were counterstained with haematoxylin and mounted. As a negative control, primary antibody was omitted. Evaluation of full and tissue microarray sections was performed by two observers (IB and KVdV) without prior knowledge of clinicopathological characteristics. Discrepant scores were re-evaluated to reach consensus. Cytoplasmic staining of epithelial cells was considered positive. For scoring tumor sections spotted on TMAs, at least two out of four cores for each tumor had to be available; otherwise the sample was scored as "not available". If tumor cells were not present in more than two cores, a sample was scored as "not determined". Slides lacking protein expression were scored as negative. The percentage of cells showing SerpinA5 protein expression was graded $<10 \%$ (range: zero to focal staining), $11-40 \%$ (up to $40 \%$ positive stained tumor cells), or $>40 \%$. Staining of blood vessels was used as an internal positive control. If heterogeneity in staining pattern within one tumor section was observed, the average score of this section was determined. Mean staining intensity of samples spotted on TMAs was taken from 2 to 4 core biopsies.

\section{Data analysis}

Differences between baseline characteristics, protein expression in SBTs and SCAs, primary tumors and omental metastases, and SCAs from MUMC and UMCG, were determined by Pearson Chi-Square Test and T-test where appropriate (SPSS 15.0 software). Cancer-specific survival was defined as the time from diagnosis until cancerrelated death or end of follow-up. Kaplan-Meier curves and log-rank tests were used to estimate the influence of SerpinA5 expression on cause-specific survival. Hazard ratios (HR) and corresponding 95\% confidence intervals (Cl) were assessed by Cox proportional hazard analyses adjusted for age at diagnosis, tumor grade, FIGO stage and tumor limited to one or both ovaries, which are possible confounders known as prognostic factors for ovarian cancer and possibly influencing the crude HR. Analysis was also performed after stratification for age. The proportional hazard assumption was tested using the Schoenfeld residuals and the log $(-\log )$ hazard plots. Survival analyses were done using STATA 15.0. All $p$-values are two-sided and $p \leq 0.05$ were considered statistically significant. 


\section{Results}

SerpinA5 protein expression was decreased in SCAs as compared to SBTs $(p<0.001)$ (Table 2). This is in concordance with previous results showing decreased mRNA expression [16]. SBTs expressed SerpinA5 protein in all cases, with the majority of cases $(22 / 32 ; 68.8 \%)$ showing $>40 \%$ staining. This is in contrast to SCAs which showed $<10 \%$ expression (defined as no or focal protein expression) for most cases (28/44; 63.6\%). Comparing SerpinA5 protein expression between SBTs and LG-SCAs, as well as between SBTs and HG-SCAs, showed a statistically significant reduction $(p<0.001)$ in both groups of SCAs. A representative SerpinA5 staining is shown in Figure 2.

Table 2: SerpinA5 protein expression in SBTs and SCAs

\begin{tabular}{|c|c|c|c|c|c|c|}
\hline \multirow[b]{2}{*}{ Hospital } & \multirow[b]{2}{*}{ Tumor (n) } & & & \multicolumn{3}{|c|}{$\begin{array}{c}\text { Staining score } \\
\mathrm{n}(\%)\end{array}$} \\
\hline & & & & $<10 \%$ & $11-40 \%$ & $>40 \%$ \\
\hline \multirow[t]{10}{*}{ MUMC } & SBT (32) & & & $3(9.4)$ & $7(21.9)$ & $22(68.8)$ \\
\hline & & type & typical (21) & $1(4.8)$ & $4(19.0)$ & $16(76.2)$ \\
\hline & & & MP and/or MI* (11) & $2(18.2)$ & $3(27.3)$ & $6(54.5)^{\prime}$ \\
\hline & & FIGO\# & early (23) & $1(4.3)$ & $3(13.0)$ & $19(82.6)$ \\
\hline & & & advanced (8) & $2(25.0)$ & $4(50.0)$ & $2(25.0)$ \\
\hline & SCA (44) & & & $28(63.6)$ & $13(29.5)$ & $3(6.8)$ \\
\hline & & grade & LG-SCA (5) & $2(40.0)$ & $2(40.0)$ & $1(20.0)$ \\
\hline & & & HG-SCA (39) & $26(66.7)$ & $11(28.2)$ & $2(5.1)$ \\
\hline & & FIGO & early (8) & $6(75.0)$ & $1(12.5)^{\prime}$ & $1(12.5)$ \\
\hline & & & advanced (36) & $22(61.1)$ & $12(33.3)$ & $2(5.6)$ \\
\hline \multirow[t]{7}{*}{ UMCG } & SCA (143) & & & $81(56.6)$ & $40(28.0)$ & $22(15.4)$ \\
\hline & & grade & LG-SCA (20) & $7(35.0)$ & $9(45.0)$ & $4(20.0)^{\prime}$ \\
\hline & & & HG-SCA (123) & $74(60.2)$ & $31(25.2)$ & $18(14.6)$ \\
\hline & & FIGO\# & early (22) & $10(45.5)$ & $7(31.8)^{\prime}$ & $5(22.7)^{\prime}$ \\
\hline & & & advanced (120) & $71(59.2)$ & $32(26.7)$ & $17(14.2)$ \\
\hline & paired (62) & & SCA & $35(56.5)$ & $21(33.9)$ & $6(9.7)$ \\
\hline & & & omental tissue & $45(72.6)$ & $13(21.0)$ & $4(6.5)$ \\
\hline
\end{tabular}

*MP: SBT with micropapillary growth; MI: SBT with microinvasion

\#FIGO stage was unavailable for 1 SBT with MP and 1 SCA

The UMCG series was used as a second, independent series for validation of data obtained from the MUMC series. Successful SerpinA5 staining results were obtained in $143 / 177(80.8 \%)$ of SCAs. Thirty-four SCAs were excluded from analysis because less than two tissue cores contained tumor cells $(n=10)$, or more than two cores were nonevaluable due to core loss during the staining process $(n=24)$. SerpinA5 protein expression in SCAs (Table 2) was not statistically different $(p=0.340)$ between the MUMC and UMCG series: Table 2 shows that 28/44 (63.6\%) SCAs of the MUMC group show less than $10 \%$ expression, which is in concordance with the $81 / 143$ (56.6\%) SCAs of the UMCG group, thereby confirming loss or decrease of SerpinA5 protein expression in SCAs. 

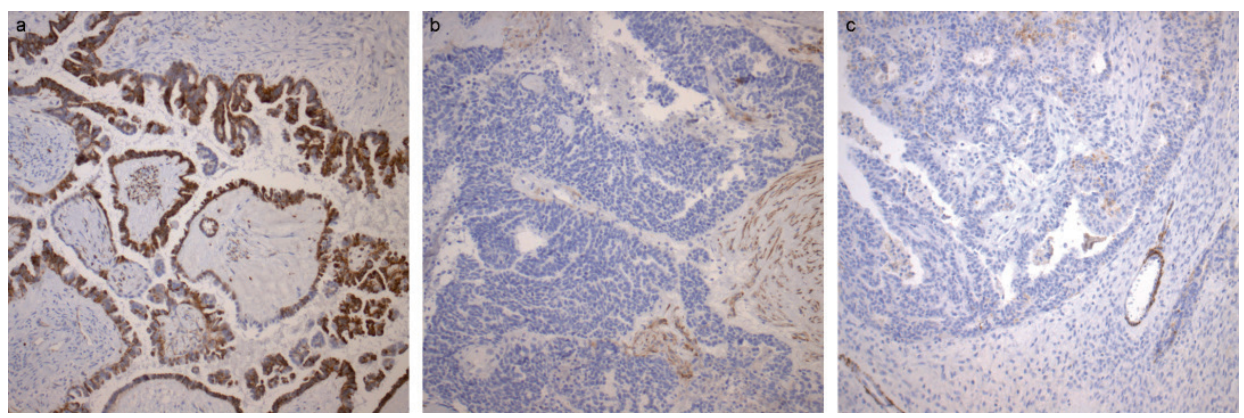

\section{Figure 2: SerpinA5 protein expression in SBT and SCAs}

Representative immunohistochemical staining of $>40 \%$ SerpinA5 expression in SBT (a), no expression (b) or focal $(<10 \%)$ (c) SerpinA5 expression in SCA. This panel also shows SerpinA5 expression in blood vessels, used as internal positive control.

No difference was seen in SerpinA5 protein expression when stratifying the SCAs for FIGO stage, but when comparing the 25 LG-SCAs with the 162 HG-SCAs, we detected a statistically significant higher SerpinA5 protein expression in the LG-SCAs $(p=0.038)$. This difference in LG-SCAs and HG-SCAs was also detected when the analysis was performed in advanced-stage (FIGO stage III/IV) patients only $(p=0.002)$, whereas SerpinA5 protein expression in early-stage (FIGO stage I/II) patients is similar $(p=0.539)$. In SBTs, a statistically significant decrease $(p=0.015)$ in SerpinA5 protein expression was observed in advanced vs. early-stage. In stage I SBT-patients $18 / 22$ (81.8\%) cases showed $>40 \%$ SerpinA5 expression. Stage III SBT-patients showed $>40 \%$ SerpinA5 protein expression in $2 / 8(25.0 \%)$ cases. The reduced SerpinA5 expression level in LG-SCAs as compared to SBTs remained statistically different when stratified for early FIGO stage $(p<0.001)$, whereas no difference was detected between advanced-stage SBT and advanced-stage LG-SCA $(p=0.845)$. The primary SCA and matching omental metastasis were available for 88 cases. SerpinA5 staining was successful in $62 / 88(70.5 \%)$ of paired cases. Statistical analysis showed that SerpinA5 protein expression was significantly $(p<0.001)$ higher in primary SCAs as compared to the corresponding metastases (Table 2; Figure 3). Metastatic tissues showed SerpinA5 protein levels $<10 \%$ in $45 / 62$ ( $72.6 \%)$, whereas this was $35 / 62$ (56.5\%) of primary SCAs.
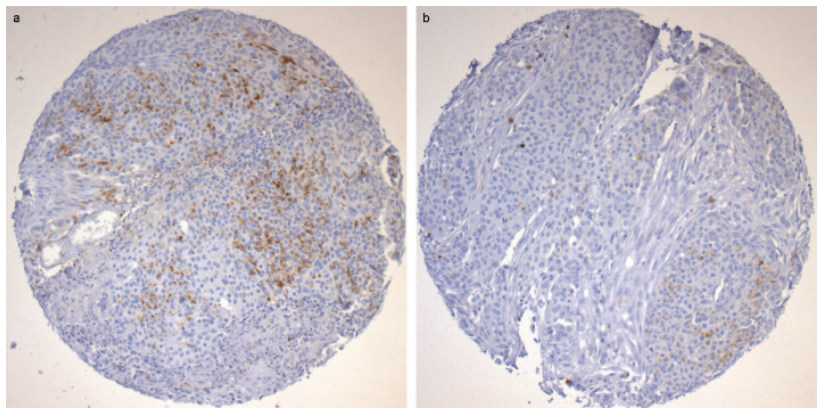

Figure 3: SerpinA5 protein expression is decreased in metastases as compared to primary SCA SerpinA5 protein expression in a representative primary SCA (a) and its matching omental metastasis (b). 
Kaplan-Meier analysis showed no statistical significant difference in cancer-specific survival in patients with low $(<10 \%)$, moderate $(11-40 \%)$ or high $(>40 \%)$ SerpinA5 protein expression in SBTs (log-rank; $p=0.649$ ) or SCAs ( $p=0.525$; MUMC and UMCG together). Multivariate Cox regression analysis showed that SerpinA5 protein expression was not associated with cause-specific survival, whereas age at diagnosis, FIGO stage and tumor grade were associated with decreased survival.

\section{Discussion}

This study supports other publications $[19,21]$ that a decrease in SerpinA5 protein expression is associated with invasion. We hypothesized that SerpinA5 protein expression is decreased or lost in SCAs as compared to SBTs based on mRNA expression results [16]. Here we show significantly higher SerpinA5 protein expression in SBTs as compared to SCAs $(p<0.001)$. An interesting finding was that SerpinA5 expression was decreased in advanced-stage SBTs as compared to early-stage SBTs. Examination of H\&E slides showed that 6 of the 8 advanced-stage SBTs demonstrated microinvasion and/or micropapillary architecture, features of more aggressive SBTs. Of these 6 patients, only 1 patient had a SerpinA5 protein expression of $>40 \%$. In earlystage SBTs microinvasion was not present, a micropapillary growth pattern was only seen in 4 of 23 cases, however with SerpinA5 protein expression of $>40 \%$ in all four cases. Decrease or loss of SerpinA5 protein expression might contribute to this microinvasive and/or micropapillary phenotype. Performing a staging procedure in SBT cases with lowered SerpinA5 protein expression might thus be recommended. Comparing SBTs and LG-SCAs for SerpinA5 protein expression showed a statistically significant reduction in early-stage LG-SCAs vs. early-stage SBTs, whereas advancedstage SBTs did not differ from advanced-stage LG-SCAs. These data indicate that advanced-stage SBTs are more similar and probably closely related to advanced-stage LG-SCAs. A drawback of the present study is that the groups are small (8 SBTs and 18 LG-SCAs with advanced-stage disease). Therefore, independent, larger SBT and LG-SCA populations are needed to confirm these results. SerpinA5 protein expression differed significantly between LG-SCAs and HG-SCAs, and although subgroups are small, and data thus have to be interpreted with caution, these data point toward the current literature on LG-SCAs and HG-SCAs that LG-SCAs are more similar to SBTs than to HGSCAs [24]. SCAs are defined as invasive based on morphology on H\&E slides, whereas SBTs lack destructive stromal invasion. Loss of SerpinA5 protein expression might be associated with the invasive properties of SCAs. Extracellular matrix (ECM) degradation is facilitated by UPA, providing tumor cells to invade surrounding tissue. SerpinA5 is an UPA-inhibitor, preventing the conversion of plasminogen to plasmin and subsequent ECM degradation [25]. Expression of uPA and downstream activation of MMP9 occurs in SCAs that have lost or decreased SerpinA5 protein expression, which is followed by degradation of the ECM. We and others have shown increased MMP9 levels in SCAs as compared to SBTs $[16,26]$. Schmalfeldt et al. showed the highest pro-MMP9 levels in metastases [26]. Here we show that SerpinA5 protein expression was significantly lower in metastatic tissue as compared to the primary tumor. Loss of SerpinA5 
expression can contribute to increased MMP activity and subsequent invasive and metastatic potential.

Two main pathways involved in serous ovarian cancer include the RAS signaling pathway and the TP53 pathway. The RAS signaling pathway is constitutively active in approximately $60 \%$ of SBTs $[9,10]$. We hypothesized that activation of this pathway is associated with SerpinA5 expression to prevent downstream ECM degradation [16]. However, BRAF or KRAS mutations were not associated with SerpinA5 protein expression in SBTs (data not shown). A feedback loop between TP53 and UPA expression has been demonstrated in lung epithelial cells [27, 28]. Reintroduction of TP53 in TP53-deficient lung epithelial cells suppressed uPA expression. PAI-1, a family member of SerpinA5 was shown to be minimally expressed in TP53-deficient lung carcinoma cells [28]. In SCAs, TP53 mutations occur in up to $80 \%$ of cases. It is reasonable that SerpinA5 is downregulated in SCAs as a result of this mutation. TP53 mutational status was available for SCAs derived from MUMC. 24 of the $44(58.5 \%)$ SCAs contained a TP53 mutation. A statistically significant difference in SerpinA5 protein expression in relation to TP53 mutation was not found (data not shown). Although loss of SerpinA5 protein expression seems to be associated with invasiveness, it is not a prognostic factor as survival of SCA patients was unaffected. These results were also seen for SerpinA1 and TIMP-1 in ovarian cancer [29, 30]. These data indicate that other "hallmarks" of cancer [31] in addition to invasion are presumably involved in determining patient outcome.

In conclusion, we showed that SerpinA5 protein expression decreases in advanced stages, invasive serous tumors and the corresponding metastases. Loss of SerpinA5 is associated with micropapillary growth and microinvasion in SBTs and the invasive phenotype of SCAs.

\section{Acknowledgements}

We thank Thom Boerman from Stichting PAMM, Eindhoven, The Netherlands, for providing material of SBT cases. 


\section{References}

1. Ferlay J, Shin HR, Bray F, Forman D, Mathers C, Parkin DM. Estimates of worldwide burden of cancer in 2008: GLOBOCAN 2008. Int J Cancer 2010;127(12):2918-27.

2. Hart WR. Borderline epithelial tumors of the ovary. Mod Pathol 2005;18 Suppl 2:S33-50.

3. Crum CP, Drapkin R, Miron A, et al. The distal fallopian tube: a new model for pelvic serous carcinogenesis. Curr Opin Obstet Gynecol 2007;19(1):3-9.

4. Kurman RJ, Shih le M. The origin and pathogenesis of epithelial ovarian cancer: a proposed unifying theory. The American journal of surgical pathology;34(3):433-43.

5. Marquez RT, Baggerly KA, Patterson AP, et al. Patterns of gene expression in different histotypes of epithelial ovarian cancer correlate with those in normal fallopian tube, endometrium, and colon. Clin Cancer Res 2005;11(17):6116-26.

6. Prat J. Surface epithelial-stromal tumors: Serous tumors: Saunders; 2004: 83-111

7. Scully R.E. Young, R.H., Clement P.B. Surface epithelial-stromal tumours, serous tumors. Atlas of Tumor Pathology; Tumors of the Ovary, Maldeveloped Gonads, Fallopian Tube, and Broad Ligament: Armed Forces Institute of Pathology; Washington, DC, 1998; 1996.

8. Cannistra SA. Cancer of the ovary. N Engl J Med 2004;351(24):2519-29.

9. Sieben NL, Macropoulos P, Roemen GM, et al. In ovarian neoplasms, BRAF, but not KRAS, mutations are restricted to low-grade serous tumours. J Pathol 2004;202(3):336-40.

10. Singer $G$, Oldt $R$, 3rd, Cohen $Y$, et al. Mutations in BRAF and KRAS characterize the development of low-grade ovarian serous carcinoma. J Natl Cancer Inst 2003;95(6):484-6.

11. Shih le M, Kurman RJ. Molecular pathogenesis of ovarian borderline tumors: new insights and old challenges. Clin Cancer Res 2005;11(20):7273-9.

12. Bonome T, Lee JY, Park DC, et al. Expression profiling of serous low malignant potential, low-grade, and high-grade tumors of the ovary. Cancer Res 2005;65(22):10602-12.

13. Gilks CB, Vanderhyden BC, Zhu S, van de Rijn M, Longacre TA. Distinction between serous tumors of low malignant potential and serous carcinomas based on global mRNA expression profiling. Gynecol Oncol 2005;96(3):684-94.

14. Meinhold-Heerlein I, Bauerschlag D, Hilpert F, et al. Molecular and prognostic distinction between serous ovarian carcinomas of varying grade and malignant potential. Oncogene 2005;24(6):1053-65.

15. Ouellet V, Provencher DM, Maugard CM, et al. Discrimination between serous low malignant potential and invasive epithelial ovarian tumors using molecular profiling. Oncogene 2005;24(29):4672-87.

16. Sieben NL, Oosting J, Flanagan AM, et al. Differential gene expression in ovarian tumors reveals Dusp 4 and Serpina 5 as key regulators for benign behavior of serous borderline tumors. J Clin Oncol 2005;23(29):7257-64.

17. Huntington JA, Li W. Structural insights into the multiple functions of protein $\mathrm{C}$ inhibitor. Cell Mol Life Sci 2009; 66(1):113-21.

18. Geiger M. Protein C inhibitor, a serpin with functions in- and outside vascular biology. Thromb Haemost 2007;97(3):343-7.

19. Wakita T, Hayashi T, Nishioka J, et al. Regulation of carcinoma cell invasion by protein C inhibitor whose expression is decreased in renal cell carcinoma. Int J Cancer 2004;108(4):516-23.

20. Cao $\mathrm{Y}$, Becker $\mathrm{C}$, Lundwall $\mathrm{A}$, et al. Expression of protein $\mathrm{C}$ inhibitor $(\mathrm{PCl})$ in benign and malignant prostatic tissues. Prostate 2003;57(3):196-204.

21. Asanuma K, Yoshikawa T, Hayashi $T$, et al. Protein $C$ inhibitor inhibits breast cancer cell growth, metastasis and angiogenesis independently of its protease inhibitory activity. Int J Cancer 2007;121(5):955-65. 
22. Castello R, Landete JM, Espana F, et al. Expression of plasminogen activator inhibitors type 1 and type 3 and urokinase plasminogen activator protein and mRNA in breast cancer. Thromb Res 2007;120(5):753-62.

23. de Graeff $\mathrm{P}$, Hall J, Crijns AP, et al. Factors influencing $\mathrm{p} 53$ expression in ovarian cancer as a biomarker of clinical outcome in multicentre studies. British journal of cancer 2006;95(5):627-33.

24. Kurman RJ, Shih le M. Pathogenesis of ovarian cancer: lessons from morphology and molecular biology and their clinical implications. Int J Gynecol Pathol 2008;27(2):151-60.

25. Smith HW, Marshall CJ. Regulation of cell signalling by uPAR. Nature reviews;11(1):23-36.

26. Schmalfeldt $B$, Prechtel $D$, Harting $K$, et al. Increased expression of matrix metalloproteinases (MMP)-2, MMP-9, and the urokinase-type plasminogen activator is associated with progression from benign to advanced ovarian cancer. Clin Cancer Res 2001;7(8):2396-404.

27. Shetty S, Gyetko MR, Mazar AP. Induction of $p 53$ by urokinase in lung epithelial cells. The Journal of biological chemistry 2005;280(30):28133-41.

28. Shetty S, Padijnayayveetil J, Tucker T, Stankowska D, Idell S. The fibrinolytic system and the regulation of lung epithelial cell proteolysis, signaling, and cellular viability. American journal of physiology 2008;295(6):L967-75.

29. Normandin K, Peant B, Le Page C, et al. Protease inhibitor SERPINA1 expression in epithelial ovarian cancer. Clinical \& experimental metastasis;27(1):55-69.

30. Steffensen KD, Waldstrom M, Christensen RK, Bartels A, Brunner N, Jakobsen A. Lack of relationship between TIMP-1 tumour cell immunoreactivity, treatment efficacy and prognosis in patients with advanced epithelial ovarian cancer. BMC cancer;10:185.

31. Hanahan D, Weinberg RA. The hallmarks of cancer. Cell 2000;100(1):57-70. 


\section{CHAPTER 3}

\section{E2Fs mediate a fundamental cell cycle deregulation in high-grade serous ovarian carcinomas}

Ingrid T.G.W. Bijsmans*, Tim De Meyer*, Koen K. Van de Vijver, Sofie Bekaert, Jan Oosting, Wim Van Criekinge, Manon van Engeland, and Nathalie L.G. Sieben

* These authors contributed equally to this work

J Pathol. 2009; 217 (1): 14-20 


\begin{abstract}
Several studies described a role for the $E 2 F / R b$ pathway in ovarian serous carcinomas (SCAs). Since $E 2 F / R b$ pathway deregulation is a general hallmark of human cancer, it remains unclear whether this deregulation is of particular importance in SCAs or whether it reflects a common oncologic feature. Here, we have clarified this issue by the examination of microarray expression profiles of SCAs and particularly by the comparison with another, less malignant, ovarian cancer type, serous borderline tumors (SBTs). Results were validated by quantitative RT-PCR, both on the microarray samples and on an independent panel, and TP53 mutation analysis was performed. This integrated analysis revealed a significant increase in the expression of the transcription factors E2F1 and E2F3 in SCAs, when compared to SBTs. This was associated with vast overexpression of E2F target genes in SCAs compared to SBTs. High-grade SCAs (HG-SCAs) in particular exhibited a major deregulated E2F target expression pattern. Generally, overexpression of E2F targets in SCAs appeared to be well structured since those targets considered negative regulators of the cell cycle or promoters of apoptosis were usually not overexpressed in SCAs. Similar to E2F target deregulation, TP53 mutations were identified in HG-SCA, to a lesser extent in lowgrade SCA (LG-SCA), and not in SBTs. These results suggest that a structured, generally upregulated $E 2 F$ transcription factor activity is associated with a global cell-cycle disturbance in HG-SCAs and exceeds typical E2F/Rb pathway disruption in tumors, at least compared with SBTs.
\end{abstract}




\section{Introduction}

In contrast to its rather low incidence (the average lifetime risk for women is about 1 out of 70), ovarian cancer represents the most lethal neoplasm of the female genital tract $[1,2]$. The large majority of carcinomas are serous (SCAs) and in addition to the long-held belief in an ovarian epithelial origin [3], current evidence suggests that highgrade SCAs (HG-SCAs) might arise from the distal fallopian tube [4-6]. Serous borderline tumors (SBTs) are characterized by multiple fibrous papillae, covered by crowded epithelial cells forming multilayer cellular tufts, and mild-to-moderate nuclear atypia [7,8]. Although $30 \%$ of SBT patients present with tumor deposits in the peritoneal cavity, so-called implants, the 10-year survival in general remains excellent. From a clinical point of view, this is inconsistent with metastatic disease, leading to the classification 'borderline' [9]. It has been proposed that SBTs are SCA precursors [10]. However, recently we and others have identified activating mutations in BRAF or KRAS, two members of the RAS-RAF-MEK-ERK-MAP kinase pathway, in about $60 \%$ of SBTs, in contrast to less than $12 \%$ KRAS mutations and no BRAF mutations in SCAs $[11,12]$. Large-scale activation of genes further downstream of the MAPK pathway in SBTs is, however, absent, probably due to the activity of genes such as DUSP4 and SerpinA5, which inhibit activation of MMP9 [13]. Together with the observation that SCAs cluster separately from SBTs in unsupervised hierarchical clustering of expression data, these results suggest that most SCAs are unrelated to SBTs [13]. A clue for the origin of HGSCAs comes from the high frequency of TP53 mutations (up to 80\%), which are less common in low-grade SCAs (LG-SCAs) and rare in SBTs [14-16]. One way by which TP53 exerts its tumor suppressive activity is through modulation of the $E 2 F / R b$ pathway (Figure 1). E2Fs are transcription factors involved in cell growth inhibition and apoptosis, but, on the other hand, also in cell cycle progression and tumor growth. Examples of E2F targets are TP53 and E2F1 themselves, indicating complex feedback mechanisms. The importance of the $E 2 F / R b$ pathway in carcinogenesis is now considered a general hallmark of cancer $[17,18]$. $E 2 F / R b$ pathway disruption reporting differential expression of E2Fs and/or E2F target genes has been described [19-21]. While this might reflect the general disruption in tumors, E2Fs and their targets have also been associated with platinum resistance and even survival in SCA patients $[20,22]$. Consequently, the question arises as to whether the impact in SCAs might exceed the typical deregulation in tumors and even play a central role in SCA carcinogenesis. Therefore, microarray expression profiles of SCAs were investigated and compared with SBT profiles. Unlike healthy controls, SBTs should reflect the basic $E 2 F$ deregulation in tumors and thus provide a better reference set for the current analysis. If there were no difference in $E 2 F / R b$ deregulation between SBTs and SCAs, despite the important clinical and biological differences, this would indicate that the observed $E 2 F / R b$ deregulation in SCAs simply reflects a phenomenon seen in all tumors and that it is unlikely to play a central role in SCA tumor biology. 


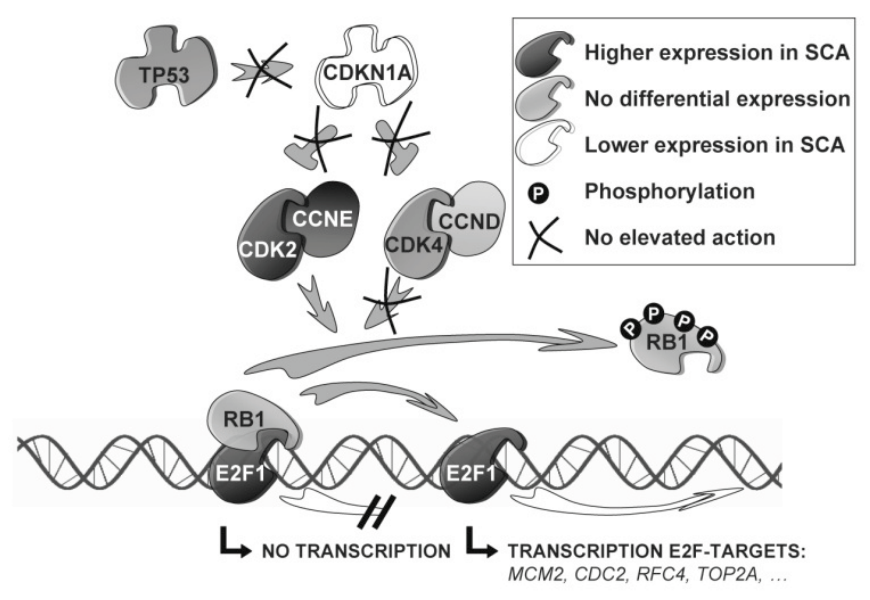

Figure 1: E2F1/Rb pathway

Simplified overview of the $E 2 F 1 / R b$ pathway applied to ovarian cancer. Lower and higher expression in SCAs is relative to SBTs.

\section{Materials and methods}

\section{Tissue samples}

The procedures followed to obtain patient samples and to perform RNA isolation, cRNA synthesis, and Affymetrix Focus microarray (Santa Clara, CA, USA) gene expression profiling have been described previously [13]. In brief, the expression levels of 13 SBTs (including two micropapillary SBTs), 10 SCAs grade 1 (SCA1s; LG-SCAs) and 15 SCAs grade 3 (SCA3s; HG-SCAs) samples were analyzed on the microarray. Nine technical replicates were included. The raw data have been submitted to the Gene Expression Omnibus (BSE12471). After normalization, the technical replicates clustered immediately adjacent to each other [13]. Normalized expression values of technical replicates were averaged for further analysis. In addition, a second, independent panel consisting of fresh frozen tissue of 6 SBTs and 17 SCAs (3 SCA1s, and 14 SCA3s) was collected from the archive of the Department of Pathology, University Hospital Maastricht. Total RNA was isolated using the GenElute ${ }^{\mathrm{TM}}$ Mammalian Total RNA Miniprep Kit (Sigma-Aldrich, Zwijndrecht, The Netherlands) according to the manufacturer's instructions. None of the patients received chemotherapy prior to tumor harvesting (detailed clinicopathological characteristics are summarized in Supplementary Table S1). This study was approved by the local institutional review boards.

\section{Data analysis}

A detailed description of the data analysis, principally performed in $\mathrm{R}$ version 2.7.0, is provided in the Supplementary Methods. In summary, the AffyExpress package was used to perform microarray quality control; significance analysis of microarrays (SAM), to select differentially expressed genes [23]; and the global test, to test if differences in 
the expression of a group of genes are associated with a certain clinical outcome. An initial pathway exploration was performed using Ingenuity Pathway Analysis software (Ingenuity ${ }^{\circledR}$ Systems, Redwood City, CA, USA).

\section{Quantitative RT-PCR}

RT-PCR was carried out for CCNE1, E2F1, E2F3, and CDKN1A. We made use of the MyIQ ${ }^{\text {TM }}$ Single-Color Real-Time PCR Detection System (Biorad). Expression of the genes was normalized to the expression of the housekeeping gene $\beta$-actin (for details see Supplementary Methods and Supplementary Table S2).

\section{TP53 mutation analysis}

For all samples, exons 5-8 of TP53, spanning the DNA binding domain, were analyzed for mutations. Details and primer sequences are listed in Supplementary Methods and Supplementary Table S2.

\section{Results}

Microarray quality control and significance analysis of microarrays (SAM)

The microarray quality characteristics are available in Supplementary Figure S1. SAM resulted in a subset of 312 differentially expressed probes, corresponding to 304 unique genes (details are available in Supplementary Tables S3 and S4 and Figure S2). In this set, 139 genes were associated with higher expression in SCAs, while 165 showed higher expression in SBTs.

\section{Ingenuity Pathway Analysis confirms E2F involvement}

Ingenuity Pathway Analysis software was used for preliminary identification of differentially expressed pathways. Two major networks were detected, both involved in cell-cycle regulation, revealing central roles for E2F1 and CDKN1A (p21). Both networks showed a considerable overlap and were therefore merged in Supplementary Figure S3. This figure also highlights the importance of E2F3, another $E 2 F$ family member. This not only confirms the significance of $E 2 F$ transcription factors, but even suggests a central role for the $E 2 F / R b$ pathway in the difference between SBT and SCA tumor biology.

\section{Analysis of E2F target genes in SCAs and SBTS}

In order to assess the importance of a deregulated $E 2 F$ transcription factor activity in SCAs, we analyzed the presumed differential expression of the E2F target genes. Therefore, target genes were compiled from data published by Bracken et al., who summarized a large number of E2F target genes [17], as well as from Bieda et al., who performed a high-throughput E2F1 ChIP-on-Chip study [24]. The gene list derived from Bracken et al. contained 118 genes present on our HG-Focus microarray. From the publicly available data of Bieda et al., we selected those $E 2 F$ targets that belonged to the top 2000 of the median ranked results in both replicates of the repeated experiment (details of the experiment are described in ref [24]). This is well within the cut-offs proposed by Bieda et al., thereby avoiding false-positive results, and resulted 
in 304 unique genes represented on the HG-Focus microarray. Taking both lists together, 46 genes overlapped, resulting in a final list of 376 unique target genes (Figure 2).

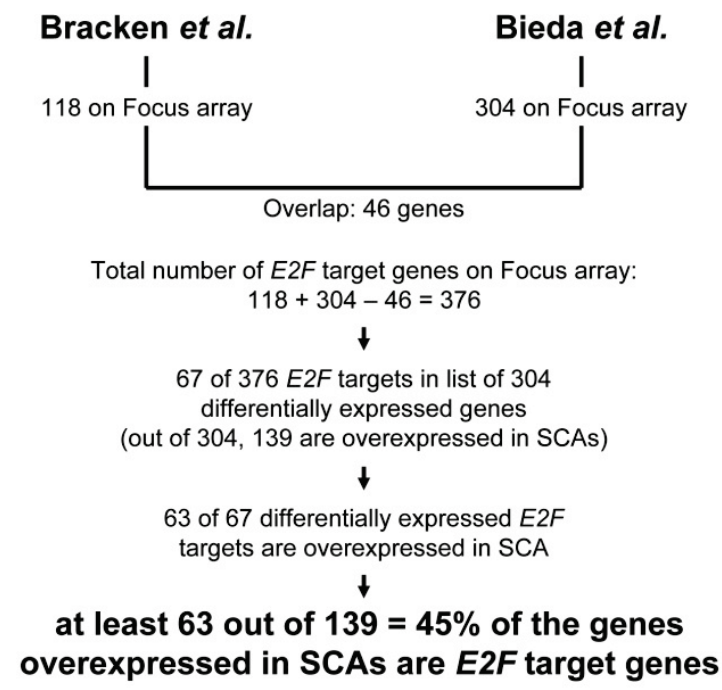

Figure 2: E2F target compilation

Schematic overview of the compilation of E2F targets and comparison with genes differentially expressed between SCAs and SBTs.

Global test analysis revealed that these E2F target genes, as a whole, were clearly differentially expressed $\left(p=4 \times 10^{-6}\right)$. Gene-wise, 67 E2F targets belonged to the list of 304 differentially expressed genes. Strikingly, only four of these targets showed lower expression in SCAs, while 63 genes were overexpressed in SCAs. This implies that of all the genes designated as overexpressed in SCA, at least $45 \%$ are E2F targets (Figure 2). $E 2 F$ pathway deregulation is clearly a major feature of SCAs. This is illustrated in Figure 3 , which depicts the complete linkage hierarchical clustering of SBTs and SCAs based solely on all E2F targets present on the microarray. Two major clusters were retrieved which largely correspond to the two tumor types: one cluster consisting of only SCAs and one cluster consisting of all SBTs and four SCA1s.

\section{Analysis of E2F target genes in SCA1s and SCA3s}

In order to allow a more detailed analysis, the SCAs were divided according to their grade into SCA1s and SCA3s. This resulted in a full separation between SBTs and SCA3s, while in SCA1s, several E2F target expression patterns clearly more resembled SBTs (Figure 3). The latter is probably associated with the borderline significant difference in $E 2 F$ target expression levels between SCA1s and SCA3s (global test, $p=0.05$ ). Since this indicates that there might be a substantial difference between SCA1s and SCA3s, E2F target expression patterns of the two grades were individually compared with SBTs. The global test demonstrated that SCA3s in particular exhibited a different $E 2 F$ target expression pattern from SBTs $\left(p=2.5 \times 10^{-6}\right)$. This was also the case for SCA1s; however, 
to a lesser extent $(p=0.001)$. This is probably caused by the fact that the $E 2 F$ target expression patterns of several SCA1s were more similar to SBTs (Figure 3).

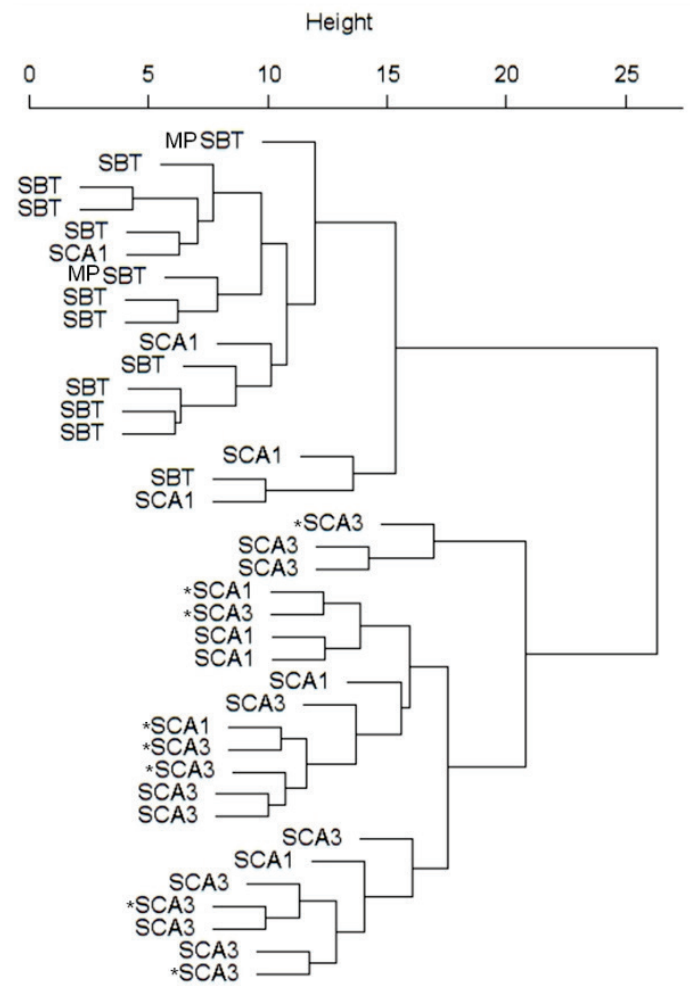

Figure 3: Hierarchical clustering of SBTs, SCA1s, and SCA3s based on E2F targets

Complete hierarchical clustering analysis based on the total set of $E 2 F$ targets reveals a complete separation between SBTs and SCA3s. Several SCA1s appear in the SBT cluster.

MPSBT: micropapillary serous borderline tumor

*Samples with TP53 mutation in exons 5-8.

Genes differentially expressed between SBTs and respectively SCA1s and SCA3s were identified with SAM. To be comparable with previous results (SBTs vs. SCAs), again 312 probes were designated as differentially expressed for each analysis. Again, most differentially expressed E2F targets were upregulated, compared with SBTs. In SCA3s, 66 of the 69 differentially expressed E2F targets were upregulated. SCA1s showed upregulation of only 17 out of 27 differentially expressed $E 2 F$ target probes. However, 15 out of the 17 upregulated E2F targets in SCA1s were also upregulated in SCA3s, suggesting a similar $E 2 F / R b$ pathway deregulation in SCA3s and in at least a large proportion of SCA1s (Supplementary Table S5). These results demonstrate that SCA3s and an important fraction of SCA1s are characterized by a similarly deregulated $E 2 F / R b$ pathway. Therefore, differences in tumor grades were no longer considered in further E2F target analysis. 


\section{Quantitative $R T-P C R$ validation}

CCNE1, an E2F target that was not represented on the HG-Focus microarray, plays a crucial role in the activation of E2F1-induced G1-S cell-cycle progression (Figure 1) [17]. Therefore, in addition to the validation of several key role-playing genes, i.e. $E 2 F 1, E 2 F 3$, and $C D K N 1 A$, we chose to assess the potential differential expression of CCNE1. Quantitative RT-PCR (Figure 4) was performed on those microarray samples for which a sufficient RNA quantity was available (10 SBTs and 24 SCAs) and on an independent sample set of 6 SBTs and 17 SCAs. In the case of E2F3, sufficient RNA quantities were available only for 8 SBTs from the microarray set, providing a possible explanation for the non-significant result there. E2F3 was, however, differentially expressed in the independent set, and the other genes in both sets. Altogether, these results validate the most important microarray results and demonstrate higher CCNE1 expression in SCAs than in SBTs.
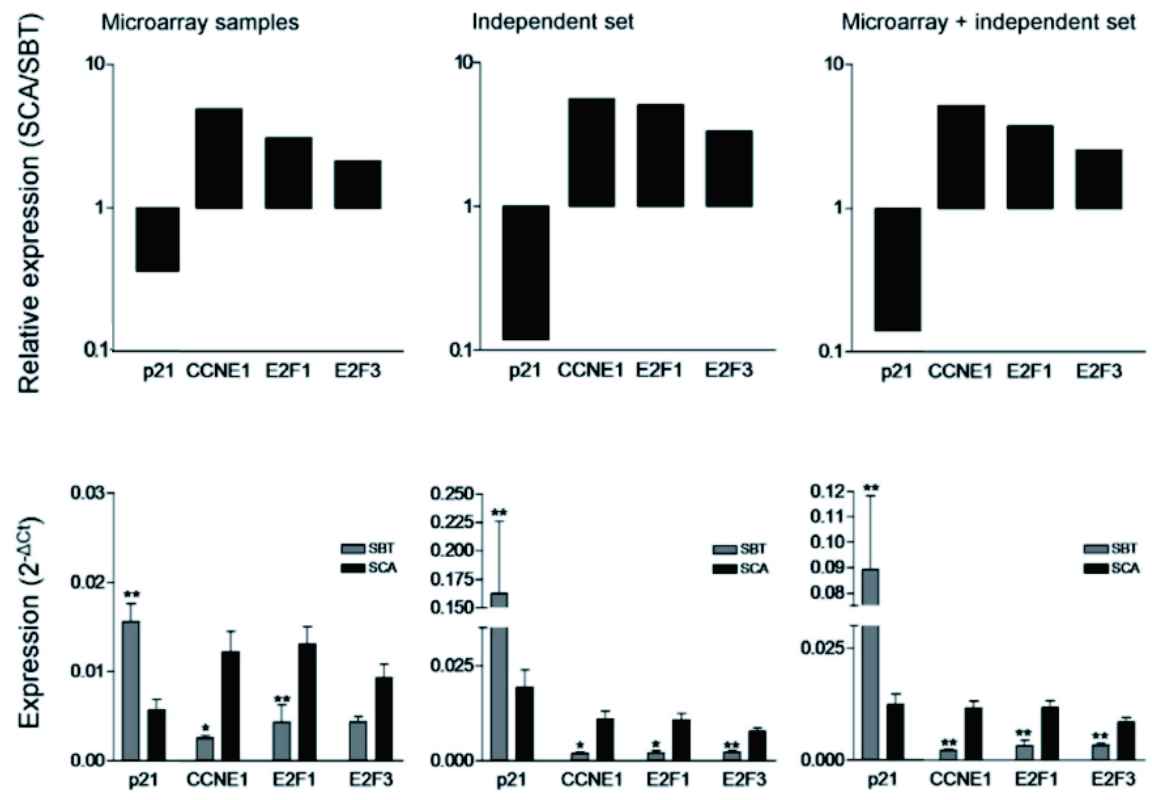

Figure 4: Quantitative RT-PCR validation

RT-PCR results of CDKN1A (p21), CCNE1, E2F1, and E2F3 on microarray samples, an independent panel of samples, and both panels together. The upper panel represents relative expression. Absolute expression levels are depicted in the lower panel. ${ }^{*} p<0.01$ and ${ }^{* *} p<0.001$.

\section{E2F target upregulation in SCAs depends on gene function}

Since the different functions of E2F targets are largely divergent, the question arises as to whether the general upregulation of these targets also depends on their gene functions. Table 1 summarizes a number of biological processes in which $E 2 F$ targets are involved, according to Bracken et al. [17], and the number of targets differentially expressed for each biological process. The target genes involved, and the 
corresponding biological processes, are also indicated in Supplementary Table S4. The chi-square test reveals that the differentially expressed targets are not randomly distributed throughout the different biological processes $(p<0.001)$. Indeed, $E 2 F / R b$ pathway upregulation appears to be restricted to tumor growth-promoting processes, e.g. cell-cycle and DNA synthesis, while apoptosis and negative cell cycle regulation targets are generally not differentially expressed (Table 1).

Table 1: Differentially expressed E2F targets according to biological process

\begin{tabular}{ll}
\hline Biological process $^{*}$ & Upregulated E2F targets\# \\
\hline Cell cycle & $17 / 28$ \\
- G1 & $0 / 4$ \\
- G1/S & $5 / 10$ \\
- S/G2 & $12 / 14$ \\
DNA damage repair & $5 / 13$ \\
Development & $1 / 13$ \\
DNA synthesis and replication & $13 / 31$ \\
Negative regulators of cell cycle & $0 / 5$ \\
Checkpoints & $6 / 10$ \\
Apoptosis & $0 / 12$ \\
Differentiation & $0 / 6$ \\
\hline
\end{tabular}

${ }^{*}$ Classified according to Bracken et al. [17]

\#Number of E2F targets upregulated/number of E2F targets on microarray

The corresponding genes have been indicated in Supplementary Table S4

\section{TP53 mutation analysis}

We analyzed all SCAs and SBTs for TP53 mutations. An overview of this analysis is shown in Supplementary Table S6. Microarray samples characterized by mutations in exons 5-8 are indicated in Figure 3. While no mutations were found in the 19 SBTs analyzed, 16/37 SCAs (43\%) exhibited mutations in the analyzed exons (Fisher exact test, $p<0.001$ ). Within SCAs, mutations were found in 3/12 SCA1s and 13/25 SCA3s (insufficient amount of DNA or no result for 1 SCA1 and 4 SCA3s), a difference that was not significant (Fisher exact test, $\mathrm{p}=0.166$ ).

\section{Discussion}

The scope of this study was to validate and assess the impact of $E 2 F / R b$ pathway deregulation in SCAs. Since disruption of the $E 2 F / R b$ pathway is a common feature of virtually all human tumors [18], microarray profiles were compared with low malignant potential serous ovarian tumors, also called SBTs. We were able to detect that a vast amount, at least $45 \%$ in our data set, of the genes that are overexpressed in SCAs (compared with SBTs) were E2F targets. E2F target expression patterns of both SCA1s and SCA3s were significantly different from those in SBTs. E2F target based clustering, however, revealed that several SCA1s appeared to be more similar to SBTs than to SCA3s. On the other hand, some SCA1s also exhibited TP53 mutations, which is typical for SCAs. These results suggest that although SCA1s appear to be heterogeneous with respect to $E 2 F$ target expression, the majority of SCAs, and particularly HG-SCAs, are characterized by a major $E 2 F / R b$ pathway deregulation. The magnitude of this deregulation is illustrated by Ingenuity Pathway Analysis, dedicating central roles to 
$E 2 F 1, E 2 F 3$, and CDKN1A in the discrimination between SCAs and SBTs. These results clearly indicate that in SCAs, the $E 2 F / R b$ deregulation surpasses standard disruption in human tumors, at least as reflected in SBTs, and should be considered a major oncologic feature in the majority of SCAs. CDKN1A was upregulated in SBTs and was identified by Ingenuity Pathway Analysis to play an important role in the discrimination between SBTs and SCAs. Its central function in E2F/Rb control (Figure 1 ) suggests that $C D K N 1 A$ in particular might prevent SBTs from ending up in a cascade of $E 2 F$ target upregulation and massive cell-cycle deregulation. Several of the involved targets have already been described. Bonome et al. also reported E2F3 and $C D C 2$ to be upregulated in late-stage high-grade carcinomas compared with normal ovarian epithelium. Higher CDKN1A expression was typical for borderline tumors [19]. Higher expression of E2F1, CCNE1, and CDK2 in SCAs, compared with SBTs, has also been reported by others $[21,25]$. Higher E2F1 protein expression in ovarian cancer and of CDKN1A in SBTs has been demonstrated using immunochemical staining [26,27]. Furthermore, most of the pathways already reported to be deregulated in SCAs [19] can be attributed to this E2F malfunctioning, e.g. S and G2-M checkpoint regulation, DNA replication, and mitosis. This comparison supports our findings and conclusions, and also fits a large part of earlier reported results in this major $E 2 F / R b$ disruption. An interesting observation was that there was barely any differential expression for certain $E 2 F$ target subgroups, including genes involved in apoptosis and negative regulation of the cell-cycle. One example of such a pro-apoptotic target is DUSP4 (MKP2) [28,29]. This protein, proposed to be partially responsible for the benign behavior of SBTs, was not overexpressed in SCAs, but is downregulated in this tumor [13]. This is to be expected to a certain degree, since even in normal cells not all E2F targets should function simultaneously. Additional regulation is therefore indispensable. Again, this supports a role for the E2F/Rb pathway beyond typical tumor background levels. An in-depth expression study, preferably using arrays with a higher coverage, might clarify the molecular mechanisms responsible for the uncoupling between the expression of different $E 2 F$ target subgroups. For example, the pro-apoptotic activity of E2F1 can function both TP53-dependent or -independent [30]. The high frequency of TP53 mutations in SCAs might therefore provide a clue for this lack of E2F1-mediated transcription of apoptotic proteins (Figure 1). Importantly, in a recent study, PI3K/AKT signaling was identified to distinguish between the proliferative and the apoptotic function of E2F1 [31]. The interpretation of this uncoupling is further complicated by the interactions between the different E2F transcription factors. While E2F1 and E2F3 were the only differentially expressed E2Fs, they are both direct targets themselves and their overexpression in SCAs could merely reflect a global E2F deregulation. The fact that also other $E 2 F$ members are important was recently proposed by Reimer et al. They showed that the absolute and relative expression of both proliferationpromoting and proliferation-inhibiting E2F transcription factors is not only involved in ovarian cancer biology, but also influences clinical outcome, and might even contribute to platinum resistance [20]. The fact that disruption of the $E 2 F / R b$ pathway might be a factor in platinum resistance has also been illustrated by Dressman et al., who demonstrated that blocking the $E 2 F / R b$ pathway resulted in an increased platinum response in ovarian cancer cell lines characterized by an E2F3 deregulation signature [22]. Our findings support the importance of these reported results, implying a 
potential clinical impact. For example, our results suggest that blocking the $E 2 F / R b$ pathway, e.g. with CDK inhibitors [32], should be considered particularly in HG-SCAs. Furthermore, as these agents diminish the expression of DNA damage-repairing E2F targets, they will increase platinum sensitivity, in line with the results of Dressman et al. [22]. The difference in E2F/Rb deregulation between SBTs and SCAs might be related to more clinical consequences, e.g. the higher proliferation rate of SCAs compared with SBTs. As the Rb-E2F complex mediates G1-arrest triggered by contact inhibition [33], this might also explain the higher invasive potential of SCAs.

Overall, we identified a fundamental difference in the E2F activity between SBTs and SCAs, suggesting a central role for this pathway in the majority of SCAs. We were able to fit a significant number of reported differentially expressed genes and cell-cycle processes into a single theory of E2F deregulation. The key message of this report is therefore that in the majority of SCAs, in particular in HG-SCAs, the E2F/Rb pathway is not simply a deregulated pathway among others, but a major cause of other pathways being deregulated. Consequently, this points future research in the direction of the origin(s) of $E 2 F / R b$ deregulation. Ultimately, the answer may lie in revealing the mechanism causing specific groups of $E 2 F$ targets to be overexpressed in contrast with other, especially cell integrity-protecting, targets. 


\section{References}

1. Cannistra SA. Cancer of the ovary. N Engl J Med 2004;351(24):2519-29.

2. Jacobs IJ, Skates SJ, MacDonald N, et al. Screening for ovarian cancer: a pilot randomised controlled trial. Lancet 1999;353(9160):1207-10.

3. Prat J. Surface epithelial-stromal tumors: Serous tumors: Saunders; 2004: 83-111

4. Kurman RJ, Shih le M. Pathogenesis of ovarian cancer: lessons from morphology and molecular biology and their clinical implications. Int J Gynecol Pathol 2008;27(2):151-60.

5. Crum CP, Drapkin R, Miron A, et al. The distal fallopian tube: a new model for pelvic serous carcinogenesis. Curr Opin Obstet Gynecol 2007;19(1):3-9.

6. Marquez RT, Baggerly KA, Patterson AP, et al. Patterns of gene expression in different histotypes of epithelial ovarian cancer correlate with those in normal fallopian tube, endometrium, and colon. Clin Cancer Res 2005;11(17):6116-26.

7. Scully R.E. Young, R.H., Clement P.B. Surface epithelial-stromal tumours, serous tumors. Atlas of Tumor Pathology; Tumors of the Ovary, Maldeveloped Gonads, Fallopian Tube, and Broad Ligament: Armed Forces Institute of Pathology; Washington, DC, 1998; 1996.

8. Hart WR. Borderline epithelial tumors of the ovary. Mod Pathol 2005;18 Suppl 2:S33-50.

9. Prat J, De Nictolis M. Serous borderline tumors of the ovary: a long-term follow-up study of 137 cases, including 18 with a micropapillary pattern and 20 with microinvasion. Am J Surg Pathol 2002;26(9):1111-28.

10. Shih le M, Kurman RJ. Ovarian tumorigenesis: a proposed model based on morphological and molecular genetic analysis. Am J Pathol 2004;164(5):1511-8.

11. Singer $\mathrm{G}$, Oldt $\mathrm{R}, 3 \mathrm{rd}$, Cohen $\mathrm{Y}$, et al. Mutations in BRAF and KRAS characterize the development of low-grade ovarian serous carcinoma. J Natl Cancer Inst 2003;95(6):484-6.

12. Sieben NL, Macropoulos $P$, Roemen GM, et al. In ovarian neoplasms, BRAF, but not KRAS, mutations are restricted to low-grade serous tumours. J Pathol 2004;202(3):336-40.

13. Sieben NL, Oosting J, Flanagan AM, et al. Differential gene expression in ovarian tumors reveals Dusp 4 and Serpina 5 as key regulators for benign behavior of serous borderline tumors. J Clin Oncol 2005;23(29):7257-64.

14. Marks JR, Davidoff AM, Kerns BJ, et al. Overexpression and mutation of p53 in epithelial ovarian cancer. Cancer Res 1991;51(11):2979-84.

15. Salani R, Kurman RJ, Giuntoli R, 2nd, et al. Assessment of TP53 mutation using purified tissue samples of ovarian serous carcinomas reveals a higher mutation rate than previously reported and does not correlate with drug resistance. Int J Gynecol Cancer 2008;18(3):48791.

16. Teneriello MG, Ebina $\mathrm{M}$, Linnoila RI, et al. p53 and Ki-ras gene mutations in epithelial ovarian neoplasms. Cancer Res 1993;53(13):3103-8.

17. Bracken AP, Ciro M, Cocito A, Helin K. E2F target genes: unraveling the biology. Trends in biochemical sciences 2004;29(8):409-17.

18. Johnson DG, Degregori J. Putting the Oncogenic and Tumor Suppressive Activities of E2F into Context. Current molecular medicine 2006;6(7):731-8.

19. Bonome T, Lee JY, Park DC, et al. Expression profiling of serous low malignant potential, low-grade, and high-grade tumors of the ovary. Cancer Res 2005;65(22):10602-12.

20. Reimer D, Sadr S, Wiedemair A, et al. Clinical relevance of E2F family members in ovarian cancer--an evaluation in a training set of 77 patients. Clin Cancer Res 2007;13(1):144-51.

21. Gilks CB, Vanderhyden BC, Zhu S, van de Rijn M, Longacre TA. Distinction between serous tumors of low malignant potential and serous carcinomas based on global mRNA expression profiling. Gynecol Oncol 2005;96(3):684-94.

22. Dressman HK, Berchuck A, Chan G, et al. An integrated genomic-based approach to individualized treatment of patients with advanced-stage ovarian cancer. J Clin Oncol 2007;25(5):517-25. 
23. Tusher VG, Tibshirani R, Chu G. Significance analysis of microarrays applied to the ionizing radiation response. Proceedings of the National Academy of Sciences of the United States of America 2001;98(9):5116-21.

24. Bieda M, Xu X, Singer MA, Green R, Farnham PJ. Unbiased location analysis of E2F1-binding sites suggests a widespread role for E2F1 in the human genome. Genome research 2006;16(5):595-605.

25. Ouellet V, Guyot MC, Le Page C, et al. Tissue array analysis of expression microarray candidates identifies markers associated with tumor grade and outcome in serous epithelial ovarian cancer. International journal of cancer 2006;119(3):599-607.

26. Lu Z, Luo RZ, Peng $\mathrm{H}$, et al. Transcriptional and posttranscriptional down-regulation of the imprinted tumor suppressor gene ARHI (DRAS3) in ovarian cancer. Clin Cancer Res 2006;12(8):2404-13.

27. Palazzo JP, Monzon F, Burke $M$, et al. Overexpression of p21WAF1/CIP1 and MDM2 characterizes serous borderline ovarian tumors. Human pathology 2000;31(6):698-704.

28. Wang J, Shen WH, Jin YJ, Brandt-Rauf PW, Yin Y. A molecular link between E2F-1 and the MAPK cascade. The Journal of biological chemistry 2007;282(25):18521-31.

29. Cheung TH, Kwan YL, Hamady M, Liu X. Unraveling transcriptional control and cisregulatory codes using the software suite GeneACT. Genome biology 2006;7(10):R97.

30. Dimova DK, Dyson NJ. The E2F transcriptional network: old acquaintances with new faces. Oncogene 2005;24(17):2810-26.

31. Hallstrom TC, Mori S, Nevins JR. An E2F1-dependent gene expression program that determines the balance between proliferation and cell death. Cancer cell 2008;13(1):11-22 .

32. Senderowicz AM. Small-molecule cyclin-dependent kinase modulators. Oncogene 2003;22(42):6609-20.

33. Zhang HS, Postigo AA, Dean DC. Active transcriptional repression by the Rb-E2F complex mediates G1 arrest triggered by p16INK4a, TGFbeta, and contact inhibition. Cell 1999;97(1):53-61.

34. Silverberg SG. Histopathologic grading of ovarian carcinoma: a review and proposal. Int J Gynecol Pathol 2000;19(1):7-15. 


\section{Supplementary methods}

\section{Data analysis in $R$}

Statistical analyses were performed using $R$ version 2.7.0. The function AffyQA (package AffyExpress, version 1.6.0) was used for microarray data quality assessment. Significance analysis of microarrays (SAM) allows robust detection of differentially expressed genes by a modified unpaired t-test with unequal variances [23]. We used the R-version of SAM in the bioconductor Siggenes package (version 1.8.0), and chose the fudge factor to be strictly positive. In a microarray data environment, testing differential expression of a group of genes is rather difficult due to the high dimensionality of the data. The Global Test (version 4.10.0 for R) however, was specifically developed to test if differences in expression of a group of genes are associated with a certain clinical outcome. The "chisq.test" function was used to perform the chi-square test, $p$-values were simulated (10000 repeats) to take into account low expected counts. The standard "fisher.exact" function was used to perform the Fisher exact test. Complete linkage hierarchical cluster analysis was performed using the "hclust" function. For the quantitative RT-PCR results, the Wilcoxon Rank-sum test was used for the evaluation of differential gene-expression.

\section{Ingenuity Pathway Analysis}

An initial pathway exploration was performed using Ingenuity Pathways Analysis software (Ingenuity ${ }^{\circledR}$ Systems, Redwood City, California, USA). The set of differentially expressed genes was uploaded into the application. Each gene identifier was mapped to its corresponding object in the Ingenuity Pathways Knowledge Base. These genes were overlaid onto a global molecular network developed from information stored in the Ingenuity Pathways Knowledge Base. Networks of these genes were then algorithmically generated based on their connectivity and graphically represented.

\section{Quantitative RT-PCR}

The iScript ${ }^{\mathrm{TM}}$ CDNA Synthesis kit (Biorad, Veenendaal, The Netherlands) was used to reverse-transcribe one microgram of RNA according to the manufacturer's instructions. RT-PCR was carried out using 30 ng CDNA using primer sets for CCNE1, E2F1, E2F3 and CDKN1A. We made use of the MyIQ ${ }^{\text {TM }}$ Single-Color Real-Time PCR Detection System (Biorad). The reaction mix contained $I^{\mathrm{TM}} \mathrm{SYBR}^{\circledR}$ Green Supermix (Biorad) and $10 \mu \mathrm{mol} / \mathrm{l}$ of both forward and reverse primers (Eurogentec, Maastricht, The Netherlands). PCR conditions: 1 cycle of $3 \mathrm{~min}$ at $95^{\circ} \mathrm{C}, 40$ cycles of $10 \mathrm{sec}$ at $95^{\circ} \mathrm{C}$ and $45 \mathrm{sec}$ at $60^{\circ} \mathrm{C}$, followed by $1 \mathrm{~min}$ at $95^{\circ} \mathrm{C}$ and $1 \mathrm{~min}$ at $60^{\circ} \mathrm{C}$. The expression of the genes was normalized to the expression of the house keeping gene $\beta$-actin. Primer sequences are listed in Table S2.

\section{TP53 mutation analysis}

TP53 mutation analysis was successful in all SBTs, and in 37 of 40 SCAs. We were unable to perform mutation analysis of two samples because DNA was unavailable. Exons 5-8 of TP53, spanning the DNA binding domain, were analyzed for mutations using a semi-nested PCR approach. See Table S2 for primer sequences. Direct sequencing of TP53 was performed using the BigDye ${ }^{\circledR}$ Terminator v1.1 Cycle 
Sequencing Kit (Applied Biosystems). Amplified products were sequenced on the $A B I$ 3730 DNA Analyzer (Applied Biosystems). Mutation detection was performed using Mutation Surveyor DNA Variant Analysis Software v3.0 (SoftGenetics LLC, USA). Included controls are the colorectal cancer cell lines $\mathrm{CaCo} 2$ ( $\mathrm{G}>\mathrm{T}$ mutation at codon 204, exon 6; Glu >stop) and HT29 (G>A mutation at codon 273, exon 8; Arg > His). Detected mutations are listed in Table S6.

\section{Supplementary results}

\section{Microarray quality control}

The quality control results depicted in Figure S1 show generally similar raw-intensities, average backgrounds, percentage of genes called present and scaling factors. One array (Figure S1, red), of which also a technical replicate was available, exhibited relatively higher raw intensities associated with a higher average background. Since the array was previously shown to cluster immediately adjacent to its technical replicate (Figure 1 in [13]), we chose to include the array in the further analysis. The hybridization controls are all called present and the $3^{\prime} / 5^{\prime}$ ratio's for the housekeeping controls (actin and GAPDH) are relatively low.

\section{Significance analysis of microarray analysis (SAM)}

We used SAM to identify a set of differentially expressed genes. The calculated fudge factor was 0.0149. In order to avoid false positives, we selected the false discovery rate for the differentially expressed gene set in such a way that the corresponding expected number of falsely called genes was estimated as low as 0.05 gene (i.e. less than one gene). Table S3 illustrates the different SAM parameters for different delta values (for more information, see [23]). These results are depicted in Figure S2A. p0 indicates the prior probability that a gene is not differentially expressed (estimated by SAM). FDR indicates the estimated false discovery rate. Figure S2B illustrates the characteristics for the chosen delta value $=2.5$. The $d(i)$ values indicate the relative difference in gene expression. More information concerning the different parameters is available in [23] and with the R-package siggenes.

\section{Ingenuity Pathway Analysis}

The results from the Ingenuity Pathway Analysis are depicted in Figure S3.

\section{Differentially expressed E2F targets}

Table S4 shows the E2F targets designated differentially expressed between SBTs and SCAs (SAM). The biological processes (for those targets summarized in [17]) are indicated.

\section{Analysis of E2F target genes in SCA1s and SCA3}

We applied SAM to identify E2F target genes differentially expressed between respectively SCA1s and SBTs, and SCA3s and SBTs. In order to be comparable with previous results, the 312 most clearly differentially expressed probes were selected.

a) E2F targets differentially expressed between SCA1s and SBTs 
Of the 312 selected probes (corresponding with delta $=1.336$, expected number falsely called genes $=8.99$, FDR $=0.014$, fudge factor $=0.0151), 27$ were $E 2 F$ targets. While the majority (17/27) was upregulated in SCA1s, a significant amount of E2F targets (10/27) was downregulated. Up- and downregulated targets are summarized in Table S5.

b) E2F targets differentially expressed between SCA3s and SBTs

Among the 312 differentially expressed probes (corresponding with delta $=2.583$, expected number falsely called genes $=0.58$, FDR $=0.001$, fudge factor $=0.0177$ ), 67 probes corresponding to 66 unique genes, were upregulated E2F targets. Only 3 differentially expressed E2F targets were downregulated in SCA3s compared to SBTs (see Table S5).

Interestingly, there is a clear overlap between the differentially expressed E2F targets in SCA1s and SCA3s. The 3 targets with a lower expression in SCA3s (relative to SBTs) were also found as lower expressed targets in SCA1s and 15/17 upregulated E2F targets in SCA1s were also upregulated in SCA3s compared to SBTs. This suggests that the $E 2 F / R b$ deregulation in (a fraction of) SCA1s and SCA3s is similar.

\section{TP53 mutation analysis}

The results of the mutation analysis for the different samples are summarized in Table s6.

\section{Supplementary tables}

Table S1: Patient characteristics

\begin{tabular}{|c|c|c|}
\hline Characteristics & & Patients $(n=61)$ \\
\hline & $\begin{array}{l}\text { SBT } \\
\text { SCA1* } \\
\text { SCA3* }^{*}\end{array}$ & $\begin{array}{l}\mathrm{n}(\%) \\
19(31.1) \\
13(21.3) \\
29(47.5)\end{array}$ \\
\hline $\begin{array}{l}\text { Age at diagnosis } \\
\text { Years(range) }\end{array}$ & $\begin{array}{l}\text { SBT } \\
\text { SCA1 } \\
\text { SCA3 }\end{array}$ & $\begin{array}{l}48(19-74) \\
56.8(21-87) \\
62.6(43-85)\end{array}$ \\
\hline FIGO stage & $\begin{array}{l}\text { stage I } \\
\text { stage II } \\
\text { stage III } \\
\text { stage IV }\end{array}$ & $\begin{array}{l}\text { n (\%) } \\
15(25.4) \\
5(8.5) \\
37(62.7) \\
2(3.4)\end{array}$ \\
\hline Mitotic rate (per 2 & $\begin{array}{l}\text { SCA1 } \\
\text { SCA3 }\end{array}$ & $\begin{array}{l}10.7(1-32) \\
39.6(10-109)\end{array}$ \\
\hline
\end{tabular}

*Histopathologic grading according to Silverberg [34]

\#Age and FIGO stage were unavailable for 2 patients 
E2F deregulation in serous ovarian carcinomas

Insert Supplementary table 2 
E2F deregulation in serous ovarian carcinomas

Table S3: Significance analysis of microarrays (SAM); SAM-characteristics for different values of delta

\begin{tabular}{lllll}
\hline Delta & p0 & FALSE & Called & FDR \\
\hline 1 & 0.52 & 244.08 & 2253 & 0.056374 \\
1.1 & 0.52 & 150.96 & 1959 & 0.040099 \\
1.2 & 0.52 & 95.28 & 1744 & 0.028429 \\
1.3 & 58.44 & 1529 & 0.019889 \\
1.4 & 0.52 & 35.18 & 1331 & 0.013754 \\
1.5 & 0.52 & 21.08 & 1178 & 0.009312 \\
1.6 & 0.52 & 12.72 & 1027 & 0.006445 \\
1.7 & 0.52 & 7.38 & 875 & 0.004389 \\
1.8 & 0.52 & 4.32 & 788 & 0.002853 \\
1.9 & 0.52 & 2.61 & 702 & 0.001935 \\
2 & 0.52 & 1.58 & 625 & 0.001315 \\
2.1 & 0.52 & 0.79 & 511 & 0.000804 \\
2.2 & 0.52 & 0.35 & 451 & 0.000404 \\
2.3 & 0.52 & 0.26 & 413 & 0.000328 \\
2.4 & 0.52 & 0.12 & 368 & 0.00017 \\
2.5 & 0.52 & 0.05 & 312 & $8.34 \mathrm{E}-05$ \\
2.6 & 0.52 & 0.04 & 276 & $7.54 \mathrm{E}-05$ \\
2.7 & 0.52 & 0.03 & 255 & $6.12 \mathrm{E}-05$ \\
2.8 & 0.52 & 0.01 & 228 & $2.28 \mathrm{E}-05$ \\
2.9 & 0.52 & 0.01 & 203 & $2.56 \mathrm{E}-05$ \\
3 & 0.52 & 0 & 163 & 0 \\
\hline
\end{tabular}

Table S4: E2F targets differentially expressed between SBTs and SCAs

\begin{tabular}{|c|c|c|c|c|}
\hline Probe ID & UniGene ID & Symbol & Fold Change* $^{*}$ & Biological Process\# \\
\hline 209464_at & Hs.442658 & $A \cup R K B$ & 1.920705476 & Cell cycle - S/G2 \\
\hline 205345_at & Hs.591642 & BARD1 & 1.951867445 & DNA damage repair \\
\hline 205733_at & Hs.169348 & $B L M$ & 1.768648767 & \\
\hline 214727_at & Hs.34012 & BRCA2 & 1.30765895 & Checkpoints \\
\hline 209642_at & Hs.469649 & BUB1 & 2.340922889 & Checkpoints \\
\hline 203755_at & Hs.631699 & BUB1B & 2.769551015 & Checkpoints \\
\hline 219004_s_at & Hs.190518 & C21orf45 & 1.52878476 & \\
\hline 203418_at & Hs.58974 & CCNA2 & 2.316327628 & Cell cycle - S/G2 \\
\hline 213226_at & Hs.58974 & CCNA2 & 1.571250615 & Cell cycle - S/G2 \\
\hline 203213_at & Hs.334562 & $C D C 2$ & 2.362315803 & Cell cycle - S/G2 \\
\hline 202870_s_at & Hs.524947 & CDC20 & 3.416629382 & Cell cycle - S/G2 \\
\hline 204695_at & Hs.437705 & CDC25A & 1.87627374 & Cell cycle - G1/S \\
\hline 204126_s_at & Hs. 474217 & CDC45L & 1.665971091 & DNA synthesis and replication \\
\hline 203968_s_at & Hs.405958 & CDC6 & 1.68462237 & DNA synthesis and replication \\
\hline 204510_at & Hs. 533573 & $C D C 7$ & 1.744981986 & DNA synthesis and replication \\
\hline 204252_at & Hs.19192 & CDK2 & 1.518929792 & Cell cycle - G1/S \\
\hline 204962_s_at & Hs.1594 & CENPA & 3.558639557 & \\
\hline 205046_at & Hs.75573 & CENPE & 1.689463776 & Checkpoints \\
\hline 200021_at & Hs. 170622 & CFL1 & 1.419213671 & \\
\hline 201897_s_at & Hs. 374378 & CKS1B & 2.545476277 & Cell cycle - S/G2 \\
\hline 204170_s_at & Hs.83758 & CKS2 & 2.578149122 & Cell cycle - S/G2 \\
\hline 2028_s_at & Hs.654393 & $E 2 F 1$ & 1.344097502 & Cell cycle - G1/S \\
\hline 203692_s_at & Hs.269408 & E2F3 & 1.309684461 & Cell cycle - G1/S \\
\hline 200005_at & Hs.55682 & EIF3S7 & 0.584849774 & \\
\hline 204603_at & Hs.498248 & EX01 & 1.574862953 & \\
\hline 203358_s_at & Hs.444082 & $E Z H 2$ & 2.093540451 & Development \\
\hline 204767_s_at & Hs.409065 & FEN1 & 1.774057434 & DNA damage repair \\
\hline 218350_s_at & Hs.234896 & GMNN & 1.992657119 & \\
\hline 218662_s_at & Hs.567567 & HCAP-G & 1.97131609 & \\
\hline 204444_at & Hs. 8878 & KIF11 & 2.27997794 & \\
\hline 218755_at & Hs.73625 & KIF20A & 3.111335629 & \\
\hline 204709_s_at & Hs. 270845 & KIF23 & 1.459263584 & \\
\hline
\end{tabular}


Table S4: continued

\begin{tabular}{lllll}
\hline Probe ID & UniGene ID & Symbol & Fold Change $^{\mathbf{a}}$ & Biological Process $^{\mathbf{b}}$ \\
\hline 211519_s_at & Hs.69360 & KIF2C & 2.400629676 & \\
218355_at & Hs.438712 & KIF4A & 1.943634398 & Cell cycle - S/G2 \\
204162_at & Hs.414407 & KNTC2 & 2.289686738 & Cell cycle - S/G2 \\
203276_at & Hs.89497 & LMNB1 & 2.172895076 & \\
203362_s_at & Hs.591697 & MAD2L1 & 1.71344018 & Checkpoints \\
202107_s_at & Hs.477481 & MCM2 & 2.23767683 & DNA synthesis and replication \\
201555_at & Hs. 179565 & MCM3 & 1.714090705 & DNA synthesis and replication \\
201930_at & Hs.444118 & MCM6 & 2.460880373 & DNA synthesis and replication \\
212020_s_at & Hs.80976 & MKI67 & 2.203891304 & Cell cycle - S/G2 \\
209421_at & Hs.156519 & MSH2 & 1.750855393 & DNA damage repair \\
210947_s_at & Hs.280987 & MSH3 & 0.669034136 & \\
201710_at & Hs.179718 & MYBL2 & 1.637311714 & Cell cycle - G1/S \\
218039_at & Hs.615092 & NUSAP1 & 2.980337196 & \\
\hline
\end{tabular}

*Fold change (SCA/SBT)

\#Classification by Bracken et al. [17]

Table S5: Differentially expressed E2F targets (SAM) in SCA1s and SCA3s compared to SBTs

\begin{tabular}{ll}
\hline Expression (rel. to SBTs) & Differentially expressed E2F targets (Gene symbols) \\
\hline Higher expression in SCA1s & UBE2C, TK1, SLC16A1, RAD45L, PRC1, PLK1, MKI67, KNTC2, KIF2C, KIF20A, \\
& EXO1, E2F1, CFL1, CENPE, CENPA, CDK2, CCNA2 \\
Lower expression in SCA1s & TMEM59, TIMP1, RPS9, RPGR, PMM1, NBR1, MSH3, IPO7, EIF3S7, CCND3 \\
Higher expression in SCA3s & ZWINT, UBE2C, TTK, TOP2A, TK1, TIMELESS, TAF11, SMC4L1, SMC1L1, \\
& SMARCA3, SLC16A1, SKP2, SIL, RRM2, RFC4, RFC3, RAD54L, RAD51AP1, \\
& RAD51, PRC1, POLA2, PLK1, PCNA, PBK, NUSAP1, MYBL2, MSH2, MKI67, \\
& MCM6, MCM3, MCM2, MAD2L1, LMNB1, KNTC2, KIF4A, KIF2C, KIF23, KIF20A, \\
& KIF11, IMMT, HNRPAB, HCAP-G, GTPBP2, GMNN, FEN1, FBXO5, EZH2, EXO1, \\
& E2F3, CKS2, CKS1B, CFL1, CENPE, CENPA, CDC6, CDC25A, CDC20, CDC2, \\
& CCNA2, C210rf45, C12orf4, BUB1B, BUB1, BARD1, AURKB, ASK \\
Lower expression in SCA3s & RPS9, RPGR, EIF3S7
\end{tabular}

Table S6: TP53 exon 5-8 mutation analysis

\begin{tabular}{|c|c|c|c|c|c|c|c|}
\hline Case no & ID* & Diagnosis & Exon & Codon & Nucleotide change & Aminoacid change & Mutation type \\
\hline 30 & & SCA1 & 5 & 163 & TAC $>$ TGC & Tyr $>$ Cys & missense \\
\hline 45 & 49 & SCA3 & 5 & 175 & $\mathrm{CGC}>\mathrm{CAC}$ & Arg $>$ His & missense \\
\hline 57 & & SCA3 & 5 & 175 & $C G C>C A C$ & Arg $>$ His & missense \\
\hline 25 & 26 & SCA1 & 5 & 176 & $\mathrm{TGC}>\mathrm{TAC}$ & Cys $>$ Tyr & missense \\
\hline 34 & 39 & SCA3 & 6 & 195 & $A T C>A C C$ & Lie > Thr & missense \\
\hline 42 & 15 & SCA3 & 6 & 195 & ATC $>$ ACC & Lie $>$ Thr & missense \\
\hline 36 & 51 & SCA3 & 6 & 195 & ATC $>$ ACC & Lie $>$ Thr & missense \\
\hline 52 & & SCA3 & 6 & 204 & GAG > TAG & Glu $>$ stop & nonsense \\
\hline 55 & & SCA3 & 6 & 205 & TAT > TGT & Tyr $>$ Cys & missense \\
\hline 59 & & SCA3 & 6 & 214 & CAT > CGT & $\mathrm{His}>\mathrm{Arg}$ & missense \\
\hline 27 & 30 & SCA1 & 6 & 216 & GTG $>$ ATG & Val $>$ Met & missense \\
\hline 41 & 32 & SCA3 & 6 & 220 & TAT > TGT & Tyr $>$ Cys & missense \\
\hline 48 & & SCA3 & 6 & 220 & TAT > TGT & Tyr > Cys & missense \\
\hline 60 & & SCA3 & 7 & 241 & $-C$ & stop & deletion \\
\hline 40 & 11 & SCA3 & 7 & 249 & $A G G>G G G$ & Arg $>$ Gly & missense \\
\hline 51 & & SCA3 & 8 & 272 & GTG > ATG & Val > Met & missense \\
\hline
\end{tabular}

${ }^{*} \mathrm{D}$ as in Figure 2 in [13] and in the Gene Expression Omnibus 


\section{Supplementary figures}
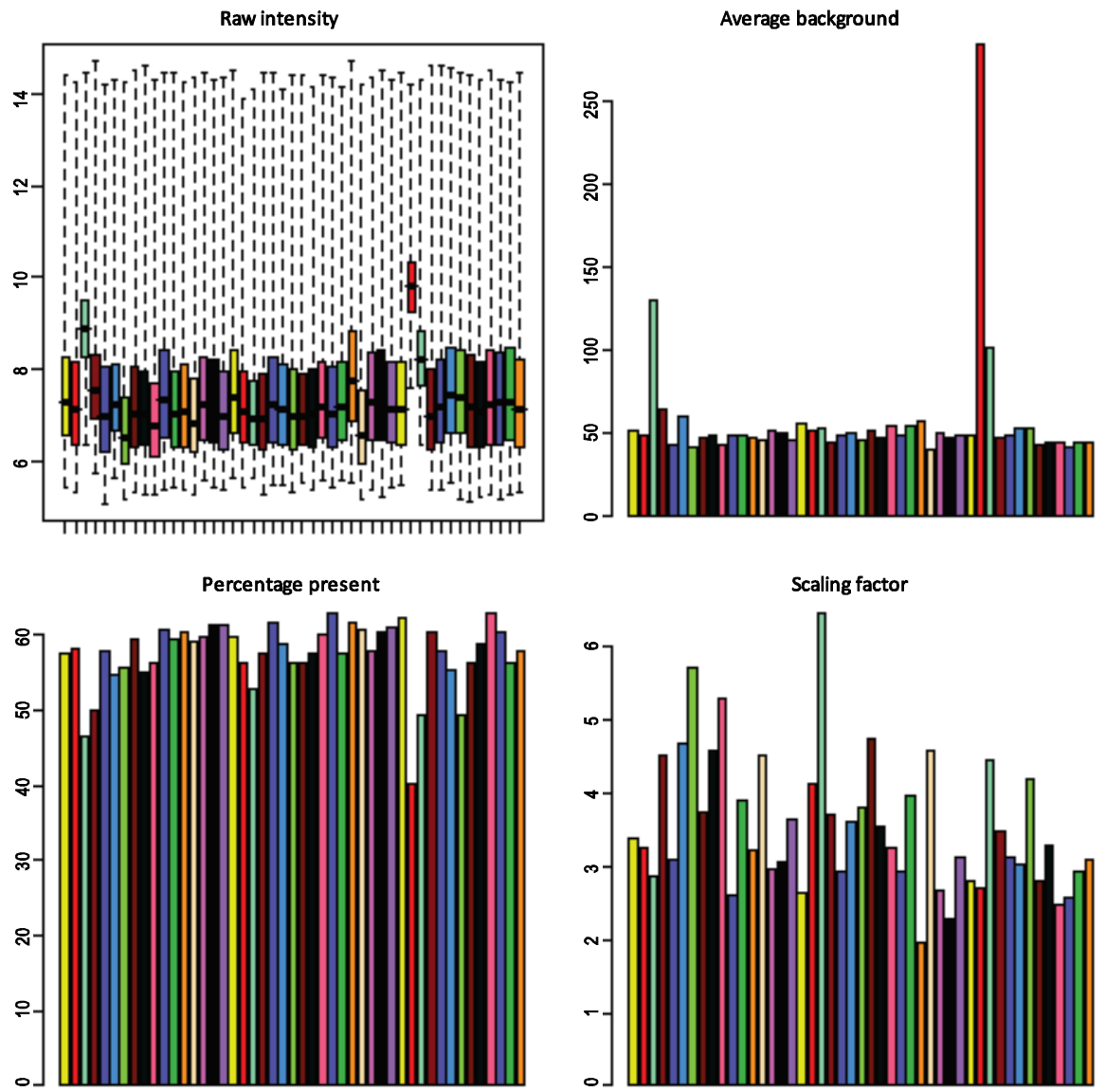

Hybridization controls
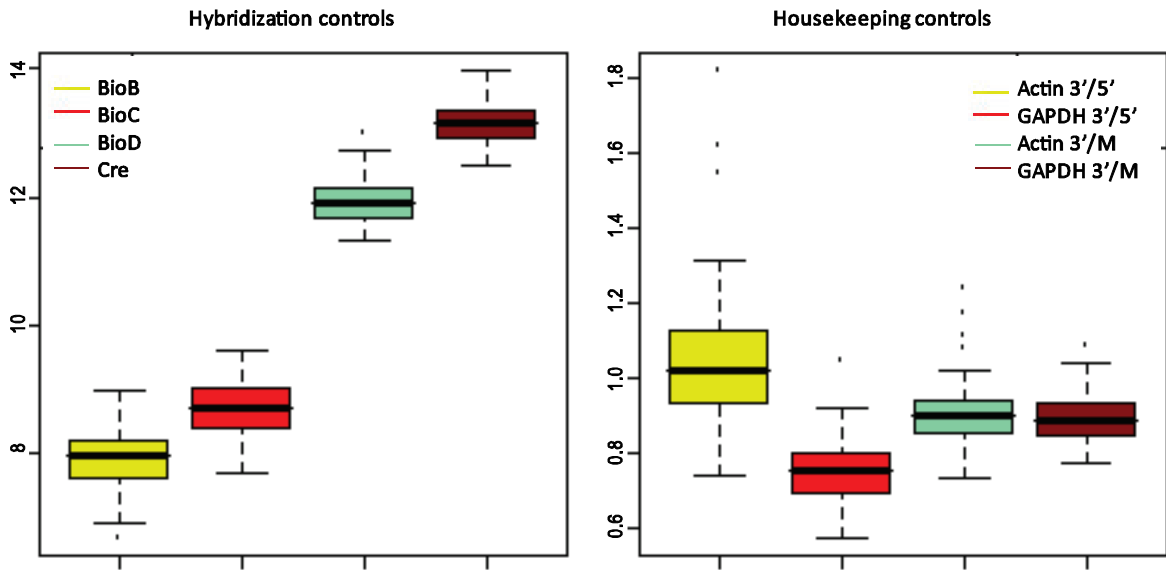

Figure S1: Microarray quality control

Raw intensities, average backgrounds, percentages of probes called present and scaling factors for different microarrays; results for hybridization controls and housekeeping controls. 
Delta vs. FDR

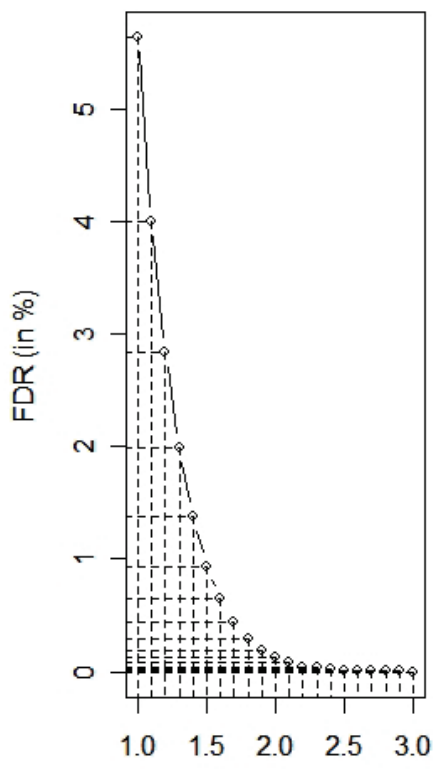

$\Delta$

\section{Delta vs. Significant Genes}

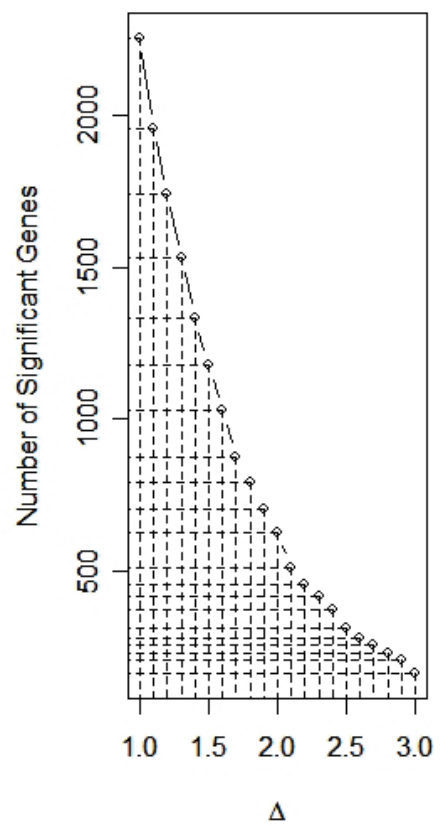

Figure S2A: Significance analysis of microarrays (SAM)

The false discovery rate (FDR, in \%) and the number of significant genes in function of the different values of delta $(\Delta)$.

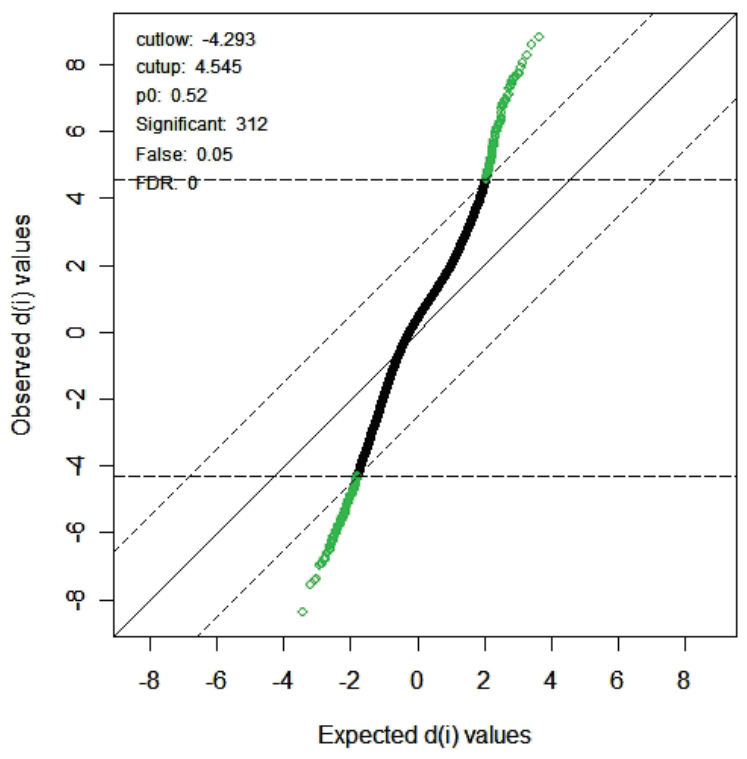

Figure S2B: SAM plot for delta $=\mathbf{2 . 5}$

Observed vs. expected relative difference in gene expression for the selected value of delta. 


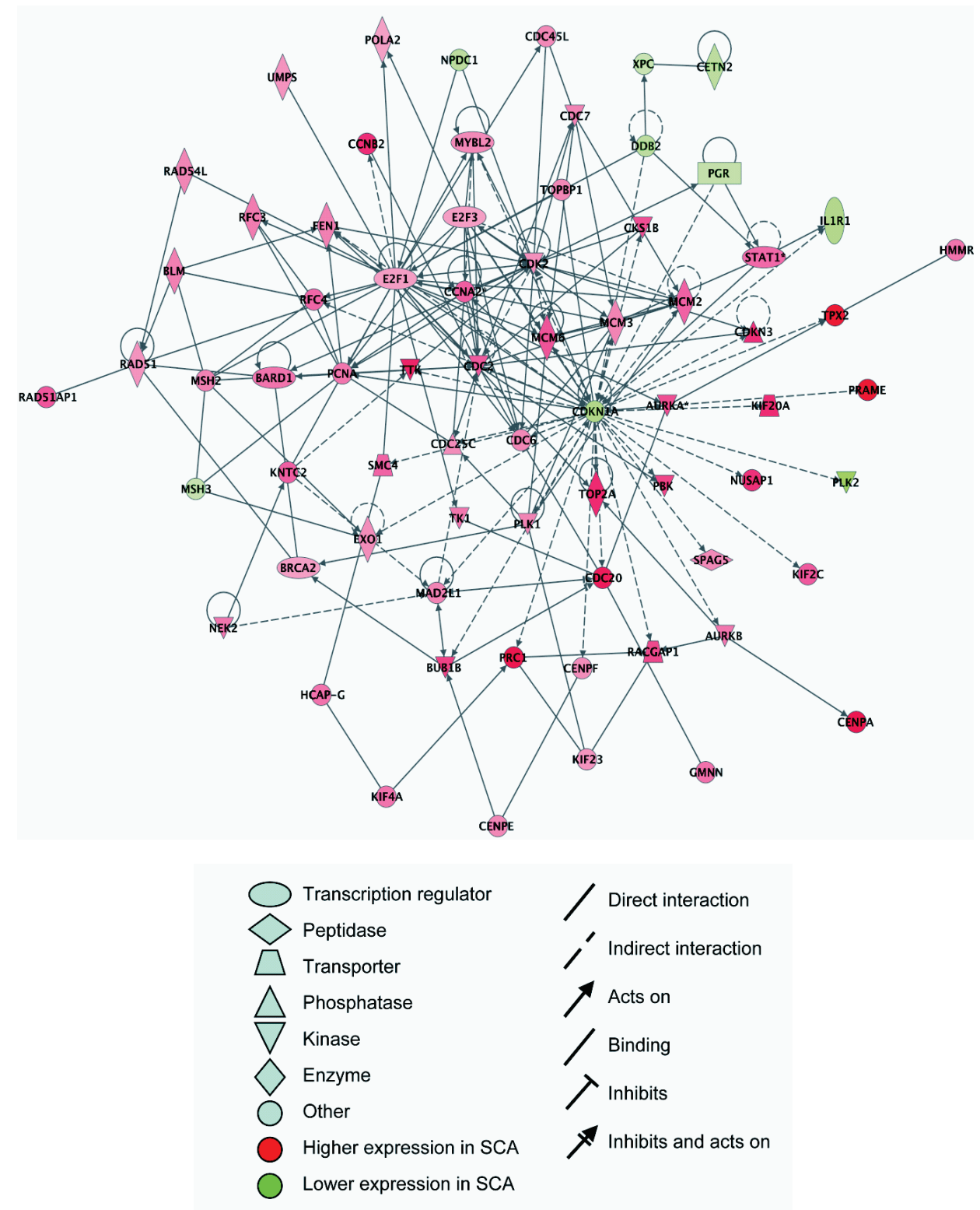

Figure S3: Ingenuity network

Ingenuity Pathway Analysis indicates central roles for E2F1 and CDKN1A, and supports E2F3 involvement. Lower and higher expression in SCAs is relative to SBTs. 


\section{Supplemental references}

13. Sieben NL, Oosting J, Flanagan AM, et al. Differential gene expression in ovarian tumors reveals Dusp 4 and Serpina 5 as key regulators for benign behavior of serous borderline tumors. J Clin Oncol 2005;23(29):7257-64.

17. Bracken AP, Ciro M, Cocito A, Helin K. E2F target genes: unraveling the biology. Trends in biochemical sciences 2004;29(8):409-17.

23. Tusher VG, Tibshirani R, Chu G. Significance analysis of microarrays applied to the ionizing radiation response. Proceedings of the National Academy of Sciences of the United States of America 2001;98(9):5116-21.

34. Silverberg SG. Histopathologic grading of ovarian carcinoma: a review and proposal. Int J Gynecol Pathol 2000;19(1):7-15. 


\section{CHAPTER 4}

\section{Decision tree analysis of copy number alterations identifies MECOM, LIMK1, NFKBIE, ACTN4, and NCOA3 as discriminators of serous ovarian borderline tumors and carcinomas}

Ingrid T.G.W. Bijsmans*, Marjolijn B. Verbruggen*, Marlies E. Nowee*, Jordy Coffa, Leander Van Neste, Kim M. Smits, Jan P. Schouten, Paul J. van Diest, Gerrit A. Meijer, D. Joop Kuik, Adriaan P. de Bruïne, Koen K. Van de Vijver, Nathalie L.G. Sieben, Rene H.M. Verheijen, Manon van Engeland, and Josephine C. Dorsman

* These authors contributed equally to this work

Submitted for publication 


\begin{abstract}
Serous ovarian borderline tumors (SBTs) and carcinomas (SCAs) differ substantially both in patient management and prognosis, necessitating correct diagnosis. Currently, the differential diagnosis is based primarily on histological criteria, while comprehensive tests based on molecular traits linked to carcinogenesis are lacking. Previously, we developed a Multiplex Ligation-Dependent Probe Amplification (MLPA) test interrogating ovarian cancer-related genes based on DNA profiling. Since SBTs and high-grade SCAs (HG-SCAs) are considered to be distinct entities, we hypothesized that analysis of their underlying molecular alterations by MLPA should facilitate discrimination. Here we developed two MLPA probe sets to analyze DNA copy number alterations of 32 genes (17 corresponding with pilot MLPA genes) on chromosomal arms $1,3 q, 6 p, 7 q, 8,12,16 p, 17 q, 19,20 q$ and $22 q$ in 41 SBTs and 45 HG-SCAs. SBTs and SCAs were found to cluster separately. In addition, decision tree analysis identified five key copy number alterations involving genes in metastasis and immune response pathways: gains of MECOM (MDS1 and EVI1 complex locus), LIMK1 (LIM domain Kinase 1), NFKBIE (NFKB Inhibitor Epsilon) and NCOA3 (Nuclear Receptor Coactivator 3) and loss of ACTN4 (Actinin alpha 4) correctly predicted SBT or SCA diagnosis in $96.5 \%$ (83/86) of cases. Cross-validation resulted in $88.4 \%$ accuracy. Of these markers, LIMK1 (invasion) and NFKBIE (immune response) have not been associated with ovarian cancer previously. This decision tree model may be used for molecular classification of SBTs and SCAs and for assisting pathologists in making the differential diagnosis. In addition, these data support the current idea that SBTs and HG-SCAs are unrelated entities.
\end{abstract}




\section{Introduction}

Epithelial ovarian cancer is a common and leading cause of death of gynecological malignancies in the Western world [1]. In 2008, 225,000 women were diagnosed with ovarian cancer and 140,000 women died of this disease worldwide [2]. Serous carcinomas (SCAs) are the most common epithelial ovarian tumors [3]. Due to nonspecific symptoms in early stages, the tumor has spread to the upper abdomen at time of diagnosis, resulting in advanced-stage SCA and poor prognosis [3, 4]. Long-term survival rates among patients with early-stage SCA range from $80-90 \%$, whereas the 5year survival rate of advanced-stage SCA is only $25 \%$. In contrast, serous borderline tumors (SBTs), an intermediate group of ovarian tumors characterized by varying nuclear atypia and lack of destructive stromal invasion, have a 5-year survival of 90$95 \%[3,5]$. SCAs are usually treated with radical surgery and adjuvant chemotherapy, while for SBTs a conservative surgical approach to maintain fertility in young women without chemotherapy is the treatment of choice. From a clinical and prognostic point of view a correct distinction between SBTs and SCAs is important. Previous studies showed that central pathology review can result in reclassification as borderline in approximately $30 \%$ of early-stage cases originally diagnosed as carcinomas [6]. Another study confirmed these results, changing the initial SCA diagnosis to SBT in $37.5 \%$ [7]. Misdiagnosis of SBTs as SCAs can lead to unnecessary and ineffective treatment of young SBT patients and increased health care costs [8]. Therefore, a sensitive and specific molecular diagnostic tool to assist the differential diagnosis can support pathologists. SBTs and high-grade SCAs (HG-SCA) can be considered as different molecular entities. Distinct molecular pathways have been proposed in the development of SBTs and SCAs $[9,10]$. Mutations in the oncogenes BRAF or KRAS are detected in $60 \%$ of SBTs [11, 12], whereas TP53 mutations are detected in up to $80 \%$ of HG-SCAs [13, 14]. Gene expression profiles clearly separate SBTs from SCAs [15-18]. Development of tools based on the molecular differences between SBTs and SCAs which can be readily implemented in diagnostic settings is warranted. We previously developed a MLPA probe set specifically tailored at grade $2 / 3$ serous fallopian tube carcinoma (FTC) and ovarian cancer-related genes based on array-CGH analysis [19]. We hypothesized that studying DNA alterations should facilitate discrimination of SBTs and SCAs and would aid diagnostics in the future. The aim of this study was to develop MLPA probe sets based on our previous DNA profiling studies to identify additional ovarian cancer DNA markers and to investigate whether the markers allow discrimination between SBTs and SCAs. 


\section{Materials and Methods}

\section{Patient material}

Formalin-fixed, paraffin-embedded or fresh-frozen tissue of 41 SBTs and 45 HG-SCAs (21 SCA2s and 24 SCA3s) was obtained from the VU University Medical Center (VUmc, Amsterdam); Maastricht University Medical Center (MUMC, Maastricht); Leiden University Medical Center (LUMC, Leiden); and Stichting PAMM (Eindhoven, The Netherlands). Haematoxylin and eosin slides were reviewed by expert gynecologic pathologists (PVD, KVdV, NS). None of the patients received chemotherapy prior to primary surgery. Clinicopathological characteristics are shown in Table 1. Baseline characteristics (International Federation of Gynaecology and Obstetrics (FIGO) stage, age at diagnosis and tumor grade) did not differ significantly between samples derived from the diverse hospitals, therefore the patient groups were considered as one (data not shown). Collection, storage and use of tissue and patient data were performed in agreement with the "Code for Proper Secondary Use of Human Tissue in The Netherlands" (http://www.fmwv.nl).

Table 1: Clinicopathological characteristics

\begin{tabular}{lll}
\hline Characteristics & & Patients (n=86) \\
\hline & SBT & $n(\%)$ \\
& SCA2 & $21(47.7)$ \\
& SCA3 & $24(27.4)$ \\
Age at diagnosis* & & \\
Years (range) & SBT & $42.9(19-65)$ \\
& SCA2 & $59.5(37-80)$ \\
& SCA3 & $62.1(40-79)$ \\
& & \\
FIGO stage* & & $n(\%)$ \\
SBT & stage I & $22(55.0)$ \\
& stage II & $6(15.0)$ \\
& stage III & $10(25.0)$ \\
SCA & stage IV & $2(5.0)$ \\
& & \\
& stage I & $5(11.1)$ \\
& stage II & $3(6.7)$ \\
& stage III & $31(68.9)$ \\
& stage IV & $6(13.3)$ \\
\hline
\end{tabular}

${ }^{*}$ Age and FIGO stage were unavailable of 1 SBT

\section{DNA isolation}

Tumor DNA of the VUmc population was extracted from 20 microdissected $10 \mu \mathrm{m}$ paraffin-embedded sections per specimen. DNA was prepared using the QIAmp ${ }^{\circledR}$ DNA mini kit (Qiagen $\mathrm{GmbH}$, Hilden, Germany) following a modification of the manufacturer's protocol [20]. DNA of the samples from LUMC, MUMC and Stichting PAMM was extracted using the Puregene DNA isolation kit (Gentra Systems, BIOzymTC; Landgraaf, The Netherlands) according to the manufacturer's description. DNA concentrations were determined spectrophotometrically (GeneQuant, Amersham Biosciences, Uppsala, Sweden; NanoDrop, Nanodrop technologies, Wilmington, USA). 
Multiplex Ligation-dependent Probe Amplification (MLPA)

MLPA was performed as described previously $[19,21]$. Probes were designed according to manufacturer's protocols (MRC Holland, Amsterdam, The Netherlands). Two probe sets were developed: P134B and P135B (Table 2), targeting regions on 1p, $1 q, 3 q, 6 p, 7 q, 8 p, 8 q, 12 p, 12 q, 16 p, 17 q, 19 p, 19 q, 20 q$, and 22q. Probe sequences are available on request. The probe sets were designed based on 1) previous array-CGH results; 2) pilot MLPA results in FTCs and SCAs [19]; and 3) literature-based genes of interest known to be involved in gynecological cancer. Probe set P134B contains 16 genes (28 probes) selected from literature and previous DNA profiling studies and 7 reference genes ( 8 probes; see Table 2 ) for quality control and normalization purposes selected from loci not showing alterations in our previous array-CGH study [19]. Probe set $\mathrm{P} 135 \mathrm{~B}$ includes 16 genes ( 31 probes) and 6 reference genes (9 probes). Of the 32 genes, 17 probes were present in the pilot MLPA set, whereas the remaining 15 are novel (Table 2). Samples were analyzed in triplicate if sufficient DNA was available. Reference runs performed on normal human female DNA from multiple donors (Promega, Madison, Wisconsin, USA) were included for normalization. MLPA reactions were performed using a Biometra thermocycler (Biometra, Gottingen, Germany), followed by product separation and quantification on a Beckman CEQ8000 capillary system (Beckman Coulter, Fullerton, USA). Data were imported into Coffalyser software [22] for data filtering and normalization. Data filtering assigns one specific signal from the data of each run to each probe known to be present in the MLPA mix. Sample signals were normalized to reference signals. The ratio of each MLPA probe between reference and sample was computed based on the combination of all control probes. Median probe ratios were used when samples were analyzed in triplicate, whereas the mean was used in samples performed in duplicate. For genes represented by two probes, the mean of the two probe results was used for calculations of gene aberrations. Ratios $<0.7$ were considered as loss, ratios $>1.3$ or $>2.0$ were considered gain or amplification, respectively.

\section{Data analysis}

Unsupervised hierarchical clustering was performed in $\mathrm{R}$, using the heatmap.2 package [23]. Losses are shown in red, gains in green and unaltered loci in black. A Manhattan distance measure was used to assess similarity/dissimilarity between samples. Genes and samples were clustered using Ward's algorithm. RapidMiner was used for decision tree generation and performance assessment using 'decision tree' and 'performance' algorithms. Due to limited sample size, MLPA data were not split into test and validation set. Cross-validation using the leave-one-out strategy was performed instead to achieve a more accurate measurement of the validity of the decision tree. The decision tree was constructed using the information gain ratio criterion and a minimal leaf size of 2 as main criteria. Associations between patient characteristics and the comparison of copy number alterations (CNAs) between SBTs and SCAs were calculated using Pearson Chi-Square test or Independent-Samples T Test where appropriate using SPSS 15.0. All $p$-values are two-sided, $p \leq 0.05$ was considered statistically significant. 
MLPA analysis revealed 5 key genes as discriminators between SBTs and SCAs

Table 2: MLPA probe sets

\begin{tabular}{|c|c|c|c|c|c|}
\hline Mix & Gene symbol & Locus* & $\begin{array}{l}\text { No of } \\
\text { probes }\end{array}$ & Array CGH\# & Literaturell \\
\hline \multirow[t]{23}{*}{ P134B } & PIK3CA & $3 q 26.32$ & 2 & & amp SCA $[31,58]$ \\
\hline & GL/3‡ & $7 p 14.1$ & 2 & no change & \\
\hline & CCM2‡ & $7 p 13$ & 1 & no change & \\
\hline & LIMK1 & $7 q 11.23$ & 2 & gain SCA & \\
\hline & FGFR1 & $\begin{array}{l}\text { 8p11.23- } \\
\text { p11.22 }\end{array}$ & 2 & & gain SCA [28] \\
\hline & $M Y C$ & $8 q 24.21$ & 2 & gain SCA; amp FTC, SCA & \\
\hline & PTK2 & $8 q 24.3$ & 2 & gain SCA; amp FTC, SCA & \\
\hline & EXT2‡ & $11 p 11.2$ & 1 & no change & \\
\hline & BEST1 $\ddagger$ & $11 q 12.3$ & 1 & no change & \\
\hline & KDM5A & $12 p 13.33$ & 2 & amp FTC, SCA & \\
\hline & TSPAN9 & $\begin{array}{l}\text { 12p13.33- } \\
\text { p13.32 }\end{array}$ & 1 & gain SCA; amp FTC, SCA & \\
\hline & MDM2 & $12 q 15$ & 2 & & $\begin{array}{l}\text { gain and over-exp } \\
\text { SCA }[28,59]\end{array}$ \\
\hline & SOCS1 & $16 p 13.13$ & 1 & & [27] \\
\hline & OMG & $17 q 11.2$ & 1 & no change & \\
\hline & ERBB2 & $17 q 12$ & 2 & amp FTC, SCA & \\
\hline & SMARCA4 & $19 p 13.2$ & 2 & gain FTC, SCA; amp FTC & \\
\hline & $A K T 2$ & $19 q 13.2$ & 2 & & amp SCA [31] \\
\hline & ZNF337‡ & 20p11. 1 & 1 & no change & \\
\hline & PYGB $\ddagger$ & 20p11.21 & 1 & no change & \\
\hline & $B C L 2 L 1$ & $20 q 11.21$ & 2 & gain SCA; amp SCA & \\
\hline & NCOA3 & $20 q 13.12$ & 1 & gain SCA; amp FTC & \\
\hline & PTPN1 & $20 q 13.13$ & 1 & gain SCA; amp FTC, SCA & \\
\hline & KCNQ2 & $20 q 13.33$ & 2 & gain SCA; amp FTC, SCA & \\
\hline \multirow[t]{22}{*}{ P135B } & MYCBP & $1 p 34.3$ & 2 & & [25] \\
\hline & PSMB4 & $1 q 21.3$ & 2 & gain SCA; amp SCA & \\
\hline & COL3A1 & $2 q 32.2$ & 1 & no change & \\
\hline & MECOM & $3 q 26.2$ & 2 & amp FTC, SCA & \\
\hline & NFKBIE & $6 p 21.1$ & 2 & amp SCA & \\
\hline & GL/3‡ & $7 p 14.1$ & 3 & no change & \\
\hline & MTSS1 & $8 q 24.13$ & 2 & gain SCA; amp FTC, SCA & \\
\hline & RNF139 & $8 q 24.13$ & 2 & amp FTC, SCA & \\
\hline & EIF2C2 & $8 q 24.3$ & 2 & gain SCA; amp FTC, SCA & \\
\hline & $A L X 4 \ddagger$ & $11 p 11.2$ & 2 & no change & \\
\hline & EXT2‡ & $11 \mathrm{p} 11.2$ & 1 & no change & \\
\hline & BEST1 & $11 q 12.3$ & 1 & no change & \\
\hline & TEAD4 & $12 p 13.33$ & 2 & SCA; amp FTC, SCA & \\
\hline & GPRC5A & $12 p 13.1$ & 2 & gain SCA; amp FTC, SCA & \\
\hline & OMG & $17 q 11.2$ & 1 & no change & \\
\hline & CCNE1 & $19 q 12$ & 1 & amp FTC, SCA & \\
\hline & DPF1 & $\begin{array}{l}19 q 13.13- \\
q 13.2\end{array}$ & 2 & gain FTC; amp FTC & \\
\hline & ACTN4 & $19 q 13.2$ & 2 & gain FTC; amp FTC & \\
\hline & SPINT2 & $19 q 13.2$ & 2 & gain FTC; amp FTC & \\
\hline & BCAS4 & $20 q 13.13$ & 2 & amp FTC, SCA & \\
\hline & NFATC2 & $20 q 13.2$ & 2 & & $\begin{array}{l}\text { over-exp breast } \\
\text { cancer [26] }\end{array}$ \\
\hline & PDGFB & $22 q 13.1$ & 2 & & amp in SCA [24] \\
\hline
\end{tabular}

${ }^{*}$ The chromosome positions were determined with the February 2009 Assembly of the UCSC Genome Browser \#Genes chosen based on our previous array-CGH analysis and pilot MLPA study [19]

|| Literature-based genes

†Reference probes: these probes were unaltered in the analyzed FTCs and SCAs [19]

Abbreviations: amp: high-level amplification; over-exp: over-expression; gain: recurrent gain; FTC: fallopian tube carcinoma; SCA: serous ovarian carcinoma 


\section{Results}

Tailored MLPA sets for SCA2s/SCA3s

To identify DNA markers for serous gynecological malignancies, we developed two MLPA probe sets based on our previous array-CGH analyses showing either recurrent gain and/or high-level amplification [19], extended with genes of interest from literature [24-28]. The P134B and P135B probe sets contain 32 genes: 15 new genes were added to the 17 genes present in our pilot MLPA set [19]. Only genes of interest that had already been linked to molecular oncogenesis were included. Control probes targeting gene regions which were found unaltered in our previous array-CGH studies were also included, facilitating accurate calculations of gene changes.

\section{SCAs show significantly more CNAs than SBTs}

Thirty-two genes were analyzed for CNAs in 41 SBTs and 45 SCAs. The total number of gains and losses was significantly lower in SBTs as compared to SCAs for most (25/32) of the analyzed genes (Table 3). The mean number of gains was 4.1 in SBTs and 9.0 in SCAs $(p<0.001)$. The mean number of losses was significantly lower in SBTs (1.0) as compared to SCAs (1.8) $(\mathrm{p}=0.008)$. Differences were not identified when analyzing CNAs between SCA2s and SCA3s, except for MYC, which showed significantly more gains in SCA3s $(20 / 24 ; 83.3 \%)$ as compared to SCA2s (11/21 (52.4\%); $p=0.025)$. Loss of MDM2 (4/41 (9.8\%) of SBTs and 20/45 (44.4\%) of SCAs), ERBB2 (1/41 (2.4\%) and 13/45 (28.9\%) respectively) and $A K T 2(3 / 41(7.3 \%)$ and $3 / 45(6.7 \%))$ were identified, although overexpression and/or amplification of these genes have been detected previously [29-31]. The pro-survival anti-apoptotic protein BCL2L1 was gained in 23/45 (51.1\%) SCAs and lost in the remaining 22 cases (48.9\%). Genes known to be involved in serous ovarian tumorigenesis, and identified to be significantly gained in SCAs as compared to SBTs included MECOM (SCA 19/45 (42.2\%) versus 0\% in SBTs), PIK3CA (15/45 (33.3\%) versus 2/41 (4.9\%)), MYC (31/45 (68.9\%) versus 12/41 (29.3\%)), CCNE1 (14/45 (31.1\%) versus $2 / 41(4.9 \%))$, ACTN4 (8/45 (17.8\%) versus $0 \%)$, and NCOA3 (6/45 (13.3\%) versus $0 \%)$. Table 3 shows that amplifications were more frequently detected in SCAs as compared to SBTs. MYCBP, MTSS1, MYC, and RNF139 were the only genes amplified in SBTs. SCAs harbored amplifications of all genes, except LIMK1, MDM2 and NCOA3. In conclusion, fewer CNAs were detected in SBTs as compared to SCAs for the analyzed genes. 
Table 3: CNAs in SBTs and SCAs as measured by MLPA

\begin{tabular}{|c|c|c|c|c|c|c|c|}
\hline \multirow[t]{2}{*}{ Gene } & \multicolumn{3}{|c|}{ SBT $(n=41)$} & \multicolumn{3}{|c|}{$\operatorname{SCA}(n=45)$} & \multirow[t]{2}{*}{$p$-value* } \\
\hline & gain (\%) & amp & loss (\%) & gain (\%) & $\mathrm{amp}$ & loss (\%) & \\
\hline MYCBP & $2(4.9)$ & 1 & $1(2.4)$ & $15(33.3)$ & 1 & $3(6.7)$ & 0.002 \\
\hline PSMB4 & 0 & 0 & $1(2.4)$ & $17(37.8)$ & 4 & 0 & $<0.001$ \\
\hline MECOM & 0 & 0 & $2(4.9)$ & 19 (42.2) & 4 & $2(4.4)$ & $<0.001$ \\
\hline PIK3CA & $2(4.9)$ & 0 & $1(2.4)$ & 15 (33.3) & 3 & $2(4.4)$ & 0.003 \\
\hline NFKBIE & 0 & 0 & 0 & $25(55.6)$ & 3 & $1(2.2)$ & $<0.001$ \\
\hline LIMK1 & $2(4.9)$ & 0 & 0 & $7(15.6)$ & 0 & $1(2.2)$ & NS \\
\hline FGFR1 & 7 (17.1) & 0 & 0 & $14(31.1)$ & 3 & $3(6.7)$ & NS \\
\hline RNF139 & $14(34.1)$ & 2 & 0 & 32 (71.1) & 11 & 0 & 0.001 \\
\hline MTSS1 & $6(14.6)$ & 1 & 0 & $24(53.3)$ & 4 & 0 & $<0.001$ \\
\hline MYC & $12(29.3)$ & 1 & 0 & $31(68.9)$ & 13 & 0 & $<0.001$ \\
\hline EIF2C2 & $7(17.1)$ & 0 & 0 & $24(53.3)$ & 5 & $2(4.4)$ & $<0.001$ \\
\hline PTK2 & $12(29.3)$ & 0 & $1(2.4)$ & $16(35.6)$ & 4 & 0 & NS \\
\hline JARID1A & $3(7.3)$ & 0 & $1(2.4)$ & $18(40.0)$ & 2 & $1(2.2)$ & 0.002 \\
\hline TEAD4 & $9(22.0)$ & 0 & 0 & $21(46.7)$ & 6 & $1(2.2)$ & 0.029 \\
\hline TSPAN9 & 7 (17.1) & 0 & 0 & $18(40.0)$ & 4 & $2(4.4)$ & 0.018 \\
\hline GPRC5A & $3(7.3)$ & 0 & 0 & $16(35.6)$ & 1 & $2(4.4)$ & 0.002 \\
\hline MDM2 & 0 & 0 & $4(9.8)$ & 0 & 0 & $20(44.4)$ & $<0.001$ \\
\hline SOCS1 & $1(2.4)$ & 0 & 0 & $10(22.2)$ & 1 & $5(11.1)^{\prime}$ & 0.001 \\
\hline ERBB2 & $2(4.9)$ & 0 & $1(2.4)$ & $4(8.9)$ & 2 & $13(28.9)$ & 0.002 \\
\hline SMARCA4 & $2(4.9)$ & 0 & $1(2.4)$ & $3(6.7)$ & 0 & $7(15.6)$ & NS \\
\hline CCNE1 & $2(4.9)$ & 0 & $2(4.9)$ & $14(31.1)$ & 5 & $1(2.2)$ & 0.007 \\
\hline DPF1 & $6(14.6)$ & 0 & 0 & $19(42.2)$ & 3 & $1(2.2)$ & 0.010 \\
\hline SPINT2 & $1(2.4)$ & 0 & $1(2.4)$ & $7(15.6)$ & 2 & $2(4.4)$ & NS \\
\hline ACTN4 & 0 & 0 & 0 & $8(17.8)$ & 1 & 5 (11.1) & 0.001 \\
\hline AKT2 & 0 & 0 & $3(7.3)$ & $5(11.1)$ & 1 & $3(6.7)$ & NS \\
\hline$B C L 2 L 1$ & $4(9.8)$ & 0 & 0 & $23(51.1)$ & 3 & $22(48.9)$ & $<0.001$ \\
\hline NCOA3 & 0 & 0 & $3(7.3)$ & $6(13.3)$ & 0 & $1(2.2)$ & 0.033 \\
\hline PTPN1 & 0 & 0 & $5(12.2)$ & 7 (15.6) & 1 & $1(2.2)$ & 0.009 \\
\hline BCAS4 & $1(2.4)$ & 0 & $1(2.4)^{\prime}$ & $13(28.9)$ & 2 & 0 & 0.003 \\
\hline NFATC2 & $2(4.9)$ & 0 & $1(2.4)$ & $13(28.9)$ & 2 & 0 & 0.009 \\
\hline KCNQ2 & $5(12.2)$ & 0 & $1(2.4)$ & $15(33.3)$ & 4 & $1(2.2)$ & NS \\
\hline PDGFB & 0 & 0 & $1(2.4)$ & $1(2.2)$ & 1 & $10(22.2)$ & 0.013 \\
\hline
\end{tabular}

${ }^{*} \mathrm{p}$-value (T-test) gain and loss SBT versus SCA; NS: not significant

\section{Majority of SBTs cluster separately from SCAs}

Unsupervised hierarchical clustering of CNAs of 32 genes showed that the majority of the 41 SBTs and 45 SCAs cluster separately, except for 2 SBTs which cluster with SCAs and 12 SCAs (8 SCA2s and 4 SCA3s) which cluster with SBTs (Figure 1). The 2 SBTs that clustered with the SCAs showed more gains as compared to the mean gains in SBTs: 6 and 10 gains versus 4.1 in SBT. 10/12 SCAs clustering with the SBTs showed fewer gains (mean 4.3) and altered number of losses (mean 2.3) as compared to the mean gain of 9.0 and mean loss of 1.8 in SCAs. Review of the H\&E slides of separately clustering tumors confirmed the initial diagnoses as SCAs. 


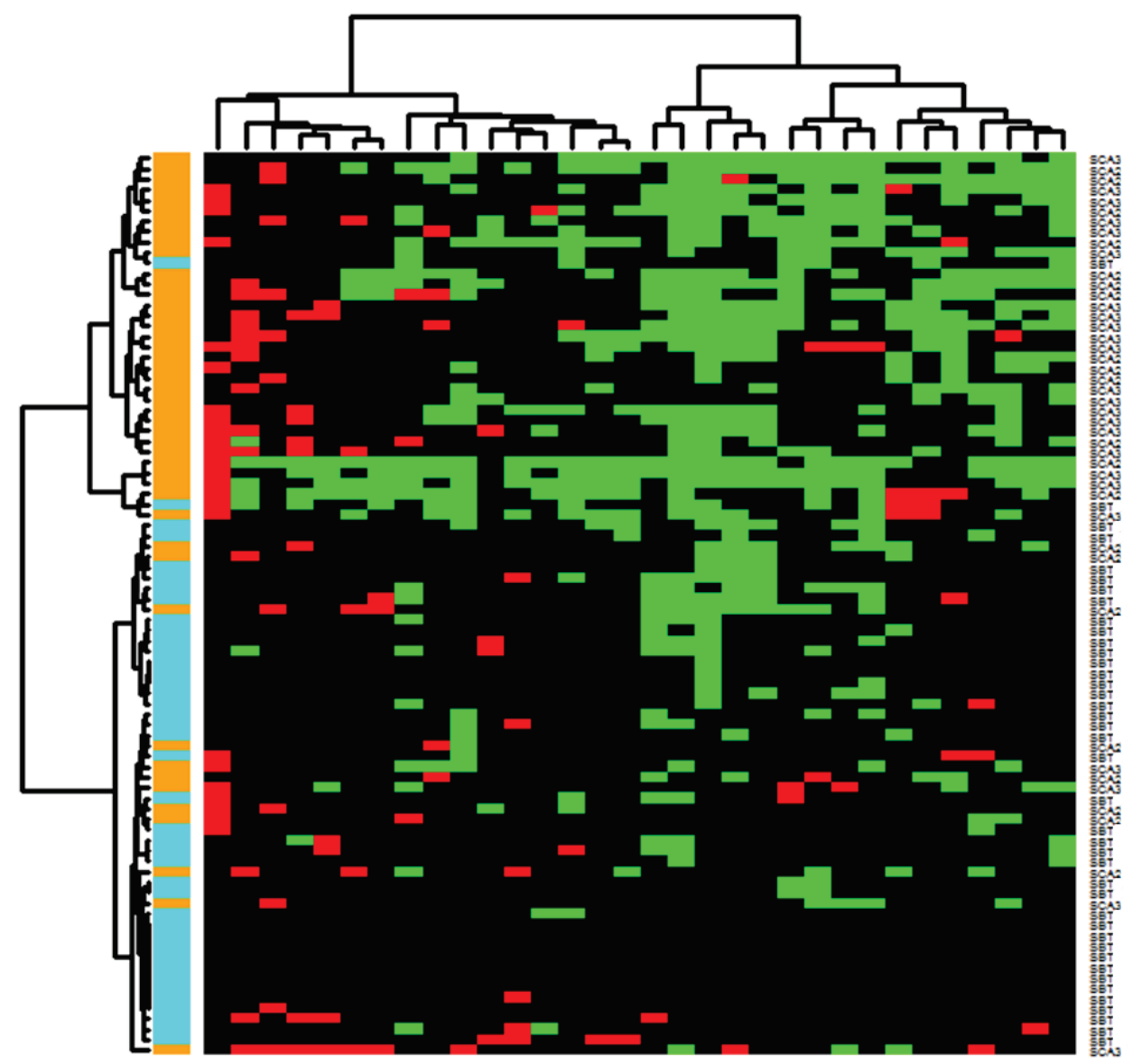

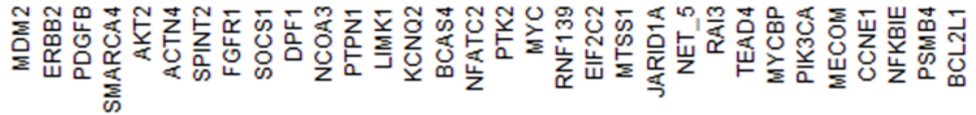

Figure 1: Unsupervised hierarchical cluster analysis of 86 serous ovarian tumors

Hierarchical clustering of 32 genes in 41 SBTs and 45 SCAs is shown. Each row represents a tumor, single genes are displayed in columns. Losses are shown in red, gains in green and unaltered loci in black. 
Decision tree analysis identifies MECOM, LIMK1, NFKBIE, ACTN4, and NCOA3 as discriminators of SBTS and SCAs

To investigate whether a more accurate prediction of SBTs and SCAs could be achieved based on MLPA data, we performed decision tree analysis (Figure 2). A combination of CNAs of 5 genes, i.e. MECOM, LIMK1, NFKBIE, ACTN4 and NCOA3 was able to correctly predict a tumor sample to be a SBT or a SCA in $96.5 \%$ of cases.

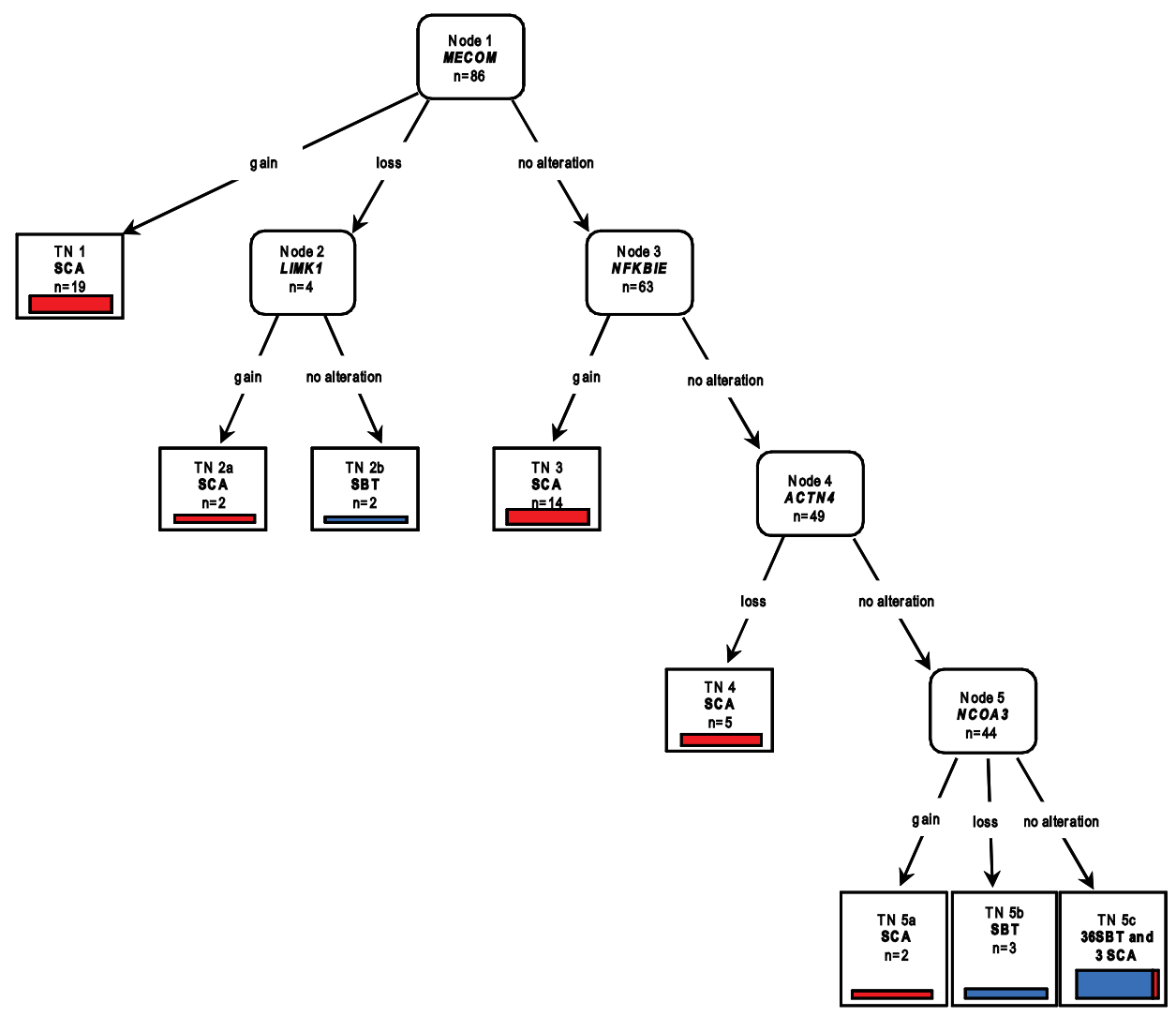

Figure 2: Decision tree: SBTs can be distinguished from SCAs based on CNAs of MECOM, LIMK1, NFKBIE,

\section{ACTN4, and NCOA3}

The decision tree shows that alterations in the five key genes MECOM, LIMK1, NFKBIE, ACTN4, and NCOA3 accurately predict whether a tumor is a SBT or a SCA. MECOM is displayed as the root node (node 1). Splitting at MECOM results in a terminal node (depicted with TN; square) in case of a gain, node 2 (LIMK1) in case of loss, or node 3 (NFKBIE) if not altered. Unaltered NFKBIE splits in node 4 (ACTN4), subsequently split in node 5 (NCOA3) and terminal nodes. In each terminal node the number of samples within this node is depicted. Red bars represent SCAs, bleu bars SBTs.

Branch points were chosen relative to the largest gain in samples for a specific class. All samples $(n=86)$ were included in this model. MECOM is displayed as the root node (Node 1). Splitting was based on gain of MECOM, indicating that a sample with a gain was send to the terminal node 1 (TN1) and was classified as a SCA. Loss of MECOM was detected in 2 SCAs and 2 SBTs. These cases were placed in node 2, displaying LIMK1 which was gained in the SCAs and unaltered in the SBTs (TN2a and TN2b). Samples 
without alteration of MECOM were sent to node 3: NFKBIE. Gain of NFKBIE generated the terminal node TN3 containing 14 SCAs, whereas unaltered NFKBIE was subsequently split in node 4 (ACTN4). Loss of ACTN4 was associated with SCAs $(n=5)$ resulting in terminal node TN4. ACTN4 without genetic alterations was split in node 5 (NCOA3), branching in the terminal nodes TN5a (gain; 2 SCAs), TN5b (loss; 3 SBTs) or TN5c (no alteration; 36 SBTs and 3 SCAs). Node TN5c contains three misclassified SCAs. Altogether, decision tree analysis resulted in a global accuracy of $96.5 \%(83 / 86$ samples were correctly classified). Cross-validation resulted in $88.4 \%$ accuracy, with class precisions (defined as true positives/(true positives plus false positives); positive predictive value) of $83.0 \%$ and $94.9 \%$ and class recall (true positives/(true positives plus false negatives); sensitivity) of $95.1 \%$ and $82.2 \%$ for SBTs and SCAs respectively. We and others have previously detected CNAs of MECOM, ACTN4 and NCOA3 in ovarian cancer [19, 32-36], whereas LIMK1 and NFKBIE are novel candidate serous ovarian cancer genes (Table 4).

Table 4: Genetic alterations of key genes MECOM, LIMK1, NFKBIE, ACTN4, and NCOA3 in our study and previously published results

\begin{tabular}{|c|c|c|c|c|c|c|}
\hline \multirow[t]{2}{*}{ Gene } & \multicolumn{2}{|c|}{ Gain (\%) } & \multicolumn{2}{|c|}{ Loss (\%) } & \multirow[t]{2}{*}{ Literature (method) } & \multirow[t]{2}{*}{ References } \\
\hline & SBT & SCA & SBT & SCA & & \\
\hline \multirow[t]{2}{*}{ MECOM } & 0 & $19 / 45$ & $2 / 41$ & $2 / 45$ & MECOM gain detected in $43 \%$ of SCAs & [34] \\
\hline & & $(42.2)$ & $(4.9)$ & $(4.4)$ & $\begin{array}{l}\text { MECOM gain significantly more } \\
\text { detected in chemo resistant SCAs }\end{array}$ & [33] \\
\hline \multirow[t]{2}{*}{ LIMK1 } & $2 / 41$ & $7 / 45$ & 0 & $1 / 45$ & Associations between LIMK1 and & \\
\hline & $(4.9)$ & $(15.6)$ & & $(2.2)$ & $\begin{array}{l}\text { serous ovarian cancer have not been } \\
\text { published }\end{array}$ & \\
\hline NFKBIE & 0 & $\begin{array}{l}25 / 45 \\
(55.6)\end{array}$ & 0 & $\begin{array}{l}1 / 45 \\
(2.2)\end{array}$ & $\begin{array}{l}\text { Associations between NFKBIE and } \\
\text { serous ovarian cancer have not been } \\
\text { published }\end{array}$ & \\
\hline ACTN4 & 0 & $\begin{array}{l}8 / 45 \\
(17.8)\end{array}$ & 0 & $\begin{array}{l}5 / 45 \\
(11.1)\end{array}$ & $\begin{array}{l}\text { Increased copy number in } 17.6 \% \text { of } \\
\text { advanced-stage SCAs (FISH) }\end{array}$ & [36] \\
\hline \multirow[t]{2}{*}{ NCOA3 } & 0 & $6(13.3)$ & $\begin{array}{l}3 / 41 \\
(7.3)\end{array}$ & $\begin{array}{l}1 / 45 \\
(2.2)\end{array}$ & $\begin{array}{l}\text { Amplification in } 9 / 122(7.4 \%) \text { of } \\
\text { sporadic ovarian tumours; subtype not } \\
\text { specified (Southern blot) }\end{array}$ & [32] \\
\hline & & & & & $\begin{array}{l}\text { Amplification in } 25 \% \text { of sporadic SCAs } \\
\text { (FISH) }\end{array}$ & [35] \\
\hline
\end{tabular}

\section{Discussion}

In this study, two MLPA probe sets based on our previous array-CGH analysis [19] and specifically tailored at HG-SCAs, were developed. Besides known genes in ovarian carcinogenesis (AKT2, FGFR1, PDGFB, and PIK3CA), new putative relevant markers (BCL2L1, DPF1, KCNQ2, LIMK1, MYCBP, NFATC2, PTPN1, SMARCA4, and SOCS1) were identified. We compared the MLPA data of HG-SCAs with those of SBTs and present evidence that based on copy number analysis of 32 genes, SBTs and SCAs clustered separately, except for 2/41 (4.9\%) SBTs and 12/45 (26.7\%) SCAs, lending further support to the model that these tumors are distinct entities [37]. Furthermore, decision tree analysis identified CNAs in five genes that accurately discriminated between SBTs and SCAs in $96.5 \%$ of cases. The 3 SCAs that misclassified with decision tree analysis also clustered with the SBTs and were all SCA2s, FIGO stage IIIC. For each of the 3 SCAs, only one loss was detected, whereas the number of gains was 1, 3 and 7 
respectively, which is less as compared to the mean number of losses (1.8) and gains (9.0) in SCAs. It is therefore not unexpected that, based on copy number profiles, these samples were classified as SBTs. Decision tree analysis, as was performed on CNAs in this study, is currently being introduced into clinical pathology practice to facilitate morphologic and immunohistochemical diagnosis of lymphoma subtypes [38] and to guide treatment decisions based on the molecular classification of lung cancer patients (mutation and amplification analysis of KRAS, EGFR and MET) [39]. This type of analysis takes into account the underlying molecular biology of carcinogenesis and might therefore aid diagnostic and treatment decisions. In this study we identified key copy number alterations of MECOM, LIMK1, NFKBIE, ACTN4, and NCOA3, genes known to be involved in immune response, migration and invasion, the latter being the main discriminator between SBTs and SCAs. MECOM (3q26.2) encodes a nuclear zinc finger DNA-binding protein [40] involved in inhibition of TGF $\beta$ signaling and amplified in approximately half of SCAs [33, 34, 41, 42]. Evidence for oncogenic functioning comes from in vitro experiments showing that MECOM overexpression increased cell proliferation and migration [41]. Since MECOM was gained in SCAs only, this gene might be a functionally relevant candidate gene in serous ovarian cancer and used as a single marker in the discrimination between SBTs and SCAs. LIMK1 (7q11.23) is a dual specificity kinase family member involved in cytoskeleton reorganization, an essential aspect of motility and invasion of cancer cells [43]. LIMK1 overexpression upregulates urokinase-type plasminogen activator (UPA), a serine protease facilitating extracellular matrix degradation and tumor cell invasion [44]. Increased UPA expression due to LIMK1 activity results in tumor growth in mice, tumor angiogenesis and metastasis [45]. Involvement of this pathway in serous ovarian cancer has been reported previously $[18,46]$. The invasive phenotype of SCAs might be explained by LIMK1 gain followed by UPA activity and subsequent destructive invasion, a phenomenon not present in SBTs. LIMK1 is an interesting gene in serous ovarian cancer since gains are primarily detected in SCAs $(7 / 45 ; 15.6 \%)$ as compared to SBTs $(2 / 41 ; 4.9 \%)$. This gene is, nevertheless, not useful as a single marker, but might perform well in combination with other genes as in the proposed model. NFKBIE (6p21.1) is an inhibitor of NFKB, a family of transcription factors involved in cell survival, inflammation and immune response. Activation of $N F K B$ signaling due to degradation of its inhibitor results in nuclear accumulation and transcription of target genes [47, 48]. Upregulation of NFKBIE and subsequent $N F K B$ repression may result in downregulation of MHC class I genes and immune response escape, indicating that NFKBIE might also constitute a relevant marker. ACTN4 (19q13.2) is an isoform of non-muscular $\alpha$-actinin enhancing motility and invasion by actin cytoskeleton remodeling in ovarian cancer cells [36, 49, 50]. ACTN4 binds directly to PI3Ks regulatory subunit $p 85$ and $A K T 1$, genes involved in cell proliferation [51,52]. Expression of ACTN4 mRNA and protein was associated with advanced and metastatic ovarian cancer [50,53]. Gain of ACTN4 was also detected in SCAs indicating that ACTN4 gain might be useful as a discriminative marker, although in the current decision tree model ACTN4 loss is exploited for prediction. NCOA3 (20q13.12) is a member of the steroid receptor coactivator family involved in transcriptional activation of nuclear hormone receptors and transcription factors [54], amongst which E2F1. A positive feedback-loop between NCOA3 and E2F1 to sustain high gene activity and promote proliferation and tumorigenesis has been reported for 
breast cancer [55]. Previously, we reported higher E2F1 mRNA expression levels in SCAs in comparison to SBTs [56]. NCOA3 activation in SCAs might be caused by copy number gain, as shown in the present study and/or due to E2F1 activation and subsequent NCOA3 overexpression. Qin et al. showed that NCOA3 expression in human breast cancer cells induces expression of the matrix metalloproteinases MMP2 and MMP9, resulting in tumor cell invasion and metastasis due to degradation of the extracellular matrix [57]. Identification of MECOM, LIMK1, NFKBIE, ACTN4, and NCOA3 by decision tree analysis indicates that copy number analysis of these five genes by MLPA is a potential molecular and diagnostic tool to aid the diagnosis and to minimize misdiagnoses which can amount to approximately $30 \%$ of early-stage ovarian tumors $[6,7]$. In this study, morphologically well-defined SBTs and HG-SCAs were analyzed for CNAs. Although we performed cross-validation to evaluate the accuracy of decision tree analysis, validation in larger retrospective and prospective independent series, including low-grade SCAs (LG-SCAs), is required. A molecular tool to correctly classify SBTs and SCAs will result in 1) a decrease of misdiagnoses; 2) a decline in overtreatment of patients and the consequences thereof; and 3) a subsequent reduction in hospitalization costs. Eventually, the clinical value of this test will be determined by the quality of life of the young women suffering from SBTs which will be treated accordingly.

In summary, we developed two MLPA probe sets specifically tailored at HG-SCAs, and new putative relevant marker genes for serous ovarian cancer were identified. Furthermore, we provide additional evidence that SBTs and HG-SCAs are unrelated entities. Besides a better understanding of the pathogenesis of SBTs and HG-SCAs, MLPA probe sets based on genomic analyses may improve accurate diagnosis of individual tumors in the future.

\section{Acknowledgements}

We thank A.M. Croonen, H. Hollema, R. Montironi, J.R. Salisbury, H. Stel, A.G.J. van der Zee, and T. Boerman for providing specimens. We also thank R. Brekelmans and N. Laddach for assistance with the MLPA analysis. M.B. Verbruggen and M.E. Nowee were supported by a grant of the Biocare foundation. 


\section{References}

1. Cannistra SA. Cancer of the ovary. N Engl J Med 2004;351(24):2519-29.

2. Ferlay J, Shin HR, Bray F, Forman D, Mathers C, Parkin DM. Estimates of worldwide burden of cancer in 2008: GLOBOCAN 2008. Int J Cancer 2010;127(12):2918-27.

3. Prat J. Surface epithelial-stromal tumors: Serous tumors. Saunders; 2004: 83-111

4. Holschneider $\mathrm{CH}$, Berek JS. Ovarian cancer: epidemiology, biology, and prognostic factors. Seminars in surgical oncology 2000;19(1):3-10.

5. Crispens MA. Borderline ovarian tumours: a review of the recent literature. Curr Opin Obstet Gynecol 2003;15(1):39-43.

6. Young RC, Walton LA, Ellenberg SS, et al. Adjuvant therapy in stage I and stage II epithelial ovarian cancer. Results of two prospective randomized trials. N Engl J Med 1990;322(15):1021-7.

7. Leitao MM, Jr., Boyd J, Hummer A, et al. Clinicopathologic analysis of early-stage sporadic ovarian carcinoma. Am J Surg Pathol 2004;28(2):147-59.

8. Sonis ST, Elting LS, Keefe D, et al. Perspectives on cancer therapy-induced mucosal injury: pathogenesis, measurement, epidemiology, and consequences for patients. Cancer 2004;100(9 Suppl):1995-2025.

9. Bell DA. Origins and molecular pathology of ovarian cancer. Mod Pathol 2005;18 Suppl 2:S19-32.

10. Shih le M, Kurman RJ. Ovarian tumorigenesis: a proposed model based on morphological and molecular genetic analysis. Am J Pathol 2004;164(5):1511-8.

11. Sieben NL, Macropoulos P, Roemen GM, et al. In ovarian neoplasms, BRAF, but not KRAS, mutations are restricted to low-grade serous tumours. J Pathol 2004;202(3):336-40.

12. Singer $\mathrm{G}$, Oldt $\mathrm{R}, 3 \mathrm{rd}$, Cohen $\mathrm{Y}$, et al. Mutations in BRAF and KRAS characterize the development of low-grade ovarian serous carcinoma. J Natl Cancer Inst 2003;95(6):484-6.

13. Salani R, Kurman RJ, Giuntoli R, 2nd, et al. Assessment of TP53 mutation using purified tissue samples of ovarian serous carcinomas reveals a higher mutation rate than previously reported and does not correlate with drug resistance. Int J Gynecol Cancer 2008;18(3):48791.

14. Shih le M, Kurman RJ. Molecular pathogenesis of ovarian borderline tumors: new insights and old challenges. Clin Cancer Res 2005;11(20):7273-9.

15. Bonome T, Lee JY, Park DC, et al. Expression profiling of serous low malignant potential, low-grade, and high-grade tumors of the ovary. Cancer research 2005;65(22):10602-12.

16. Meinhold-Heerlein I, Bauerschlag D, Hilpert F, et al. Molecular and prognostic distinction between serous ovarian carcinomas of varying grade and malignant potential. Oncogene 2005;24(6):1053-65.

17. Ouellet V, Provencher DM, Maugard CM, et al. Discrimination between serous low malignant potential and invasive epithelial ovarian tumors using molecular profiling. Oncogene 2005;24(29):4672-87.

18. Sieben NL, Oosting J, Flanagan AM, et al. Differential gene expression in ovarian tumors reveals Dusp 4 and Serpina 5 as key regulators for benign behavior of serous borderline tumors. J Clin Oncol 2005;23(29):7257-64.

19. Nowee ME, Snijders AM, Rockx DA, et al. DNA profiling of primary serous ovarian and fallopian tube carcinomas with array comparative genomic hybridization and multiplex ligation-dependent probe amplification. J Pathol 2007;213(1):46-55.

20. Weiss MM, Hermsen MA, Meijer GA, et al. Comparative genomic hybridisation. Mol Pathol 1999;52(5):243-51.

21. Schouten JP, McElgunn CJ, Waaijer R, Zwijnenburg D, Diepvens F, Pals G. Relative quantification of 40 nucleic acid sequences by multiplex ligation-dependent probe amplification. Nucleic acids research 2002;30(12):e57.

22. Coffa J, van de Wiel MA, Diosdado B, Carvalho B, Schouten J, Meijer GA. MLPAnalyzer: data analysis tool for reliable automated normalization of MLPA fragment data. Cell Oncol 2008;30(4):323-35.

23. Ihaka. R: A language for data analysis and graphics. J Comput Graphical Stat 1996;5(3):299314. 
24. Zakut H, Ehrlich G, Ayalon A, et al. Acetylcholinesterase and butyrylcholinesterase genes coamplify in primary ovarian carcinomas. The Journal of clinical investigation 1990;86(3):900-8.

25. Taira T, Maeda J, Onishi T, et al. AMY-1, a novel C-MYC binding protein that stimulates transcription activity of C-MYC. Genes Cells 1998;3(8):549-65.

26. Jauliac S, Lopez-Rodriguez C, Shaw LM, Brown LF, Rao A, Toker A. The role of NFAT transcription factors in integrin-mediated carcinoma invasion. Nature cell biology 2002;4(7):540-4.

27. Sutherland KD, Lindeman GJ, Choong DY, et al. Differential hypermethylation of SOCS genes in ovarian and breast carcinomas. Oncogene 2004;23(46):7726-33.

28. Mayr D, Kanitz V, Anderegg B, et al. Analysis of gene amplification and prognostic markers in ovarian cancer using comparative genomic hybridization for microarrays and immunohistochemical analysis for tissue microarrays. American journal of clinical pathology 2006;126(1):101-9.

29. Camilleri-Broet S, Hardy-Bessard AC, Le Tourneau A, et al. HER-2 overexpression is an independent marker of poor prognosis of advanced primary ovarian carcinoma: a multicenter study of the GINECO group. Ann Oncol 2004;15(1):104-12.

30. Hogdall EV, Christensen L, Kjaer SK, et al. Distribution of HER-2 overexpression in ovarian carcinoma tissue and its prognostic value in patients with ovarian carcinoma: from the Danish MALOVA Ovarian Cancer Study. Cancer 2003;98(1):66-73.

31. Nakayama K, Nakayama N, Kurman RJ, et al. Sequence mutations and amplification of PIK3CA and AKT2 genes in purified ovarian serous neoplasms. Cancer Biol Ther 2006;5(7):779-85.

32. Bautista $\mathrm{S}$, Valles $\mathrm{H}$, Walker RL, et al. In breast cancer, amplification of the steroid receptor coactivator gene AIB1 is correlated with estrogen and progesterone receptor positivity. Clin Cancer Res 1998;4(12):2925-9.

33. Osterberg L, Levan K, Partheen K, et al. Potential predictive markers of chemotherapy resistance in stage III ovarian serous carcinomas. BMC cancer 2009;18(9):368-79.

34. Sunde JS, Donninger $\mathrm{H}, \mathrm{Wu} \mathrm{K}$, et al. Expression profiling identifies altered expression of genes that contribute to the inhibition of transforming growth factor-beta signaling in ovarian cancer. Cancer research 2006;66(17):8404-12.

35. Tanner MM, Grenman S, Koul A, et al. Frequent amplification of chromosomal region 20q12-q13 in ovarian cancer. Clin Cancer Res 2000;6(5):1833-9.

36. Yamamoto $\mathrm{S}$, Tsuda $\mathrm{H}$, Honda $\mathrm{K}$, et al. Actinin-4 gene amplification in ovarian cancer: a candidate oncogene associated with poor patient prognosis and tumor chemoresistance. Mod Pathol 2009;22(4):499-507.

37. Vang R, Shih le M, Kurman RJ. Ovarian low-grade and high-grade serous carcinoma: pathogenesis, clinicopathologic and molecular biologic features, and diagnostic problems. Advances in anatomic pathology 2009;16(5):267-82.

38. Taylor CR. The WHO classification of lymphomas: cost-effective immunohistochemistry using a deductive reasoning "decision tree" approach: part II: the decision tree approach: diffuse patterns of proliferation in lymph nodes. Appl Immunohistochem Mol Morphol 2009;17(6):470-82.

39. John T, Liu G, Tsao MS. Overview of molecular testing in non-small-cell lung cancer: mutational analysis, gene copy number, protein expression and other biomarkers of EGFR for the prediction of response to tyrosine kinase inhibitors. Oncogene 2009;28 Suppl 1:S1423.

40. Matsugi T, Morishita K, Ihle JN. Identification, nuclear localization, and DNA-binding activity of the zinc finger protein encoded by the Evi-1 myeloid transforming gene. Molecular and cellular biology 1990;10(3):1259-64.

41. Nanjundan M, Nakayama Y, Cheng KW, et al. Amplification of MDS1/EVI1 and EVI1, located in the 3q26.2 amplicon, is associated with favorable patient prognosis in ovarian cancer. Cancer Res 2007;67(7):3074-84.

42. Quinn MC, Filali-Mouhim A, Provencher DM, Mes-Masson AM, Tonin PN. Reprogramming of the transcriptome in a novel chromosome 3 transfer tumor suppressor ovarian cancer 
cell line model affected molecular networks that are characteristic of ovarian cancer. Molecular carcinogenesis 2009;48(7):648-61.

43. Davila M, Frost AR, Grizzle WE, Chakrabarti R. LIM kinase 1 is essential for the invasive growth of prostate epithelial cells: implications in prostate cancer. The Journal of biological chemistry 2003;278(38):36868-75.

44. Smith HW, Marshall CJ. Regulation of cell signalling by uPAR. Nature reviews;11(1):23-36.

45. Bagheri-Yarmand R, Mazumdar A, Sahin AA, Kumar R. LIM kinase 1 increases tumor metastasis of human breast cancer cells via regulation of the urokinase-type plasminogen activator system. Int J Cancer 2006;118(11):2703-10.

46. Schmalfeldt B, Prechtel D, Harting $\mathrm{K}$, et al. Increased expression of matrix metalloproteinases (MMP)-2, MMP-9, and the urokinase-type plasminogen activator is associated with progression from benign to advanced ovarian cancer. Clin Cancer Res 2001;7(8):2396-404.

47. Rayet B, Gelinas C. Aberrant rel/nfkb genes and activity in human cancer. Oncogene 1999;18(49):6938-47.

48. Baldwin AS, Jr. The NF-kappa B and I kappa B proteins: new discoveries and insights. Annual review of immunology 1996;14:649-83.

49. Hayashida $\mathrm{Y}$, Honda $\mathrm{K}$, Idogawa $\mathrm{M}$, et al. E-cadherin regulates the association between beta-catenin and actinin-4. Cancer research 2005;65(19):8836-45.

50. Barbolina MV, Adley BP, Kelly DL, et al. Motility-related actinin alpha-4 is associated with advanced and metastatic ovarian carcinoma. Laboratory investigation; a journal of technical methods and pathology 2008;88(6):602-14.

51. Shibasaki F, Fukami K, Fukui Y, Takenawa T. Phosphatidylinositol 3-kinase binds to alphaactinin through the p85 subunit. The Biochemical journal 1994;302 ( Pt 2):551-7.

52. Ding Z, Liang J, Lu Y, et al. A retrovirus-based protein complementation assay screen reveals functional AKT1-binding partners. Proceedings of the National Academy of Sciences of the United States of America 2006;103(41):15014-9.

53. Yamamoto $\mathrm{S}$, Tsuda $\mathrm{H}$, Honda $\mathrm{K}$, et al. Actinin-4 expression in ovarian cancer: a novel prognostic indicator independent of clinical stage and histological type. Mod Pathol 2007;20(12):1278-85.

54. Gojis O, Rudraraju B, Alifrangis C, Krell J, Libalova P, Palmieri C. The role of steroid receptor coactivator-3 (SRC-3) in human malignant disease. Eur J Surg Oncol;36(3):224-9.

55. Mussi P, Yu C, O'Malley BW, Xu J. Stimulation of steroid receptor coactivator-3 (SRC-3) gene overexpression by a positive regulatory loop of E2F1 and SRC-3. Molecular endocrinology (Baltimore, Md 2006;20(12):3105-19.

56. De Meyer T, Bijsmans I, Van de Vijver K, et al. E2Fs mediate a fundamental cell-cycle deregulation in high-grade serous ovarian carcinomas. J Pathol 2008;217(1):14-20.

57. Qin L, Liao L, Redmond A, et al. The AIB1 oncogene promotes breast cancer metastasis by activation of PEA3-mediated matrix metalloproteinase 2 (MMP2) and MMP9 expression. Molecular and cellular biology 2008;28(19):5937-50.

58. Campbell IG, Russell SE, Phillips WA. PIK3CA mutations in ovarian cancer. Clin Cancer Res 2005;11(19 Pt 1):7042; author reply -3.

59. Skomedal H, Kristensen GB, Abeler VM, Borresen-Dale AL, Trope C, Holm R. TP53 protein accumulation and gene mutation in relation to overexpression of MDM2 protein in ovarian borderline tumours and stage I carcinomas. J Pathol 1997;181(2):158-65. 


\section{CHAPTER 5}

\section{Identification of candidate tumor suppressor genes in ovarian serous borderline tumors and carcinomas: an epigenetic approach}

Ingrid T.G.W. Bijsmans, Debbie G.J. Robbrecht, Leander Van Neste, Joo-Mi Yi, Kornel E. Schuebel, Maté Ongenaert, Wim Van Criekinge, Koen K. Van de Vijver, Adriaan P. de Bruïne, Nathalie L.G. Sieben and Manon van Engeland

In preparation 


\begin{abstract}
Serous ovarian tumors can be divided into serous borderline tumors (SBTs) and carcinomas (SCAs). The most important discriminators between these tumor entities are the presence of stromal invasion, prognosis, and genetic alterations such as $B R A F$, KRAS or TP53 mutations. Only a few studies investigating epigenetic differences between SBTs and SCAs have been published. The aim of this study was to identify promoter CpG island inactivated tumor suppressor genes (TSGs) and to study whether these methylation markers can discriminate between SBTs and SCAs. Identification of methylation markers was performed using three different approaches: 1) a candidate gene approach (genes reported to be hypermethylated in other tumor types); 2) a bioinformatics approach based on the sequence characteristics of promoter CpG islands; and 3) pharmacological unmasking of hypermethylated TSGs by treating ovarian cancer cell lines with the demethylating agent 5-aza-2'-deoxycytidine. Overall, our results indicate that methylation of the identified and selected candidate markers is not a frequently occurring event in serous ovarian tumors. The pharmacological unmasking approach identified BHMT2 as a novel methylation marker in serous ovarian tumors, methylated in $48 / 64(75.0 \%)$ of SCAs, while statistically significant less methylated in SBTs (16/32 (50.0\%), $p=0.014)$.
\end{abstract}




\section{Introduction}

Epithelial ovarian cancer is a leading cause of death in women suffering from cancer of the female genital tract. This disease is usually diagnosed at an advanced stage, because obvious symptoms at early stages are absent and in most cases the tumor has spread to the upper abdomen at time of diagnosis $[1,2]$. Worldwide, ovarian cancer was estimated to account for 225,000 cases and 140,000 deaths in 2008 [3].

Serous carcinomas (SCAs) are the most common ovarian tumors. Serous borderline tumors (SBTs) belong to an intermediate group of ovarian tumors and are characterized by stratified epithelial cells, varying nuclear atypia, and lack of destructive stromal invasion [2, 4]. SBT patients may present with tumor deposits, socalled implants. Nevertheless, the 5-year survival remains excellent (90-95\%). The most important discriminator between SBTs and SCAs is the presence of stromal invasion [2]. Long-term survival rates among patients with early-stage SCA (FIGO stage I/II) range from 80 to $90 \%$, whereas the 5-year survival rate of advanced-stage SCA (stage III/IV) is only $25 \%$. From a clinical and prognostic point of view the histological differentiation between SBTs and SCAs is important, since both patient management and prognosis differ substantially. Therefore, it is essential that the pathological classification of these tumors is correct. Previous studies showed that review of earlystage ovarian carcinomas by referring pathologists resulted in reclassification of up to $33 \%$ of the carcinomas as borderline tumors $[5,6]$. As a result, SBT patients are at risk of overtreatment. An objective tool to assess the differential diagnosis can assist pathologists in making the decision. The question whether SBTs are precursors of SCAs or represent different entities has been studied extensively. We and others showed that mutations in either BRAF or KRAS occur in approximately $60 \%$ of SBTs, while BRAF mutations have not been identified in SCAs. KRAS mutations have been detected in up to $12 \%$ of SCAs $[7,8]$. The tumor suppressor gene (TSG) TP53 is mutated in up to $80 \%$ of SCAs, while the prevalence is very low (0-8\%) in SBTs $[2,9,10]$. These genetic alterations indicate that SBTs and SCAs are different entities. Promoter CpG island hypermethylation, an epigenetic mechanism to inactivate TSGs [11], has been well studied in colorectal, breast and lung cancer. However, limited data are available for SBTs and SCAs, since the majority of epigenetic studies in ovarian cancer analyze all histological subtypes simultaneously and do not discriminate between them (e.g. [1215]). Basic knowledge on the role of epigenetics in ovarian cancer is of importance, especially since ovarian cancer is one of the first solid tumors for which epigenetic therapy using the demethylating agents azacytidine or decitabine is being proposed $[16,17]$. In the current study, promoter CpG island hypermethylation was analyzed in serous ovarian tumors to identify genes able to discriminate between SBTs and SCAs. Three approaches were performed to identify novel methylation markers: 1) a candidate gene approach in which genes were selected from literature reported to be hypermethylated in other tumor types; 2) a recently published bioinformatics approach making use of sequence motifs in the promoter region [18]; and 3) pharmacological unmasking of hypermethylated TSGs in ovarian cancer cell lines as described previously [19]. 


\section{Materials and methods}

\section{Cell lines and patient material}

Ovarian cancer cell lines SKOV3 and OVCAR3 were purchased from ATCC. COV318, COV413A, and COV504 were kindly provided by Dr. J van Eendenburg (Leiden University Medical Center (LUMC), Leiden, The Netherlands). All cell lines were maintained in RPMI-1640 medium (Gibco, Invitrogen) supplemented with $10 \%$ fetal calf serum (FCS; Gibco) and 1\% glutamax (Gibco). Cell line OV90 (ATCC) was cultured in complete growth medium (1:1 mixture of medium 199 and MCDB105 (Sigma-Aldrich, St. Louis, USA)), supplemented with $10 \%$ FCS and $1 \%$ glutamax. Fresh-frozen or formalin-fixed paraffin-embedded tissue of 23 fallopian tubes (FTs), 32 SBTs and 64 SCAs (Silverberg grade 1, well differentiated (SCA1s), $n=8$; grade 2, moderately differentiated (SCA2s), $n=18$; and grade 3, poorly differentiated (SCA3s), $n=38$ ) was obtained from the Maastricht Pathology Tissue Collection (MPTC; Maastricht University Medical Center, MUMC), the archive of the Department of Pathology of LUMC, and Stichting PAMM (Eindhoven, The Netherlands). Diagnoses were confirmed by experienced gynecologic pathologists (NS, KVdV) by reviewing $4 \mu \mathrm{m}$ haematoxylin and eosin stained sections. See Table 1 for detailed clinicopathological characteristics. Baseline characteristics (International Federation of Gynecology and Obstetrics (FIGO) stage, age at diagnosis, cancer related death and overall mortality) of the samples derived from the different hospitals did not differ significantly, therefore, the patient groups were considered one group (data not shown). None of the patients received chemotherapy prior to surgery. Histologically normal FTs derived from non-cancerous patients were used as normal control. Collection, storage and use of tissue and patient data were performed in agreement with the "Code for Proper Secondary Use of Human Tissue in The Netherlands" (http://www.fmwv.nl). This study was approved by the Medical Ethical Committee of the MUMC.

Table 1: Clinicopathological characteristics

\begin{tabular}{lll}
\hline Characteristics & & Patients (n=119) \\
\hline & FT & n (\%) \\
& SBT & $32(19.3)$ \\
& SCA & $64(53.8)$ \\
& & \\
Age at diagnosis & & $31.3(20-40)$ \\
Years (range) & FT & $46.1(19-77)$ \\
& SBT & $60.7(21-87)$ \\
& SCA & \\
FIGO stage* & & $19(59.4)$ \\
SBT & & $1(3.1)$ \\
& stage I & $11(34.4)$ \\
SCA & stage II & $10(15.6)$ \\
& stage III & $5(7.8)$ \\
& stage I & $45(70.3)$ \\
& stage II & $3(4.7)$ \\
\hline${ }^{*}$ Age and FIGO stage were unavailable for 2 patients (1 SBT, 1 SCA)
\end{tabular}


DAC and TSA treatment of ovarian cancer cell lines

The cell lines COV318, COV504, OVCAR3 and SKOV3 were treated with 5-aza-2'deoxycytidine (DAC) and Trichostatin A (TSA). To inhibit DNA methylation, ovarian cancer cells at $10 \%$ confluency were treated with $5 \mu \mathrm{M}$ 5-aza-2'-deoxycytidine (SigmaAldrich; $1 \mathrm{mM}$ stock solution in PBS) for 96 hours, replacing DAC-containing medium every 24 hours. Treatment with 300 nM TSA (Sigma-Aldrich; 1.5 mM stock solution in ethanol) to prevent histone deacetylation, was performed on $30 \%$ confluent cells for 18 hours. In parallel, control cells (mock) were treated with equal volumes of PBS or ethanol without drugs respectively.

\section{DNA isolation}

Tumor tissue from ten haematoxylin-stained $10 \mu \mathrm{m}$ sections was microdissected with a 10G needle under transmitted light microscopy. The tumor sections contained at least $75 \%$ tumor cells. DNA was extracted using Puregene DNA isolation kit (Gentra Systems, BIOzymTC; Landgraaf, The Netherlands) according to manufacturer's description.

\section{Pharmacologic microarray analysis}

Microarray analysis was performed on DAC, TSA and mock treated cell lines COV318, COV504, OVCAR3 and SKOV3, as described by Schuebel et al. [19]. Briefly, total RNA of mock, DAC, and TSA treated cell lines OVCAR3 and SKOV3 was isolated using Trizol (Invitrogen), for COV318 and COV504 we used the RNeasy kit (Qiagen). RNA quantity and quality were determined using the Nanodrop ND-100 and 2100 Bioanalyzer (Agilent Technologies) respectively. Sample amplification and labeling were performed using the Low RNA Input Fluorescent Linear Amplification Kit (Agilent Technologies), followed by purification with RNeasy mini kit (Qiagen) and quantification. Cy3 or Cy5 labeled RNA samples (750 ng) were mixed with control targets (Agilent Technologies) followed by hybridization on $4 \times 44 \mathrm{~K}$ whole genome microarrays (Agilent Technologies) and processed according to the manufacturer's protocol. Scanning was done with the Agilent G2565BA microarray scanner using Agilent's recommended settings. Array data were analyzed using $\mathrm{R}$ and BioConductor, using the limma package [20]. Median Cy5 and Cy3 signals were read from the raw data and M-values, i.e. $\log 2$ (Cy5/Cy3), were loess normalized. Basal expression was roughly estimated using the single channel Cy3 values, containing the mock treated samples. Based on the threshold used for basal expression, the specificity could be further increased, with obvious implications on sensitivity.

\section{Candidate TSG selection}

To identify candidate TSGs silenced by promoter CpG island hypermethylation in serous ovarian cancer, we used three approaches (Figure 1). In the first candidate gene approach we analyzed the genes APC, BRCA1, CHFR, GATA4, GATA5, HLTF, O 6 -MGMT, hMLH1, $p 14^{A R F}, p 16^{I N K 4 A}$, RASSF2, RUNX3, SFRP1/2/4/5, and WIF1 selected from literature reported to be frequently and functionally hypermethylated in multiple tumor types [21] and FANCF was chosen because previous studies showed promoter $\mathrm{CpG}$ island hypermethylation in ovarian cancer. 


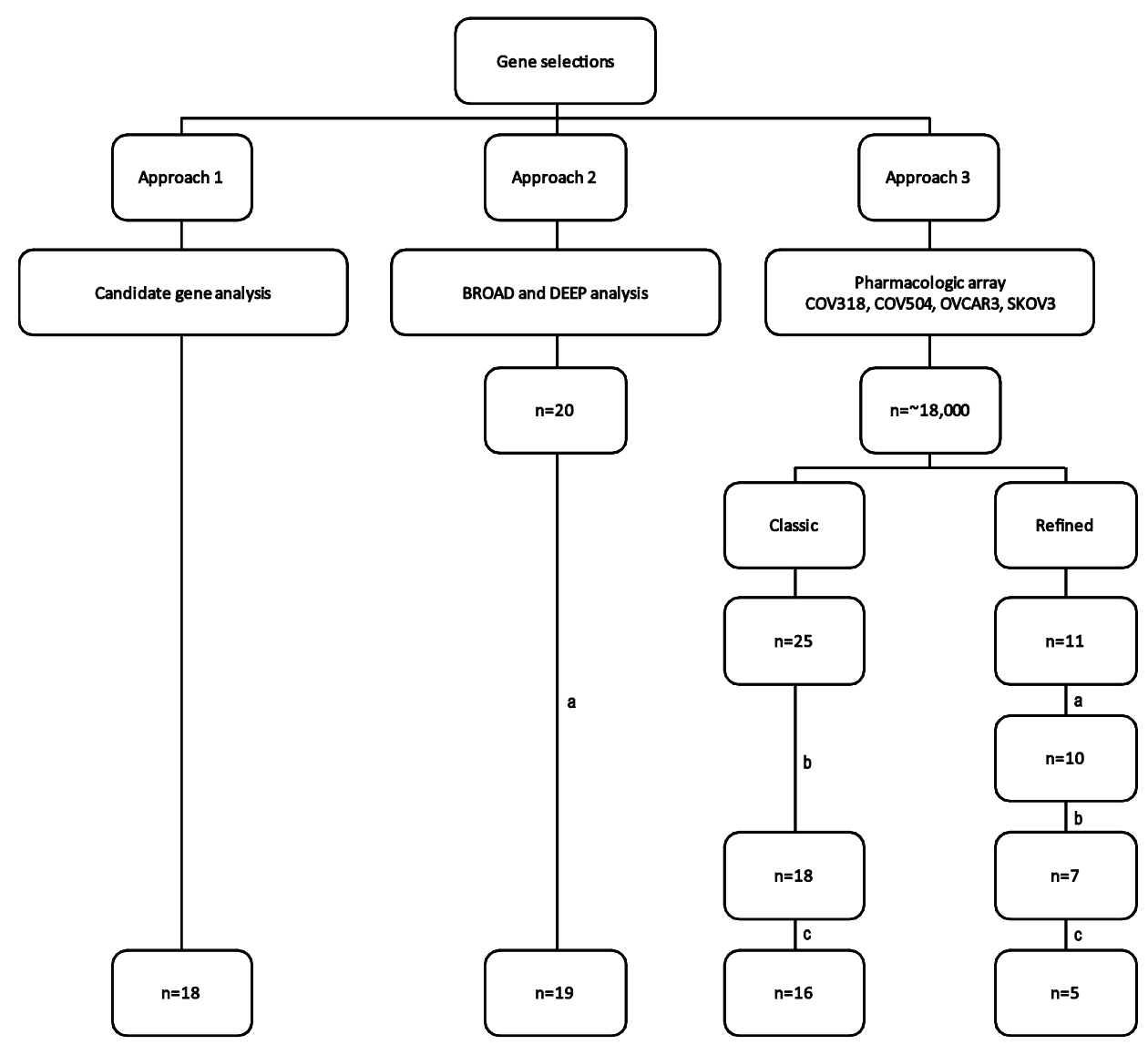

Figure 1: Flowchart showing candidate TSG selection

Identification of novel hypermethylated genes was performed using the approaches 1 to 3 : candidate gene analysis, BROAD and DEEP analyses or pharmacologic array respectively. a: elimination of genes without a CpG island; $b$ : methylation assessed in ovarian cancer cell lines. Elimination if less than 2 cell lines showed methylation; c: methylation assessed in normal tissue. Candidate was eliminated if more than $30 \%$ of FTs showed methylation. The remaining candidate genes were analyzed in SBTs and SCAs.

The second strategy involved BROAD and DEEP analyses [18] that was integrated with differentially expressed genes derived from mRNA expression microarray data of SBTs and SCAs that we have published previously [22]. Briefly, BROAD analysis predicts whether a promoter CPG island is prone to methylation in cancer and is based on genome-wide promoter alignments of methylated CpG islands. Genome-wide clustal alignment of promoter sequences is matched to promoter sequences of 56 reported cancer-specific methylated genes. Genes with a given minimal homology to these 56 known cancer-specific methylated genes were selected for analysis. DEEP analysis makes use of seven sequence motifs (GGGC*GC*C, GCC*GCAC, CTGGG*GA, CCC**GCGCC, AGCTG**CT, A*GGC*GGG, and $\left.A^{*} C G C^{*} G C C\right)$ in a symmetrical region of $1 \mathrm{~kb}$ around the predicted transcription start site (TSS) which have been identified to be commonly present in $\mathrm{CpG}$ island-containing promoter regions to discriminate 
between cancer-specific methylated genes and genes that are not methylated in normal tissue. Genes were selected if four or more of these sequence motifs were present. Overlapping BROAD and DEEP identified candidate genes with a list of genes differentially expressed between SBT and SCAs previously identified through expression microarray [22] generated a list of 20 genes (ACTN1, BARD1, C11orf2, DBF4, DEK, FANCG, HDGF, HPSE, INPP4B, MYBL2, NDC80, NDP, PLLP, POLR3D, SMPD2, TK1, TNFRSF1OB, TPPP3, TRIP13, and TTK). The last approach involved pharmacological unmasking of hypermethylated TSGs in ovarian cancer cell lines as described above. Selection criteria for gene identification were: 1) basal expression was low/absent in wild-type ovarian cancer cell lines; 2) re-expression was detected after DAC treatment; while 3) TSA response was absent, i.e. not discernable from background and down in expression array. The DAC-responsive zone contains two tier regions: top tier and next tier (Figure 2). The top tier (TT) is defined as a zone in which gene expression increased at least 2-fold after DAC treatment, while unaffected by TSA treatment. The next tier (NT) contains candidate genes of which DAC treatment increased gene expression between 1.41 and 2-fold and TSA treatment did not increase or decrease gene expression.
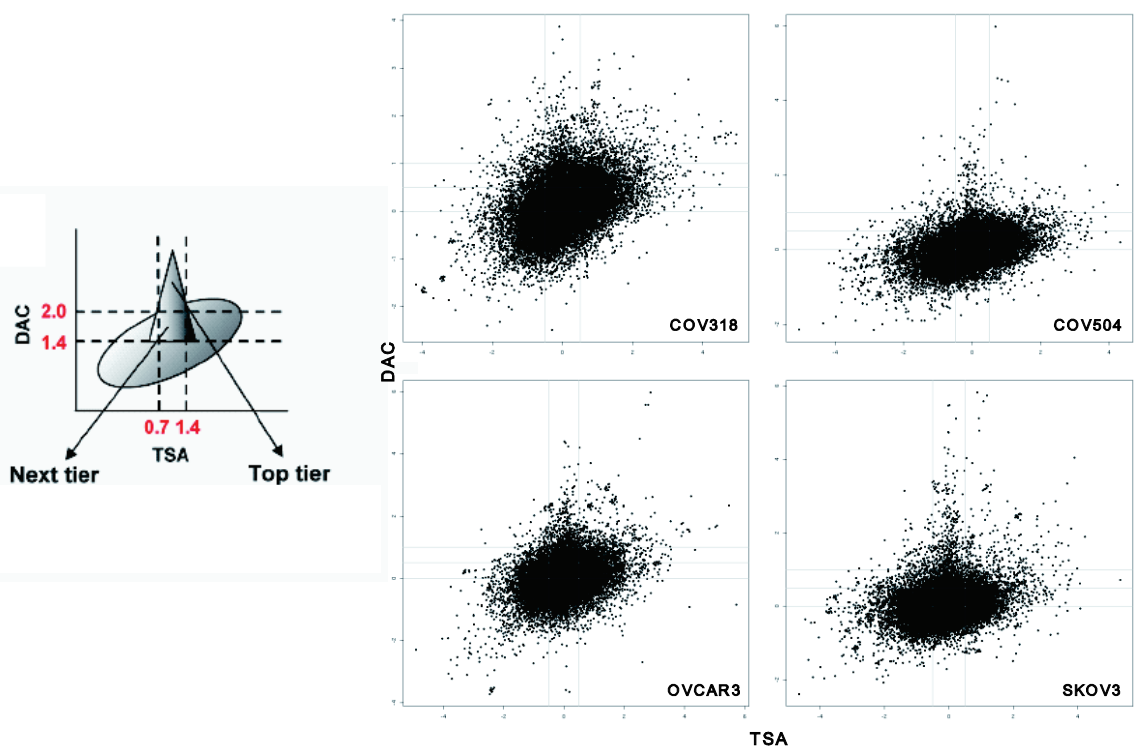

Figure 2: Illustration of gene-expression changes in DAC and TSA treated ovarian cancer cell lines Schematic representation of top tier (DAC treatment increased expression $\geq 2$-fold, TSA had no effect on expression, and wild-type (WT) cells showed no basal expression) and next tier (expression increased between 1.41 and 2-fold after DAC treatment, TSA and WT same as top tier) is shown in the left panel; adapted from Schuebel et al., PLoS Genet. 2007;3(9):1709-23. The right panel represents the scatter-plots of the cell lines COV318, COV504, OVCAR3 and SKOV3. 
In a first attempt to identify candidate genes using this approach, we used the procedure as described by Schuebel et al. [19], named "classic approach". Genes present in the TTs and NTs of one or more ovarian cancer cell lines and differentially expressed between SBT and SCAs as previously identified through expression microarray [22] were selected based on gene ontology terms. The selected genes included B3GALT4, B3GNT3, CACNG6, CD44, CLDN6, CST6, DUSP2, DUSP6, FLNC, FN1, FNDC1, FOXF2, ICAM1, IL6R, IRS1, MAPK13, ME3, OSMR, PIGC, POMC, PROS1, PYCARD, RBP1, SPINT1, and TNFRSF8. Later, the stratification of expression levels was further refined to reduce the level of false positive candidates, thereby enabling more specificity. This approach was termed "refined approach", based on the findings described by McGarvey et al. [23]. The upper margin of the silent expression range was chosen so that $90 \%$ of the verified hypermethylated genes derived from the classic approach were present within this zone. This cut-off was also chosen because the general expression trends of the verified genes were separated from a set of falsepositive genes [19], thereby increasing the probability of a candidate gene. As an additional inclusion criterion the gene had to be present in the TT of at least two ovarian cancer cell lines and differential expression between SBTs and SCAs according to previously generated expression profiling [22] leading to the selection of the genes ANK1, BHMT2, CDO1, CYP26A1, GJB3, GPER, GPR68, LOXL4, MATN2, MFI2, and VAMP5. More than $35 \%$ methylation was defined as frequently methylated.

\section{Sodium bisulfite treatment and methylation specific PCR (MSP)}

Sodium bisulfite treatment was performed on 500 ng genomic DNA using the EZ DNA methylation $\mathrm{Kit}^{\mathrm{TM}}$ (Zymo research, Orange, CA, USA), according to manufacturer's description. Determination of the TSS was performed using DBTSS (http://dbtss.hgc.jp), CpGPlot (http://www.ebi.ac.uk/Tools/emboss/cpgplot/index. html) was used for $\mathrm{CpG}$ island confirmation using default settings (length $\mathrm{CpG}$ island $>200$ base pairs, GC content $>50 \%$ ). MSP analysis was performed by a nested approach in 96-well PCR plates (Greiner Bio-One, Alphen a/d Rijn, The Netherlands), as reported previously [24-26]. MSP primer sequences and PCR conditions are available on request.

\section{Data analysis}

Associations between patient characteristics of the different hospitals, and the comparison of methylation frequencies between SBTs and SCAs were calculated using Pearson Chi-Square test or Independent-Samples T Test where appropriate. All $p$ values are two-sided and $\mathrm{p} \leq 0.05$ was considered statistically significant. Analyses were done using SPSS 15.0 for Windows. 


\section{Results}

Methylation analysis of literature-based candidate genes

We detected a statistically significant difference in methylation frequency, as measured by MSP, between SBTs and SCAs (Table 2) for APC (11/32 (3.1\%) and 15/63 $(23.8 \%)$ respectively; $\mathrm{p}=0.011)$. Because the $A P C$ methylation frequency was below $35 \%$ this gene was not further analyzed. None of the FTs, SBTs, or SCAs showed CHFR methylation. For ten genes (FANCF, GATA4, GATA5, HLTF, RASSF2, RUNX3, SFRP1/2/4/5), the methylation frequencies were below $15 \%$ in both SBTs and SCAs and therefore not studied further. Higher methylation frequencies in SBTs and SCAs were detected for BRCA1 (11/32 (34.4\%) and 23/63 (36.5\%)), p14 ${ }^{A R F}(8 / 32$ (25.0\%) and 14/63 $(22.2 \%))$, and $p 16^{I N K 4 A}(7 / 31(22.6 \%)$ and $10 / 61(16.4 \%))$. However, methylation of these CpG islands was also detected frequently in FTs (9/21 (42.9\%), 6/20 (30.0\%) and $4 / 20(20.0 \%)$ respectively), indicating tissue-specific methylation, and not cancerspecific methylation for the region analyzed by the MSP primers and therefore not suitable as biomarkers for discriminating SBTs from SCAs.

\section{$B R O A D$ and DEEP analysis}

A novel approach to identify candidate methylation markers based on genes differentially expressed in SBTs and SCAs combined with genome-wide promoter alignment and/or promoter sequence motifs was performed next. Genes identified by BROAD analysis include ACTN1, C11orf2, DBF4, FANCG, HPSE, NDC80, NDP, TK1, TNFRSF1OB, and TPPP3. BARD1, MYBL2, POLR3D, and TTK were identified by DEEP analysis. DEK, HDGF, PLLP, SMPD2, and TRIP13 fulfilled both BROAD and DEEP selection criteria. The genes C11orf2, DBF4, FANCG, HDGF, PLLP, POLR3D, TK1, TPPP3, and TRIP13 showed methylation frequencies below 15\% (Table 2). HPSE and TTK did not show methylation in FTs, SBTs or SCAs. TNFRSF1OB promoter CpG island hypermethylation was detected in $12 / 32$ (37.5\%) of SBTs and $31 / 63$ (49.2\%) of SCAs. Even though the methylation frequency at the examined CpG loci is high, this gene cannot be used as a novel ovarian cancer methylation marker in the discrimination between SBTs and SCAs since methylation frequencies did not differ $(p=0.279)$.

Pharmacological unmasking of hypermethylated TSGs identified novel candidate genes Gene expression in the ovarian cancer cell lines COV318, COV504, OVCAR3, and SKOV3 upon DAC or TSA treatment was analyzed by microarray analysis. The "classic approach" as described in detail previously [19] was performed. Twenty-five genes (Figure 1 and Table 2) were selected based on gene ontology terms. Promoter CpG island hypermethylation analysis of candidate methylation markers was assessed by MSP in the ovarian cancer cell lines for validation. Of these 25 genes, 9, 1, 5 and 12 genes respectively were present in the TT; and 1, 0, 7 and 1 genes in the NT of COV318, COV504, OVCAR3, and SKOV3 respectively. Table 3 shows that the frequency of validation of the examined regions varies largely between the cell lines: $1 / 5(20.0 \%)$ TT genes was methylated in OVCAR3, whereas 10/12 (83.3\%) TT genes were confirmed in SKOV3. Of the NT genes, methylation was detected in only $6(24.0 \%), 6(24.0 \%), 9$ (36.0\%) and 19 (76.0\%) genes of COV318, COV504, OVCAR3 and SKOV3 respectively. CD44, DUSP6, FN1, FOXF2, ICAM1, OSMR, and PIGC were excluded from further 
analysis because methylation was detected in only one cell line. The remaining 18 genes were tested for methylation in FTs. IRS1 and MAPK13 showed methylation in $14 / 21(66.7 \%)$ and $8 / 21(38.1 \%)$ of FTs respectively and were therefore not further analyzed, leaving 16 genes for methylation analysis in SBTs and SCAs. The methylation frequencies in SBTs and SCAs were below $15 \%$ for the majority of genes (B3GALT4, B3GNT3, CACNG6, CST6, DUSP2, FLNC, POMC, PROS1, PYCARD, RBP1, SPINT1, and TNFRSF8). Remarkably, methylation frequency for IL6R was $11 / 29$ (38.0\%) in SBTs and $3 / 63(4.8 \%)$ in SCAs $(p<0.001)$.

Table 2: Methylation frequencies in ovarian cancer cell lines, FTs, SBTs and SCAs

\begin{tabular}{|c|c|c|c|c|c|c|}
\hline Analysis & Gene & $\begin{array}{l}\text { Cell lines } \\
n=6\end{array}$ & $\begin{array}{l}\text { FT (\%) } \\
n=23\end{array}$ & $\begin{array}{l}\text { SBT (\%) } \\
n=32\end{array}$ & $\begin{array}{l}\text { SCA (\%) } \\
n=64\end{array}$ & $\begin{array}{l}\text { p- } \\
\text { value* }\end{array}$ \\
\hline \multirow{18}{*}{$\begin{array}{l}\text { Candidate gene } \\
\text { analysis }\end{array}$} & $A P C$ & ND & $1 / 23(4.3)$ & $1 / 32(3.1)$ & $15 / 63(23.8)$ & 0.011 \\
\hline & $B R C A 1$ & ND & $9 / 21(42.9)$ & $11 / 32(34.4)$ & $23 / 63(36.5)$ & NS \\
\hline & CHFR & ND & $0 / 23$ & $0 / 29$ & $0 / 60$ & \\
\hline & FANCF & ND & $0 / 23$ & $0 / 28$ & $1 / 63(1.6)$ & NS \\
\hline & GATA4 & ND & $2 / 23(8.7)$ & $4 / 30(13.3)$ & $4 / 62(6.5)$ & NS \\
\hline & GATA5 & ND & $0 / 23$ & $0 / 29$ & $3 / 61(4.9)$ & NS \\
\hline & HLTF & ND & $0 / 23$ & $0 / 32$ & $2 / 62(3.2)$ & NS \\
\hline & $0^{6}-M G M T$ & ND & $4 / 21(19.0)$ & $3 / 31$ (9.7) & $14 / 64(21.9)$ & NS \\
\hline & $h M L H 1$ & ND & $1 / 23(4.3)$ & $5 / 31(16.1)$ & $6 / 64(9.4)$ & NS \\
\hline & p14ARF & ND & $6 / 20(30.0)$ & $8 / 32(25.0)$ & $14 / 63(22.2)$ & NS \\
\hline & p16 INK4A & ND & $4 / 20(20.0)$ & $7 / 31(22.6)$ & 10/61 (16.4) & NS \\
\hline & RASSF2 & ND & $0 / 23$ & $1 / 27(3.7)$ & $1 / 61(1.6)$ & NS \\
\hline & RUNX3 & ND & $0 / 23$ & $4 / 30$ (13.3) & $6 / 62(9.7)$ & NS \\
\hline & SFRP1 & ND & $0 / 22$ & $0 / 32$ & $2 / 62$ (3.2) & NS \\
\hline & SFRP2 & ND & $0 / 23$ & $4 / 29(13.8)$ & $6 / 58$ (10.3) & NS \\
\hline & SFRP4 & ND & $4 / 23(17.4)$ & $4 / 31(12.9)$ & $4 / 62(6.5)$ & NS \\
\hline & SFRP5 & ND & $0 / 21$ & $1 / 27(3.7)$ & $4 / 60(6.7)$ & NS \\
\hline & WIF1 & ND & $4 / 23(17.4)$ & $11 / 31(35.5)$ & $19 / 58(32.8)$ & NS \\
\hline BROAD and & ACTN1 & ND & $2 / 22(9.1)$ & $8 / 32(25.0)$ & $22 / 62(35.5)$ & NS \\
\hline \multirow[t]{18}{*}{ DEEP analysis } & $B A R D 1$ & ND & $3 / 23(13.0)$ & $3 / 32(9.4)$ & $10 / 62(16.1)$ & NS \\
\hline & C11orf2 & ND & $1 / 23(4.3)$ & $0 / 30$ & $0 / 63$ & NS \\
\hline & DBF4 & ND & $1 / 22(4.5)$ & $1 / 31(3.2)$ & $6 / 62(9.7)$ & NS \\
\hline & DEK & ND & $1 / 23(4.3)$ & $2 / 32(6.3)$ & $11 / 62(17.7)$ & NS \\
\hline & FANCG & ND & $0 / 21$ & $0 / 28$ & $5 / 61(8.2)$ & NS \\
\hline & $H D G F$ & ND & $0 / 22$ & $1 / 32(3.1)$ & $1 / 62(1.6)$ & NS \\
\hline & HPSE & ND & $0 / 22$ & $0 / 32$ & $0 / 62$ & \\
\hline & MYBL2 & ND & $5 / 23(21.7)$ & $5 / 31(16.1)$ & $6 / 64(9.4)$ & NS \\
\hline & NDC80 & ND & $2 / 23(8.7)^{\prime}$ & $7 / 31$ (22.6) & $13 / 63(20.6)$ & NS \\
\hline & $N D P$ & ND & $5 / 23(21.7)$ & $9 / 32(28.1)$ & $13 / 62(21.0)$ & NS \\
\hline & PLLP & ND & $0 / 23$ & $1 / 32(3.1)^{\prime}$ & $5 / 63(7.9)$ & NS \\
\hline & POLR3D & ND & $2 / 22(9.1)$ & $0 / 30$ & $1 / 62(1.6)$ & NS \\
\hline & SMPD2 & ND & $4 / 22(18.2)$ & $1 / 32(3.1)$ & $5 / 63(7.9)$ & NS \\
\hline & TK1 & ND & $1 / 21(4.8)$ & $4 / 32(12.5)$ & $5 / 61$ (8.2) & NS \\
\hline & TNFRSF10B & ND & $3 / 23(13.0)$ & $12 / 32(37.5)$ & $31 / 63(49.2)$ & NS \\
\hline & TPPP3 & ND & $1 / 21(4.8)$ & $2 / 32(6.3)$ & $5 / 61(8.2)$ & NS \\
\hline & TRIP13 & ND & $1 / 22(4.5)$ & $0 / 32$ & $3 / 62$ (4.8) & NS \\
\hline & TTK & ND & $0 / 23$ & $0 / 32$ & $0 / 62$ & \\
\hline
\end{tabular}


Table 2: continued

\begin{tabular}{|c|c|c|c|c|c|c|}
\hline Analysis & Gene & $\begin{array}{l}\text { Cell lines } \\
n=6\end{array}$ & $\begin{array}{l}\text { FT (\%) } \\
n=23\end{array}$ & $\begin{array}{l}\text { SBT (\%) } \\
n=32\end{array}$ & $\begin{array}{l}\text { SCA (\%) } \\
n=64\end{array}$ & $\begin{array}{l}\text { p- } \\
\text { value* }\end{array}$ \\
\hline \multirow{25}{*}{$\begin{array}{l}\text { Pharmacologic } \\
\text { array } \\
\text { "classic" }\end{array}$} & B3GALT4 & $3 / 6(50.0)$ & $4 / 21(19.0)$ & $0 / 31$ & $4 / 61(6.6)$ & NS \\
\hline & B3GNT3 & $4 / 6(66.7)$ & $1 / 19(5.3)^{\prime}$ & $0 / 22$ & $2 / 54(3.7)$ & NS \\
\hline & CACNG6 & $3 / 6(50.0)$ & $0 / 21$ & $0 / 28$ & $1 / 59(1.7)$ & NS \\
\hline & CD44 & $1 / 6(16.7)$ & ND & ND & ND & \\
\hline & CLDN6 & $4 / 6(66.7)$ & $0 / 21$ & 4/32 (12.5) & $10 / 60(16.7)$ & NS \\
\hline & CST6 & $5 / 6(83.3)$ & $0 / 22$ & $0 / 29$ & $2 / 61$ (3.3) & NS \\
\hline & DUSP2 & $3 / 6(50.0)$ & $3 / 21(14.2)$ & $2 / 32$ (6.3) & $5 / 64(7.8)$ & NS \\
\hline & DUSP6 & $1 / 6(16.7)$ & ND & ND & ND & \\
\hline & FLNC & $3 / 6(50.0)$ & $0 / 20$ & $4 / 32(12.5)$ & $0 / 63$ & 0.004 \\
\hline & FN1 & $0 / 6$ & ND & ND & ND & \\
\hline & FNDC1 & $4 / 6(66.7)$ & $3 / 19(15.8)$ & $4 / 25(16.0)$ & $16 / 56(28.6)$ & NS \\
\hline & FOXF2 & $1 / 6(16.7)$ & ND & ND & ND & \\
\hline & ICAM1 & $1 / 6(16.7)$ & ND & ND & ND & \\
\hline & IL6R & $2 / 6(33.3)$ & 2/21 (9.5) & $11 / 29(38.0)$ & $3 / 63(4.8)$ & $<0.001$ \\
\hline & IRS1 & $3 / 6(50.0)$ & $14 / 21(66.7)$ & ND & ND & \\
\hline & MAPK13\# & $3 / 6(50.0)$ & $8 / 21(38.1)$ & ND & ND & \\
\hline & ME3 & $3 / 6(50.0)$ & $3 / 23(13.0)$ & $9 / 32(28.1)$ & $18 / 64(28.1)$ & NS \\
\hline & OSMR & $1 / 6(16.7)$ & ND & ND & ND & \\
\hline & PIGC & $1 / 6(16.7)$ & ND & ND & ND & \\
\hline & POMC & $3 / 6(50.0)$ & $1 / 21(4.8)$ & $1 / 31(3.2)$ & $3 / 59(5.1)$ & NS \\
\hline & PROS1 & $2 / 6(33.3)$ & $1 / 21(4.8)$ & $0 / 30$ & $1 / 63(1.6)$ & NS \\
\hline & PYCARD & $3 / 6(50.0)$ & $0 / 22$ & $1 / 28(3.6)$ & $0 / 58$ & NS \\
\hline & $R B P 1$ & $4 / 6(66.7)$ & $2 / 22(9.1)$ & $3 / 32(9.4)$ & $5 / 64(7.8)$ & NS \\
\hline & SPINT1 & $2 / 6(33.3)$ & $0 / 21$ & $1 / 31(3.2)$ & $1 / 64(1.6)$ & NS \\
\hline & TNFRSF8 & $3 / 6(50.0)$ & $1 / 21(4.8)$ & $1 / 31(3.2)$ & $4 / 60(6.7)$ & NS \\
\hline \multirow{11}{*}{$\begin{array}{l}\text { Pharmacologic } \\
\text { array } \\
\text { "refined" }\end{array}$} & ANK1 & $6 / 6(100.0)$ & $17 / 19$ (89.5) & ND & ND & \\
\hline & BHMT2 & $6 / 6(100.0)$ & $5 / 21(23.8)$ & $16 / 32(50.0)$ & $48 / 64(75.0)$ & 0.014 \\
\hline & CD01 & $5 / 6(83.3)$ & $2 / 22(9.1)$ & $3 / 30(10.0)$ & $42 / 64(65.6)$ & $<0.001$ \\
\hline & CYP26A1 & $1 / 6(16.7)$ & ND & ND & ND & \\
\hline & GJB3 & $2 / 6(33.3)$ & $1 / 21(4.8)$ & 1/32 (3.1) & $5 / 64(7.8)$ & NS \\
\hline & GPER & $4 / 6(66.7)$ & $0 / 23$ & $9 / 30(30.0)$ & $15 / 64(23.4)$ & NS \\
\hline & GPR68 & $4 / 6(66.7)$ & $10 / 13(76.9)$ & ND & ND & \\
\hline & LOXL4 & $2 / 6(33.3)$ & $1 / 22(4.5)$ & $0 / 32$ & $3 / 64(4.7)$ & NS \\
\hline & MATN2 & $1 / 6(16.7)$ & ND & ND & ND & \\
\hline & $M F / 2$ & $1 / 6(16.7)$ & ND & ND & ND & \\
\hline & VAMP5 & $6 / 6(100.0)$ & $19 / 19(100.0)$ & ND & ND & \\
\hline
\end{tabular}

Abbreviations: FT: fallopian tube; SBT: serous borderline tumor; SCA: serous carcinoma; ND: not determined; NS: not significant

${ }^{*}$ P-value SBT vs SCA

\#Next tier genes; all other genes are top tier genes

To decrease the number of false positives, a more stringent "refined" approach was subsequently performed (Figure 1 and Table 2). The number of genes in TTs and NTs reduced when applying these criteria, thereby increasing the number of potential candidate genes (Table 3). Using the classic approach, the TT and NT of COV318 contained 303 and 831 genes respectively. These numbers reduced to 63 and 148 genes respectively using the refined approach. The number of genes in respectively TT and NT reduced from 123 and 391 to 27 and 66 (COV504), from 286 and 781 to 95 and 169 (OVCAR3), and from 301 and 567 to 85 and 169 (SKOV3). As shown in Table 3, the number of validated genes, thus genes present in the TT and actually methylated by MSP increased for the cell lines OVCAR3 (5/8 (62.5\%) versus $1 / 5(20.0 \%))$ and SKOV3 $(7 / 8(87.5 \%)$ versus $10 / 12(83.3 \%))$, whereas this decreased for COV318 (1/5 (20.0\%) 
versus $3 / 9(33.3 \%))$ and COV504 (1/3 (33.3\%) versus $1 / 1(100.0 \%))$. It has to be taken in account that the TT of COV504 using the classic approach contained only one gene. CYP26A1, MATN2 and MFI2 were excluded because methylation was detected in 1 out of 6 ovarian cancer cell lines. ANK1 and GPR68 were eliminated because these genes showed $>35 \%$ methylation in FTs (17/19 (89.5\%) and 10/13 (76.9\%) respectively), leaving BHMT2, CDO1, GJB3, GPER, and LOXL4 for MSP analysis on SBTs and SCAs. We detected a statistically significant difference in methylation frequency in SBTs and SCAs for BHMT2 (16/32 (50.0\%) and 48/64 (75.0\%); $p=0.014)$ and CDO1 (3/30 (10.0\%) and $42 / 64(65.6 \%)$ respectively; $p<0.001)$. In contrast to data presented above, BHMT2 and CDO1 were frequently as well as differentially methylated between SBTs and SCAs. Decision tree analysis of all genes examined for methylation in SBTs and SCAs showed that CDO1 and BHMT2 were the main discriminators; the other genes analyzed were not of additional value (data not shown).

Table 3: Number of genes in top and next tiers of ovarian cancer cell lines

\begin{tabular}{lllllll}
\hline Approach & Cell line & $\begin{array}{l}\text { Methylation of } \\
\text { TT genes (\%) }\end{array}$ & $\begin{array}{l}\text { Methylation of } \\
\text { NT genes (\%) }\end{array}$ & $\begin{array}{l}\text { Overall } \\
\text { methylation (\%) }\end{array}$ & $\begin{array}{l}\text { \# of genes } \\
\text { in TT }\end{array}$ & $\begin{array}{l}\text { \# of genes } \\
\text { in NT }\end{array}$ \\
\hline Classic & COV318 & $3 / 9(33.3)$ & $1 / 3(33.3)$ & $6 / 25(24.0)$ & 303 & 831 \\
$(\mathrm{n}=25)$ & COV504 & $1 / 1(100.0)$ & $0 / 1$ & $6 / 25(24.0)$ & 123 & 391 \\
& OVCAR3 & $1 / 5(20.0)$ & $2 / 7(28.6)$ & $9 / 25(36.0)$ & 286 & 781 \\
& SKOV3 & $10 / 12(83.3)$ & $1 / 1(100.0)$ & $19 / 25(76.0)$ & 301 & 567 \\
\hline Refined & COV318 & $1 / 5(20.0)$ & ND & $5 / 11(45.5)$ & 63 & 148 \\
$(\mathrm{n}=11)$ & COV504 & $1 / 3(33.3)$ & ND & $3 / 11(27.3)$ & 27 & 66 \\
& OVCAR3 & $5 / 8(62.5)$ & ND & $7 / 11(63.6)$ & 95 & 169 \\
& SKOV3 & $7 / 8(87.5)$ & ND & $9 / 11(81.8)$ & 85 & 169 \\
\hline
\end{tabular}

ND: not determined; overall methylation represents the number of genes methylated of the total of 25 (classic) or 11 (refined) genes analyzed by MSP

Comparison of genes identified by candidate, BROAD and DEEP, and pharmacological approach

Comparing the three gene lists showed that CHFR, IRS1, PLLP, SFRP4, and TRIP13 were present in more than one gene list, whereas the majority of genes were unique. None of these genes was present in the gene lists of all three approaches. Of the 18 candidate genes, 0 genes were present in the BROAD and DEEP gene list; 2/19 (10.5\%) genes were present in the gene list derived from the pharmacological approach. The candidate genes CHFR and SFRP4 were also detected in the NT of SKOV3. CHFR methylation was not detected in SBTs or SCAs. Methylation of SFRP4 was more frequently detected in FTs as compared to SBTs and SCAs (Table 2). None of the 19 genes analyzed by BROAD and DEEP analysis was present in the candidate gene list. TRIP13 and PLLP $(2 / 19 ; 10.5 \%)$ were also present in the NT of COV504 and SKOV3 respectively. Although the MSP primers were designed within the sequence motif region, BROAD and DEEP analysis criteria were met, and the genes were present in a NT, primary tumors showed low methylation frequencies of PLLP (SBT 1/32 (3.1\%) and SCA 5/63 (7.9\%)) and TRIP13 (0/32 and 3/62 (4.8\%)) respectively. The gene list of the pharmacological approach contained 36 genes, of which 0 genes were in the candidate gene list and only IRS1 (1/36; 2.8\%) was also present in the BROAD and DEEP gene list. The MSP primers were designed around the TSS and methylation was detected in 3 of $6(50.0 \%)$ cell lines. IRS1 methylation was not assessed in SBTs and SCAs since 14/21 (66.7\%) FTs were methylated. 


\section{Discussion}

Identification of genes silenced by promoter $\mathrm{CpG}$ island hypermethylation might provide novel insights in the biology of ovarian cancer and might identify potential novel TSGs and biomarkers. Little information on serous ovarian cancer epigenetics is available. The majority of studies analyzed all epithelial ovarian cancer subtypes together. Since evidence is emerging that the subtypes represent distinct genetic and epigenetic entities, discrimination between the histological subtypes in studying cancer epigenetics is important [28-30].

The candidate gene approach revealed that genes that are frequently methylated in other cancer types are not as frequently methylated in serous ovarian cancer and vice versa, confirming other data that promoter $\mathrm{CpG}$ island hypermethylation profiles can differ per tumor type $[21,31]$. An interesting gene in this respect is GATA4, reported to be silenced and methylated in 70\% of colorectal carcinomas (CRC) [32]. In the present study, methylation was detected in only $4 / 30$ (13.3\%) SBTs and 4/62 (6.5\%) SCAs, despite previous observations that GATA4 is frequently silenced in ovarian cancer. Caslini et al. [33] showed that GATA4 silencing in ovarian cancer was associated with histone $\mathrm{H} 3$ and $\mathrm{H} 4$ hypoacetlyation and loss of H3K4 tri-methylation and that TSA treatment restored GATA4 expression in ovarian cancer cell lines. In our pharmacological approach, GATA4 was upregulated by DAC (1.73-2.01 fold) as well as by TSA (1.91-2.87 fold). These data show that different epigenetic mechanisms can regulate expression of the same gene in different cancer types. Another interesting finding was that serous ovarian cancer, at least for the genes analyzed in this study, differs from other cancer types regarding the frequency of promoter CpG island hypermethylation. A previous study showed relatively low frequencies of methylation in approximately 1,200 unselected CpG islands as determined by restriction landmark genomic scanning in breast, head and neck, and testicular tumors, whereas methylation was more pronounced in colon tumors, gliomas and acute myeloid leukemia [31]. Although many tumors share methylation for a given gene, cancer types have unique promoter hypermethylation profiles [21]. To identify novel methylation markers for serous ovarian cancer, it is important to search for methylation markers that are associated with decreased expression and one approach to directly study this is pharmacological unmasking of hypermethylated genes and subsequent validation of methylation in ovarian cancer cell lines. The number of genes in the TT and NT of CRC cell lines is higher (range 235-819 and 759-1749 respectively) than in the tiers of the ovarian cancer cell lines (123-303 and 391-831 respectively) (Table 4). The estimated size of the hypermethylome is also larger in CRC (532-1389) as compared to the ovarian hypermethylome, which was estimated at 123 to 817 genes, depending on cell line analyzed. This is another indication that serous ovarian cancers show less methylation when compared to e.g. CRC and a well-considered approach needs to be undertaken to identify candidate genes. 
Table 4: Hypermethylomes of ovarian and colorectal cancer cell lines

\begin{tabular}{lllllll}
\hline $\begin{array}{l}\text { Tumor } \\
\text { type }\end{array}$ & Cell line & $\begin{array}{l}\text { \# of } \\
\text { genes } \\
\text { (TT) }\end{array}$ & $\begin{array}{l}\text { \# of } \\
\text { genes } \\
\text { (NT) }\end{array}$ & $\begin{array}{l}\text { Calculated } \\
\text { hypermethylated } \\
\text { genes TT }\end{array}$ & $\begin{array}{l}\text { Calculated } \\
\text { hypermethylated } \\
\text { genes NT }\end{array}$ & $\begin{array}{l}\text { Estimated size } \\
\text { hypermethylome }\end{array}$ \\
\hline Ovarian & COV318 & 303 & 831 & 101 & 276 & 377 \\
& COV504 & 123 & 391 & 123 & 0 & 123 \\
& OVCAR3 & 286 & 781 & 57 & 223 & 280 \\
& SKOV3 & 301 & 567 & 250 & 567 & 817 \\
\hline CRC & CACO2 & 235 & 774 & 153 & 379 & 532 \\
& COLO320 & 693 & 1337 & 450 & 655 & 1105 \\
& HCT116 & 532 & 1190 & 484 & 582 & 1067 \\
& HT29 & 618 & 1260 & 402 & 617 & 1019 \\
& RKO & 819 & 1749 & 532 & 857 & 1389 \\
& SW480 & 318 & 759 & 207 & 372 & 579 \\
\hline
\end{tabular}

Calculated hypermethylated genes of each tier shows the number of genes methylated times the number of genes present in the corresponding tier (e.g. 33.3\% of 303); the number of calculated hypermethylated genes TT plus NT is presented in the column estimated size of hypermethylome. Data CRC (colorectal cancer) adapted from [19]

Overall methylation frequencies in ovarian cancer cell lines appeared higher in genes identified by the refined approach as well as in the cell lines OVCAR3 (classic approach 9/25 (36.0\%) and refined 7/11 (63.6\%)) and SKOV3 (19/25 (76.0\%) and 9/11 (81.8\%) respectively) as compared to COV318 (6/25 (24.0\%) and 5/11 (45.5\%)) and COV504 (6/25 (24.0\%) and 3/11 (27.3\%)). CpG island methylator phenotype (CIMP) [34], defined as high methylation frequencies of methylation in a single sample, is a phenomenon extensively described in CRC [34]. Ten loci were investigated for the methylation status in ovarian carcinomas. Significant hypermethylation was detected in a proportion of these tumors, irrespective of histological subtype, indicating that a CIMP phenotype might also exist in ovarian tumors [35]. When comparing the three different approaches used for the identification of novel hypermethylated genes in serous ovarian cancer, five genes were shared between two gene lists: CHFR and SFRP4 were present in candidate gene and pharmacological unmasking lists and IRS1, PLLP and TRIP13 were present in both BROAD and DEEP and pharmacological gene lists. Thus, the majority of genes were uniquely identified by one approach. Because methylation of the candidate genes was not very frequently observed, it is not surprising that these genes were not identified in the TTs of the cell lines. It is however unexpected that not more genes identified by BROAD and DEEP analyses appear in the list generated by the pharmacological approach and vice versa. It was predicted that genes that are more prone to hypermethylation due to 1 ) specific sequence motifs in the promoter; or 2) respond to DAC, show up in both gene lists because these genes were chosen on purpose. Selection of genes by pharmacological unmasking occurred if DAC treatment induced expression at least 1,41 fold. Genes with lower re-expression levels were ignored, whereas these genes might be present in the BROAD and DEEP gene lists and might as well be methylated. It has also been described that genes can be expressed upon DAC treatment while being unmethylated [36, 37]. In this case, it is expected that the gene of interest is not present in the BROAD and DEEP gene list. Although the DEEP analysis was successful in previous studies $[18,38]$, it is possible that the methylation prone sequence motifs differ between cancer types and other motifs are methylated in ovarian cancer. Two studies identified seven (TCCCCC*C, TTTCCT*C, TCC*CC*CCC, GGAG*AAG, GAGA*AAG, GCCACCCC, and GAGGAGG**G) and 
five (GGCTGCGGGGGCAGCAGCTG, AAGAAGGGAGAGAAGGAGGAA, TCCTCTCCCTTGTCTTCCTCCTCCTCCTC, GGGGTGGGGGAGGGGGAGGAG, CTCTCCCAAGC) methylation prone sequence motifs respectively $[39,40]$. These sequences are not shared with the sequence motifs used in the current study. Another explanation for the low frequency of methylation of the examined genes and the low specificity might be related to the MSP assay that was used to analyze methylation. This method focuses on a restricted region of the $\mathrm{CpG}$ island and only a limited number of $\mathrm{CpG}$ dinucleotides are examined, therefore only a fraction of the information of a given $\mathrm{CpG}$ island is provided. Evidence is emerging that the frequency and type (e.g. tissue-specific or cancer-specific) of promoter $C p G$ island hypermethylation can vary significantly between different positions in the same CpG island. In addition, it has been shown recently that the location of the MSP primers is crucial for the detection of clinical associations. Promoter hypermethylation of GREM1 was associated with poor prognosis in renal cell carcinomas when a region downstream of the TSS (region iii +311 to +472 relative to TSS) was methylated, whereas two examined regions upstream of the TSS (region $i$ 648 to -462 and region ii -236 to -140 ) did not show this clinical association [41]. The low methylation frequencies might therefore be associated with the examined promoter region. A recent review showed that BRCA1 methylation frequencies in ovarian cancer differed according to techniques and was dependent on the location of the MSP primers [42]. Thus, it is very well possible that if other regions of the promoter $\mathrm{CpG}$ island of the gene of interest are examined, methylation frequencies will increase or methylation becomes functionally significant (e.g. cancer-specific methylation). Novel, (epi)genome-wide sequencing technology such as MBD affinity genome sequencing [43] enables rapid generation of accurate and detailed information of the entire epigenome [44]. This will lead to novel insights in methylation patterns that probably explain part of the discrepancies observed between the approaches used in this study and will identify sensitive and specific methylation markers. Although next generation sequencing is currently expensive, this technology will become affordable soon enabling sequencing of individual samples and therapy can be individualized based on the epigenetic information gathered by sequencing. This study aimed to identify methylation markers able to discriminate between SBTs and SCAs and have the potential to serve as diagnostic or therapeutic markers. Methylated genes were considered as candidate methylation marker if methylation was detected in at least $35 \%$ of SBTs or SCAs. Eight genes (ACTN1, BHMT2, BRCA1, CDO1, IL6R, RASSF1A, TNFRSF1OB, and WIF1) met this criterion. The majority $(51 / 60 ; 85.0 \%)$ of genes was methylated in less than $35 \%$, of which $37 / 51(72.5 \%)$ showed very low $(<15 \%)$ methylation frequencies. Although these markers are not suitable for discriminating between SBTs and SCAs, these genes are potentially interesting for other purposes, such as prediction of prognosis or response to therapy. False positive candidate genes (either upregulation after DAC treatment although the gene did not appear to be methylated or promoter CpG island hypermethylation detected in ovarian cancer cell lines, whereas no or low methylation in SBTs and SCAs was detected) were frequently identified by using the "classic" algorithm of data analysis. These phenomena have been described before $[28,36,45]$. Therefore, the microarray results were re-analyzed using more stringent criteria ("refined approach") to reduce this number. By using the latter approach, BHMT2 and CDO1, two novel 
methylation markers, were identified. BHMT2 and CDO1 were frequently methylated in serous ovarian cancer and were differentially methylated between SBTs and SCAs. CDO1 methylation was detected in 2/22 (9.1\%) FTs, in 3/30 (10.0\%) SBTs and in 42/64 $(65.6 \%)$ SCAs $(p<0.001)$, indicating that this gene might be used as a diagnostic marker. However, larger study populations are required to validate these results and analysis of panels of hypermethylated markers to increase the sensitivity of methylation markers guiding the diagnosis might be useful.

Since promoter CpG island hypermethylation of BHMT2 has yet not been described, this gene was chosen for more extensive analysis for tumor suppressive properties. The results of that study are presented in the next chapter of this thesis.

\section{Acknowledgements}

Sandra van den Bosch, Sarah Hulsmans, and Kim Wouters are gratefully acknowledged for technical support. 


\section{References}

1. Cannistra SA. Cancer of the ovary. N Engl J Med 2004;351(24):2519-29.

2. Prat J. Surface epithelial-stromal tumors: Serous tumors: Saunders; 2004: 83-111

3. Ferlay J, Shin HR, Bray F, Forman D, Mathers C, Parkin DM. Estimates of worldwide burden of cancer in 2008: GLOBOCAN 2008. Int J Cancer 2010;127(12):2918-27.

4. Crispens MA. Borderline ovarian tumours: a review of the recent literature. Curr Opin Obstet Gynecol 2003;15(1):39-43.

5. Leitao MM, Jr., Boyd J, Hummer A, et al. Clinicopathologic analysis of early-stage sporadic ovarian carcinoma. Am J Surg Pathol 2004;28(2):147-59.

6. Young RC, Walton LA, Ellenberg SS, et al. Adjuvant therapy in stage I and stage II epithelial ovarian cancer. Results of two prospective randomized trials. N Engl J Med 1990;322(15):1021-7.

7. Sieben NL, Macropoulos P, Roemen GM, et al. In ovarian neoplasms, BRAF, but not KRAS, mutations are restricted to low-grade serous tumours. J Pathol 2004;202(3):336-40.

8. Singer $\mathrm{G}$, Oldt $\mathrm{R}, 3 \mathrm{rd}$, Cohen $\mathrm{Y}$, et al. Mutations in BRAF and KRAS characterize the development of low-grade ovarian serous carcinoma. J Natl Cancer Inst 2003;95(6):484-6.

9. Shih le M, Kurman RJ. Molecular pathogenesis of ovarian borderline tumors: new insights and old challenges. Clin Cancer Res 2005;11(20):7273-9.

10. Salani R, Kurman RJ, Giuntoli R, 2nd, et al. Assessment of TP53 mutation using purified tissue samples of ovarian serous carcinomas reveals a higher mutation rate than previously reported and does not correlate with drug resistance. Int J Gynecol Cancer 2008;18(3):48791.

11. Herman JG, Baylin SB. Gene silencing in cancer in association with promoter hypermethylation. N Engl J Med 2003;349(21):2042-54.

12. Sellar GC, Watt KP, Rabiasz GJ, et al. OPCML at $11 \mathrm{q} 25$ is epigenetically inactivated and has tumor-suppressor function in epithelial ovarian cancer. Nature genetics 2003;34(3):337-43.

13. Teodoridis JM, Hall J, Marsh S, et al. CpG island methylation of DNA damage response genes in advanced ovarian cancer. Cancer Res 2005;65(19):8961-7.

14. Esteller M, Silva JM, Dominguez G, et al. Promoter hypermethylation and BRCA1 inactivation in sporadic breast and ovarian tumors. J Natl Cancer Inst 2000;92(7):564-9.

15. Wiley $A$, Katsaros $D$, Chen $H$, et al. Aberrant promoter methylation of multiple genes in malignant ovarian tumors and in ovarian tumors with low malignant potential. Cancer 2006;107(2):299-308.

16. Fang F, Balch C, Schilder J, et al. A phase 1 and pharmacodynamic study of decitabine in combination with carboplatin in patients with recurrent, platinum-resistant, epithelial ovarian cancer. Cancer 2010;116(17):4043-53.

17. www.clinicaltrials.gov

18. Hoque MO, Kim MS, Ostrow KL, et al. Genome-wide promoter analysis uncovers portions of the cancer methylome. Cancer research 2008;68(8):2661-70.

19. Schuebel KE, Chen W, Cope L, et al. Comparing the DNA hypermethylome with gene mutations in human colorectal cancer. PLoS Genet 2007;3(9):1709-23.

20. Smyth GK. Limma: linear models for microarray data. Bioinformatics and Computational Biology Solutions using R and Bioconductor: Springer, New York; 2005.

21. Esteller M, Corn PG, Baylin SB, Herman JG. A gene hypermethylation profile of human cancer. Cancer Res 2001;61(8):3225-9.

22. Sieben NL, Oosting J, Flanagan AM, et al. Differential gene expression in ovarian tumors reveals Dusp 4 and Serpina 5 as key regulators for benign behavior of serous borderline tumors. J Clin Oncol 2005;23(29):7257-64.

23. McGarvey KM, Van Neste L, Cope L, et al. Defining a chromatin pattern that characterizes DNA-hypermethylated genes in colon cancer cells. Cancer research 2008;68(14):5753-9.

24. Derks S, Lentjes $M H$, Hellebrekers DM, de Bruine AP, Herman JG, van Engeland M. Methylation-specific PCR unraveled. Cell Oncol 2004;26(5-6):291-9.

25. Herman JG, Graff JR, Myohanen S, Nelkin BD, Baylin SB. Methylation-specific PCR: a novel PCR assay for methylation status of CpG islands. Proceedings of the National Academy of Sciences of the United States of America 1996;93(18):9821-6. 
26. van Engeland M, Weijenberg MP, Roemen GM, et al. Effects of dietary folate and alcohol intake on promoter methylation in sporadic colorectal cancer: the Netherlands cohort study on diet and cancer. Cancer research 2003;63(12):3133-7.

27. De Meyer T, Bijsmans I, Van de Vijver K, et al. E2Fs mediate a fundamental cell-cycle deregulation in high-grade serous ovarian carcinomas. J Pathol 2008;217 (1):14-20.

28. Houshdaran S, Hawley S, Palmer C, et al. DNA methylation profiles of ovarian epithelial carcinoma tumors and cell lines. PloS one 2010;5(2):e9359.

29. Ho CM, Lin MC, Huang SH, et al. PTEN promoter methylation and LOH of 10q22-23 locus in PTEN expression of ovarian clear cell adenocarcinomas. Gynecologic oncology 2009;112(2):307-13.

30. Torng PL, Lin CW, Chan MW, Yang HW, Huang SC, Lin CT. Promoter methylation of IGFBP-3 and p53 expression in ovarian endometrioid carcinoma. Molecular cancer 2009;8:120.

31. Costello JF, Fruhwald MC, Smiraglia DJ, et al. Aberrant CpG-island methylation has nonrandom and tumour-type-specific patterns. Nature genetics 2000;24(2):132-8.

32. Hellebrekers DM, Lentjes MH, van den Bosch SM, et al. GATA4 and GATA5 are potential tumor suppressors and biomarkers in colorectal cancer. Clin Cancer Res 2009;15(12):39907.

33. Caslini C, Capo-chichi CD, Roland IH, Nicolas E, Yeung AT, Xu XX. Histone modifications silence the GATA transcription factor genes in ovarian cancer. Oncogene 2006;25(39):544661.

34. Issa JP. CpG island methylator phenotype in cancer. Nat Rev Cancer 2004;4(12):988-93.

35. Strathdee $\mathrm{G}$, Appleton $\mathrm{K}$, Illand $\mathrm{M}$, et al. Primary ovarian carcinomas display multiple methylator phenotypes involving known tumor suppressor genes. Am J Pathol 2001;158(3):1121-7.

36. Hellebrekers DM, Melotte V, Vire E, et al. Identification of epigenetically silenced genes in tumor endothelial cells. Cancer research 2007;67(9):4138-48.

37. Nguyen CT, Weisenberger DJ, Velicescu M, et al. Histone H3-lysine 9 methylation is associated with aberrant gene silencing in cancer cells and is rapidly reversed by 5 -aza-2'deoxycytidine. Cancer research 2002;62(22):6456-61.

38. Ongenaert $\mathrm{M}$, Wisman $\mathrm{GB}$, Volders $\mathrm{HH}$, et al. Discovery of DNA methylation markers in cervical cancer using relaxation ranking. BMC medical genomics 2008;1:57.

39. Feltus FA, Lee EK, Costello JF, Plass C, Vertino PM. Predicting aberrant CpG island methylation. Proceedings of the National Academy of Sciences of the United States of America 2003;100(21):12253-8.

40. Feltus FA, Lee EK, Costello JF, Plass C, Vertino PM. DNA motifs associated with aberrant CpG island methylation. Genomics 2006;87(5):572-9.

41. van Vlodrop IJ, Baldewijns MM, Smits KM, et al. Prognostic Significance of Gremlin1 (GREM1) Promoter CpG Island Hypermethylation in Clear Cell Renal Cell Carcinoma. Am J Pathol 2010;176 (2):575-84.

42. Senturk E, Cohen S, Dottino PR, Martignetti JA. A critical re-appraisal of BRCA1 methylation studies in ovarian cancer. Gynecologic oncology 2010;119(2):376-83.

43. Serre $D$, Lee $B H$, Ting AH. MBD-isolated Genome Sequencing provides a high-throughput and comprehensive survey of DNA methylation in the human genome. Nucleic acids research 2010;38(2):391-9.

44. Laird PW. Principles and challenges of genome-wide DNA methylation analysis. Nature reviews 2010;11(3):191-203.

45. Smiraglia DJ, Rush LJ, Fruhwald MC, et al. Excessive CpG island hypermethylation in cancer cell lines versus primary human malignancies. Human molecular genetics 2001;10(13):1413-9. 


\section{CHAPTER 6}

Betaine-homocysteine methyltransferase 2 (BHMT2) is a candidate tumor suppressor gene frequently inactivated by promoter CpG island hypermethylation in serous ovarian cancer

Ingrid T.G.W. Bijsmans, Leander Van Neste, Wim Van Criekinge, Edith van den Boezem, Kathleen L. Daenen, Koen K. Van de Vijver, Adriaan P. de Bruïne, Nathalie L.G. Sieben and Manon van Engeland

In preparation 


\begin{abstract}
Pharmacological unmasking of promoter CpG island hypermethylated genes in ovarian cancer cell lines identified Betaine-homocysteine methyltransferase 2 (BHMT2) as a gene frequently methylated in serous ovarian cancer. BHMT2 encodes a methyltransferase catalyzing the remethylation of homocysteine to methionine. Three regions spanning the first CpG island of $B H M T 2$ (region M1: -192 to -37 relative to the transcription start site (TSS), M2: 50 to +10 , and M3: +43 to +152 ) were analyzed for methylation by methylation specific PCR (MSP) in 6 ovarian cancer cell lines, 21 fallopian tubes (FTs), 32 serous borderline tumors (SBTs) and 64 carcinomas (SCAs). All ovarian cancer cell lines showed dense methylation. Treatment of five of the cell lines (COV318, COV504, OVCAR3, OV90 and SKOV3) with the demethylating agent 5-aza-2'deoxycytidine restored BHMT2 mRNA expression, ranging from 2 to 950-fold induction. In addition, we show cancer-specific methylation at the region downstream of the TSS (+43 to +152$): 1 / 19(5.3 \%)$ of FTs, $10 / 28$ (35.7\%) of SBTs and $47 / 64(73.4 \%)$ of SCAs showed BHMT2 promoter CpG island hypermethylation (SBT versus SCA: $\mathrm{p}=0.001$ ). Bisulfite sequencing performed on 2 methylated and 2 unmethylated SCAs confirmed MSP results. BHMT2 mRNA levels were lower in SBTs and SCAs as compared to FTs ( $p=0.015$ and $p=0.002$ respectively). The potential tumor suppressive properties of $B H M T 2$ were studied in an in vitro model. To this end, the ovarian cancer cell line SKOV3 was transfected with BHMT2. Overexpression of BHMT2 reduced colony formation, invasion and migration, indicating that BHMT2 is a novel candidate tumor suppressor gene in serous ovarian cancer.
\end{abstract}




\section{Introduction}

Epithelial ovarian cancer is a leading cause of death in women suffering from cancer of the female genital tract. Since obvious symptoms are absent in early stages, this disease is usually diagnosed at an advanced stage [1]. Worldwide, ovarian cancer was estimated to account for 225,000 cases and 140,000 deaths in 2008 [2].

Serous carcinomas (SCAs) are the most common ovarian tumors. It has been thought that these tumors arise from the ovarian surface epithelium [3]. However, current evidence suggests that high-grade SCAs (HG-SCAs) might develop from the distal fallopian tube fimbriae [4-6] or endometrium [7]. Serous borderline tumors (SBTs) belong to an intermediate group of ovarian tumors and are characterized by stratified epithelial cells, varying nuclear atypia, and lack of destructive stromal invasion $[3,8]$. SBT patients may present with tumor deposits, so-called implants. Nevertheless, the 5year survival remains excellent (90-95\%). The most important discriminator between SBTs and SCAs is the presence of stromal invasion in SCAs [3]. Long-term survival rates among patients with early-stage (FIGO stage I/II) SCA range from 80 to $90 \%$, whereas the 5-year survival rate of advanced-stage (III/IV) SCA is only $25 \%$. Due to non-specific symptoms in early stages, in most cases the tumor has spread to the upper abdomen at time of diagnosis, resulting in HG-SCA and a poor prognosis [3]. Many serous tumors show genetic alterations in BRAF, KRAS or TP53. BRAF and KRAS mutations occur exclusively in SBTs, together accounting for approximately $60 \%$ of SBTs $[9,10]$. The tumor suppressor gene TP53 is mutated in up to $80 \%$ of HG-SCAs, while the prevalence is very low (0-8\%) in SBTs and LG-SCAs [3, 11, 12]. To increase the knowledge about the epigenetic profile of serous ovarian tumors, promoter CpG island hypermethylation was analyzed by pharmacological unmasking of hypermethylated TSGs in ovarian cancer cell lines, as described in detail in Chapter 5 of this thesis. One of the genes found to be frequently hypermethylated in serous ovarian cancer cell lines and primary tumors is BHMT2. The current study was conducted to determine the extent and biological relevance of $B H M T 2$ promoter $C p G$ island hypermethylation in SBTs and SCAs. We analyzed BHMT2 promoter CpG island hypermethylation in histological normal fallopian tubes (FTs), SBTs, and SCA. The in vitro tumor suppression function of BHMT2 was determined by colony formation, invasion and migration assays. 


\section{Materials and methods}

\section{Cell lines and patient material}

Ovarian cancer cell lines SKOV3 and OVCAR3 were purchased from ATCC. COV318, COV413A, and COV504 were kindly provided by Dr. J. van Eendenburg (Leiden University Medical Center (LUMC), Leiden, The Netherlands). All cell lines were maintained in RPMI-1640 medium (Gibco, Invitrogen) supplemented with $10 \%$ fetal calf serum (FCS; Gibco) and 1\% glutamax (Gibco). Cell line OV90 (ATCC) was cultured in complete growth medium (1:1 mixture of medium 199 and MCDB105 (Sigma-Aldrich, St. Louis, USA)), supplemented with $10 \%$ FCS and $1 \%$ glutamax. Fresh-frozen or formalin-fixed paraffin-embedded tissue of histological normal fallopian tubes (FTs; $\mathrm{n}=23$ ), $26 \mathrm{FTs}$ derived from diseased ovaries (including benign, borderline and cancer), 32 SBTs and 64 SCAs were obtained from the Maastricht Pathology Tissue Collection (MPTC; MUMC), the archive of the Department of Pathology of LUMC, and Stichting PAMM (Eindhoven, The Netherlands; kindly provided by Thom Boerman). Diagnoses were confirmed by experienced gynecologic pathologists (KVdV, NS) by reviewing H\&E slides. See Table 1 for detailed clinicopathological characteristics. Baseline characteristics (International Federation of Gynecology and Obstetrics (FIGO) stage, age at diagnosis, cancer related death and overall mortality) of the samples derived from the diverse hospitals did not differ, therefore, these were considered as one group (data not shown). None of the patients received chemotherapy prior to surgery. Collection, storage and use of tissue and patient data were performed in agreement with the "Code for Proper Secondary Use of Human Tissue in The Netherlands" (http://www.fmwv.nl). This study was approved by the Medical Ethical Committee of the MUMC.

Table 1: Clinicopathological characteristics

\begin{tabular}{lll}
\hline Characteristics & & Patients (n=145) \\
\hline & FT histologically normal & $\mathrm{n}(\%)$ \\
& FT diseased & $26(15.9)$ \\
& SBT & $32(22.1)$ \\
& SCA & $64(44.1)$ \\
Age at diagnosis* & & \\
Years (range) & FT histologically normal & $31.3(20-40)$ \\
& FT diseased & $58.8(26-88)$ \\
& SBT & $46.1(19-77)$ \\
FIGO stage* & SCA & $60.7(21-87)$ \\
SBT & & \\
& & \\
SCA & stage I & $19(59.4)$ \\
& stage II & $1(3.1)$ \\
& stage III & $11(34.4)$ \\
& stage I & $10(15.6)$ \\
& stage II & $5(7.8)$ \\
& stage III & $45(70.3)$ \\
& stage IV & $3(4.7)$ \\
\hline
\end{tabular}

${ }^{*}$ Age and FIGO stage were unavailable for 2 patients (1 SBT, 1 SCA) 
DAC and TSA treatment of ovarian cancer cell lines

To inhibit DNA methylation, ovarian cancer cells at $10 \%$ confluency were treated with $5 \mu \mathrm{M}$ 5-aza-2'-deoxycytidine (DAC (Sigma-Aldrich); $1 \mathrm{mM}$ stock solution in PBS) for 96 hours, replacing DAC-containing medium every 24 hours. Treatment with $300 \mathrm{nM}$ Trichostatin A (TSA (Sigma-Aldrich); $1.5 \mathrm{mM}$ stock solution in ethanol) was performed on $30 \%$ confluent cells for 18 hours to prevent histone deacetylation. In parallel, control cells (mock) were treated with equal volumes of PBS or ethanol without drugs respectively.

\section{DNA and RNA isolation}

Tissue (histologically normal FT, SBT or SCA) from ten haematoxylin-stained $10 \mu \mathrm{m}$ sections was microdissected with a $10 \mathrm{G}$ needle under transmitted light microscopy. The tumor sections contained at least $75 \%$ tumor cells. FTs derived from diseased ovaries were macrodissected. DNA was extracted using Puregene DNA isolation kit (Gentra Systems, BIOzymTC; Landgraaf, The Netherlands) according to manufacturer's description. RNA was isolated using Sigma GenElute Mammalian Total RNA Kit (SigmaAldrich) according to manufacturer's protocol.

\section{Pharmacologic microarray analysis and candidate gene selection}

Microarray analysis was performed on DAC, TSA and mock treated cell lines COV318, COV504, OVCAR3 and SKOV3, as described in detail in Chapter 5 of this thesis. In brief, RNA was isolated, labeled and hybridized on whole genome microarrays, followed by candidate TSG identification. Selection criteria were: 1) basal expression was low/absent in wild-type ovarian cancer cell lines; 2) re-expression was detected after DAC treatment, while 3) TSA response was absent. The DAC-responsive zone contains two tier regions: top tier and next tier. The top tier (TT) is defined as a zone in which gene expression increased at least 2-fold after DAC treatment, while unaffected by TSA treatment. The next tier (NT) contains candidate genes of which DAC treatment increased gene expression between 1.41 and 2-fold and TSA treatment did not increase or decrease gene expression. Using this approach, BHMT2 was identified as a candidate methylation marker.

Sodium bisulfite treatment, methylation specific $P C R(M S P)$, and bisulfite sequencing Sodium bisulfite treatment was performed on $500 \mathrm{ng}$ genomic DNA using the EZ DNA methylation $\mathrm{Kit}^{\mathrm{TM}}$ (Zymo research, Orange, CA, USA) according to manufacturer's description. Determination of the transcription start site (TSS) was performed using DBTSS (http://dbtss.hgc.jp). CpGPlot (http://www.ebi.ac.uk/Tools/emboss/ cpgplot/index.html) was used for CpG island confirmation using default settings (length CpG island $>200$ base pairs, GC content $>50 \%$ ). Three MSP primer pairs (M1: 192 to -37 relative to TSS, $M 2:-50$ to +10 and $M 3:+43$ to +152 ) covering the first CpG island of BHMT2 were designed (Figure 2A and Supplementary Table S1). MSP analysis was performed by a nested approach in 96-well PCR plates (Greiner Bio-One, Alphen a/d Rijn, The Netherlands), as reported previously [13-15]. Validation of promoter CpG island hypermethylation of 2 unmethylated and 2 methylated SCAs was performed by bisulfite sequencing. Two overlapping regions (B1: $158 \mathrm{bp}, 19 \mathrm{CpGs},-192$ to -35 relative to TSS; and B2: $218 \mathrm{bp}, 24 \mathrm{CpGs},-63$ to +154 ; Figure $2 \mathrm{~A}$ ) were analyzed. PCR 
products were cloned into pCR2.1 (TOPO-TA cloning kit; Invitrogen, Breda, The Netherlands). Single bacterial clones were sequenced using BigDye ${ }^{\circledR}$ Terminator v3.1 Cycle Sequencing Kit (Applied Biosystems). For each sample, six clones, if available, were subjected to sequencing. Primer sequences and PCR conditions are provided in Supplementary Table S1.

\section{Quantitative RT-PCR}

Quantitative RT-PCR was performed as described previously [16]. Briefly, one microgram of RNA was reverse-transcribed using the iScript ${ }^{\mathrm{TM}}$ cDNA Synthesis kit (Biorad, Veenendaal, The Netherlands). $\mathrm{IQ}^{\mathrm{TM}} \mathrm{SYBR}^{\circledR}$ Green Supermix (Biorad) was used for quantitative RT-PCR. The reference genes cyclophilin $A$ or $\beta$-actin were used for normalization. Cyclophilin $A$ was used for primary tumors and cell transfection experiments; $\beta$-actin for mRNA expression of DAC, TSA or mock treated cell lines. Primer sequences are listed in Supplementary Table S1.

\section{Protein isolation and western blotting}

Cytoplasmic extracts were prepared using NE-PER nuclear and cytoplasmic extraction reagents (Pierce Biotechnology, IL, USA) containing protease inhibitors (complete protease inhibitor cocktail tablets; Roche Applied Science, Mannheim, Germany) following manufacturer's protocol. Proteins were separated by $10 \%$ SDS-PAGE and transferred to a nitrocellulose membrane (Whatman), followed by blocking in $4 \%$ nonfat dry milk in $0.1 \%$ Tween-20/PBS. Membranes were probed with rabbit polyclonal anti-HA (Y-11; 0.2 Mg/ml; Santa Cruz Biotechnology Inc., Santa Cruz, CA, USA), followed by incubation with the secondary polyclonal goat anti-rabbit immunoglobulins/HRP antibody $(1.5 \mu \mathrm{g} / \mathrm{ml}$; DAKO). Bound antibody was detected using Pierce ECL Western Blotting Substrate (Thermo Scientific, Rockford, IL, USA). Actin (clone C4; MP Biomedicals) was included as loading control.

\section{Transient BHMT2 transfection}

HA-tagged full-length BHMT2 (pcDNA3.1-BHMT2-HA), a kind gift by Professor R. Weinshilboum (Department of Molecular Pharmacology and Experimental Therapeutics, Mayo Clinic, Minnesota, USA), was subcloned into pcDNA3 (Invitrogen). SKOV3 cells were transfected with pcDNA3-BHMT2-HA or empty vector (pcDNA3) using FuGENE ${ }^{\circledR} 6$ Transfection reagent (Roche Diagnostics $\mathrm{GmbH}$, Mannheim, Germany) according to manufacturer's protocol.

\section{Colony formation assay}

SKOV3 cells were transfected with pcDNA3-BHMT2-HA or empty vector and harvested 48 hours post-transfection. Cells were seeded $1: 2$ in six-well plates. The next day, G418 (600 $\mu \mathrm{g} / \mathrm{ml}$; GibcoBRL, Life Technologies, Paisley, Scotland) was added to the medium to select for plasmid-expressing cells. After 14 days of selection, colonies were stained using Giemsa's azur eosine methylene blue (Merck, Darmstadt, Germany). Colonies from three independent experiments were counted using Image 1.42 software (National Institutes of Health, USA). 
In vitro invasion and migration assay

After transfection, cells were selected with $\mathrm{G} 418$ for 11 days. Assays were performed using $8 \mu \mathrm{m}$ pore size transwells (BD Biosciences, San Jose, CA, USA) coated with Matrigel (invasion) or uncoated (migration). SKOV3 cells were seeded at a concentration of $1 \times 10^{4}$ to $7 \times 10^{4}$ in RPMI-1640 supplemented with $1 \%$ FCS in the upper chamber. The lower chamber contained 10\% FCS-RPMI-1640 medium. After 24 hours, cells that remained on the upper surface of the chamber were removed by a cotton swab. Cells on the lower surface of the membrane were fixed in methanol, followed by $H \& E$ staining. Cells in two random microscopic fields per membrane from three independent experiments were counted.

\section{Data analysis}

Associations between patient characteristics of the different hospitals, the comparison of methylation frequencies between SBTs and SCAs, analyses of quantitative RT-PCR, colony formation, invasion and migration data were calculated using Pearson ChiSquare test or independent-Samples T Test (SPSS 15.0 for Windows) where appropriate. Results of colony formation, invasion and migration are given as mean values \pm standard error. All $p$-values are two-sided and $p \leq 0.05$ was considered statistically significant.

\section{Results}

BHMT2 is silenced by promoter CPG island hypermethylation and can be re-expressed by DAC in ovarian cancer cell lines

BHMT2 was present in the TT of the ovarian cancer cell lines COV504, OVCAR3 and SKOV3, as indicated by the orange spot in the scatter plots (Figure 1). The BHMT2 gene (NM_017614) is located at chromosome 5q13, contains 8 exons, spans 1700 bases, and encodes a protein of 363 amino acids. The BHMT2 promoter contains two CpG islands (defined as a region of $>200$ base pairs and a CG content of $>50 \%$ ) of 462 bp and 239 bp respectively. Island 1 is located from -288 to +172 relative to the transcription start site (TSS), island 2 from +304 to +542 . Evidence is emerging that the location of methylation can vary significantly within the promoter of a single gene. Therefore, three MSP primer pairs (M1: -192 to -37 relative to the TSS, M2: 50 to +10 , and M3: +43 to +152 ; Figure $2 \mathrm{~A}$ ) were designed in the first $\mathrm{CpG}$ island. BHMT2 promoter CpG island hypermethylation at the three regions was examined by MSP in the cell lines COV318, COV413A, COV504, OVCAR3, SKOV3, and OV90. All cell lines showed BHMT2 methylation at the three analyzed regions (data not shown). Since the second BHMT2 CpG island is not dense enough, it was impossible to design MSP primers for this region. To investigate whether methylation was associated with BHMT2 gene silencing, the cell lines COV318, COV504, OVCAR3, SKOV3, and OV90 were treated with the demethylating agent DAC. Basal BHMT2 mRNA expression was very low in OVCAR3, SKOV3 and OV90 cells, COV318 and COV504 showed higher basal BHMT2 mRNA levels (data not shown). DAC treatment restored mRNA expression approximately 2-fold in COV318 and OV90 (relative to mock treated cells), roughly 8-fold in COV504, and approximately 570 and 950-fold in OVCAR3 and SKOV3 respectively (Figure 2B). For 
inhibition of histone deacetylase activity, cells were exposed to Trichostatin A (TSA). Relative to mock cells, TSA restored expression in OV90 and OVCAR3 (1.2 and 57-fold respectively), whereas TSA was non-responsive in COV504 (0.4 fold) and SKOV3 (0.9 fold) (Figure 2B).
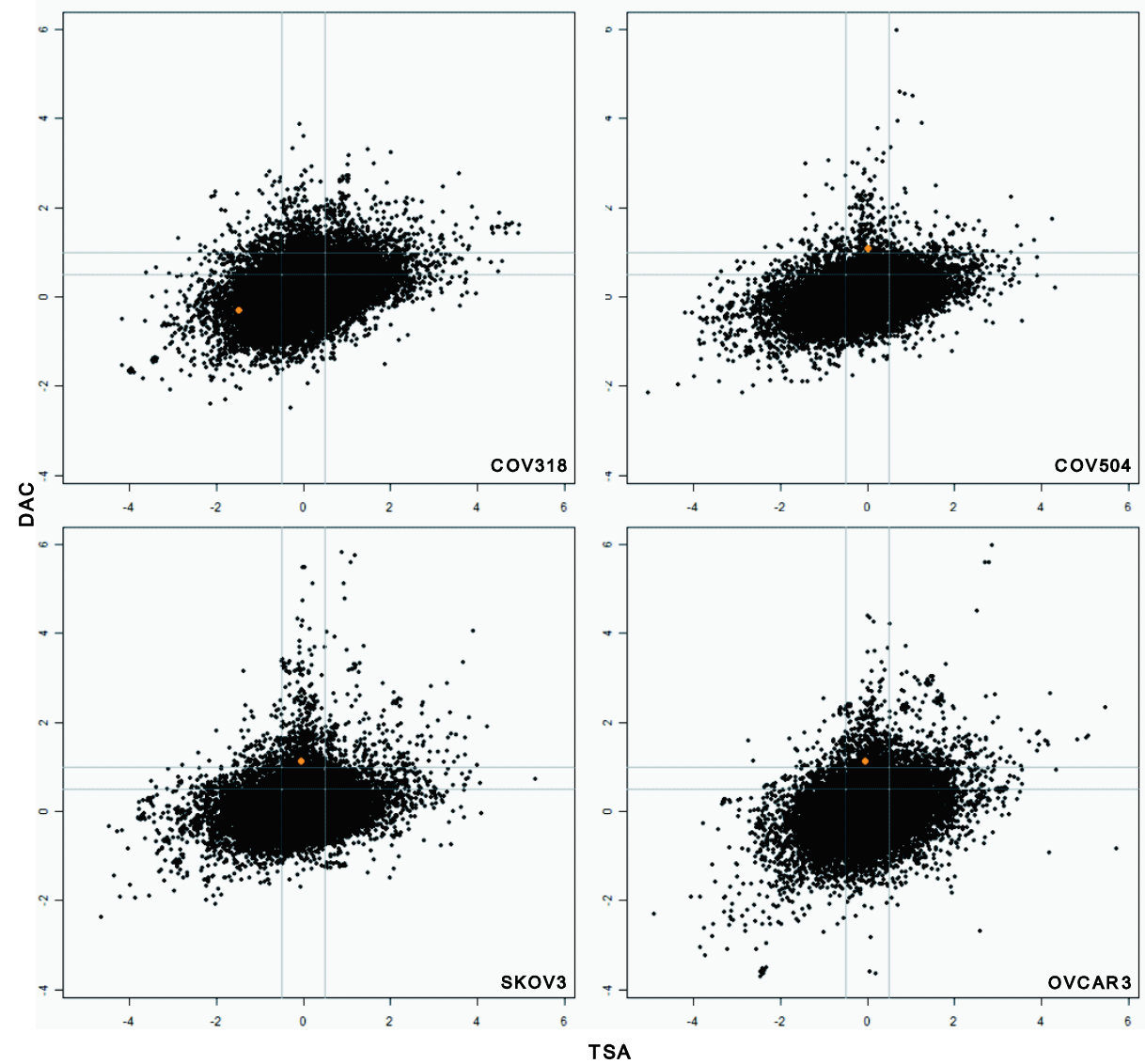

Figure 1: Illustration of gene-expression changes in DAC and TSA treated ovarian cancer cell lines Scatter-plots of the cell lines COV318, COV504, OVCAR3 and SKOV3 respectively. Individual genes are shown in black, BHMT2 is shown in orange. 
Epigenetic silencing of BHMT2 in serous ovarian cancer

Insert figure 2 
BHMT2 is frequently methylated in SBTs and SCAs

Next, we analyzed BHMT2 methylation in 23 histological normal FTs, 26 FTs derived from women with ovarian disease, 32 SBTs and 64 SCAs. BHMT2 promoter CpG island hypermethylation of regions M1 to M3 was detected in all FTs from diseased ovarian cases. However, histological normal showed a decrease in methylation frequency more downstream of the TSS: $12 / 19$ (63.2\%), 5/21 (23.8\%) and 1/19 (5.3\%) at M1, M2 and M3 respectively $(\mathrm{p}<0.001)$, whereas BHMT2 methylation in SBTs and SCAs was not significantly different (Table 2 ).

Table 2: BHMT2 promoter $\mathrm{CpG}$ island hypermethylation in serous tumors

\begin{tabular}{lllll}
\hline region & FT (\%) & SBT (\%) & SCA (\%) & p value* $^{*}$ \\
\hline M1 & $12 / 19(63.2)$ & $12 / 29(41.4)$ & $45 / 64(70.3)$ & 0.008 \\
M2 & $5 / 21(23.8)$ & $16 / 32(50.0)$ & $48 / 64(75.0)$ & 0.014 \\
M3 & $1 / 19(5.3)$ & $10 / 28(35.7)$ & $47 / 64(73.4)$ & 0.001 \\
\hline
\end{tabular}

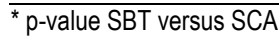

To confirm MSP results, we performed bisulfite sequencing on two methylated and two unmethylated SCAs. The regions B1 (19 CpGs; -192 to -35 relative to TSS) and B2 (24 CpGs; -63 to +154; Figure $2 A$ ), spanning the MSP regions $M 1$ to $M 3$, were investigated. Methylation was not detected in the two unmethylated SCAs, whereas the two methylated SCAs show dense methylation (Figure $2 \mathrm{C}$ ), thereby confirming MSP results. BHMT2 mRNA expression was assessed in 16 FTs from women with ovarian disease, 13 SBTs and 31 SCAs. A 2.5-fold higher mRNA expression was detected in FTs as compared to SBTs $(p=0.015)$ or SCAs $(p=0.002)$ (Figure 3$)$. A correlation between BHMT2 promoter CpG island hypermethylation and mRNA expression was not observed.

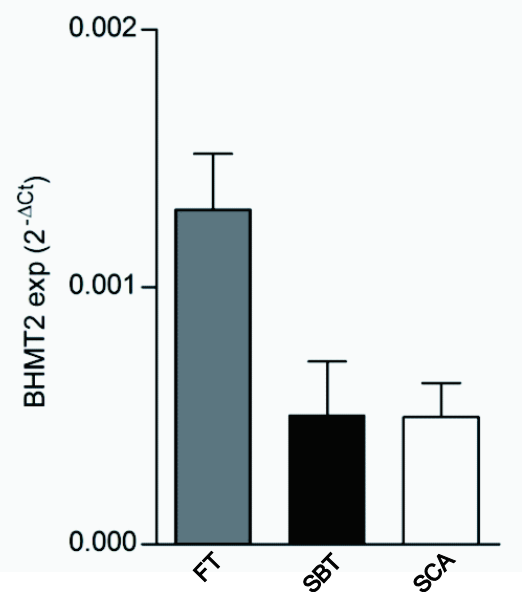

Figure 3: BHMT2 mRNA expression in primary tumors BHMT2 mRNA expression in FTs, SBTs and SCAs. 
Overexpression of BHMT2 reduces colony formation, invasion and migration

To examine if restoration of BHMT2 expression could reduce colony forming capacity, invasion or migration assays using a full-length BHMT2 cDNA expression construct (pcDNA3-BHMT2-HA) or empty vector ( $p$ cDNA3) were performed in SKOV3 cells. The transfected SKOV3 cell line expressed BHMT2 mRNA, whereas BHMT2 protein (predicted mass $\sim 41 \mathrm{kDa}$ ) was not detected (Figure 4A). After two weeks of G418 selection, BHMT2 transfected SKOV3 cells showed a statistically significant $40 \%$ reduction in the number of colonies formed as compared to control cells $(p=0.004$; Figure $4 \mathrm{~B}$ ). The effect of BHMT2 restoration on the invasive ability and motility was studied using a transwell system. As shown in Figure 4C, BHMT2 transfectants showed a strong reduction in invasion through matrigel-coated membranes as compared with control cells $(p=0.036)$, whereas migration was reduced to a lesser extent $(p=0.064$; Figure 4D).

A

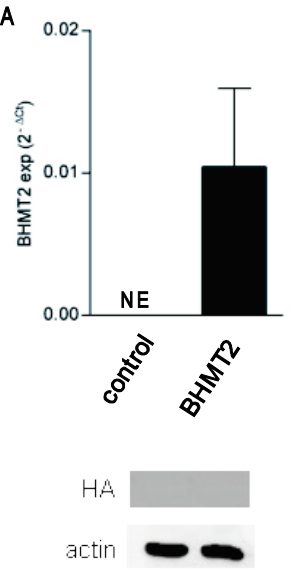

B
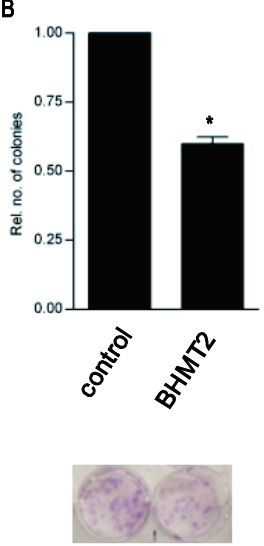

C

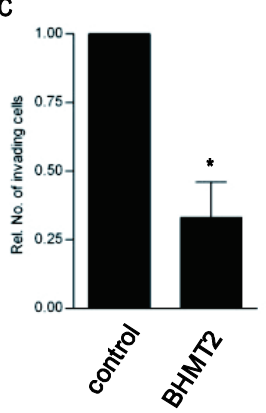

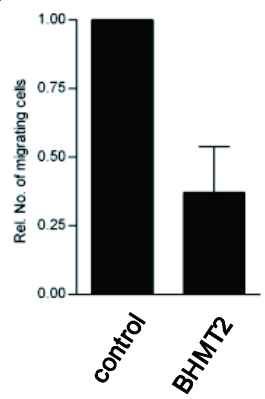

Figure 4: BHMT2 overexpresion reduces colony formation, invasion and migration in SKOV3

(A) BHMT2 mRNA and protein expression in control (empty vector) and BHMT2 transfected SKOV3 cells. Results are plotted as mean values ( \pm SEM) relative to control in three independent experiments performed in duplo, except for SKOV3 control (2 independent experiments). NE: not expressed. (B) Colony formation assay of SKOV3 cells transfected with control or BHMT2 expressing vector, followed by 2 week G418 selection. Panel (C) shows invasion assay of control and BHMT2 transfected cells; measured by counting the number of invaded cells through a matrigel coated transwell. (D) Migration of cells transfected with BHMT2 or control vector through an uncoated transwell system. Quantification of colonies, number of invading and migrating cells is presented as mean values ( \pm SEM) relative to control transfectants of three independent experiments. ${ }^{*} p \leq 0.05$ 


\section{Discussion}

Here we show that BHMT2, present in the TT (see Chapter 5 of this thesis for details) of ovarian cancer cell lines COV504, OVCAR3 and SKOV3, is methylated at the promoter $\mathrm{CPG}$ island in these cell lines. DAC treatment restored BHMT2 mRNA expression, whereas TSA treatment did not induce BHMT2 expression in COV504 and SKOV3, thereby confirming BHMT2 as a TT gene in these cell lines. Although DAC treatment led to massive re-expression in OVCAR3 as well, it was not present in the top tier, since TSA also induced BHMT2 expression in this cell line. We observed that BHMT2 methylation frequencies of histological normal FTs derived from noncancerous patients decreased from region $M 1$ to $M 3$, whereas methylation of SBTs and SCAs did not differ between the regions (Table 2), indicating cancer-specific methylation at region M3. The high frequency of methylation in diseased FTs as compared to FTs derived from non-cancerous patients can be explained by contamination with malignant cells due to macro-dissection (micro-dissection was performed for histologically normal FTs), field effect (i.e. methylation in histological normal tissue adjacent to cancerous processes [17]) or age-related methylation. The mean age of the non-cancerous patients was 31.3 years (range 20-40), and 58.8 years $(26-88)$ for the diseased patients, a difference that was statistically significant $(p<0.001)$. This fact adds to the ongoing discussion on the relevance of choosing the appropriate control tissue in ovarian cancer research. In addition, it is thought that the epithelium of the fallopian tube fimbriae is the site of origin for high-grade SCA [18], which suggests that $B H M T 2$ methylation in these FTs could represent an early disease marker. BHMT2 might be involved in serous ovarian carcinogenesis, through promoter CpG island hypermethylation induced silencing and abrogation of its tumor suppressive properties. This is supported by the fact that loss of heterozygosity (LOH) of 5q13-q14, the locus containing BHMT2, has been detected in up to $46 \%$ of SCAs, whereas LOH was not detected in SBTs $[19,20]$. To test this hypothesis and to further elucidate the functional significance of $B H M T 2$ silencing, colony formation, invasion and migration assays were performed. BHMT2 encodes a methyltransferase that catalyzes the remethylation of homocysteine (Hcy), hereby forming methionine. The BHMT2 protein is highly expressed in human liver and kidney [21]. In COV504, OVCAR3, OV90, and SKOV3 cells transfected with BHMT2, mRNA expression was restored. Expression was 5 to 40 times higher in OV90 cells as compared to the other investigated cell lines. BHMT2 protein was only detected in OV90 transfected cells. This might be explained by instability of the protein. It has been shown previously that the BHMT2 protein rapidly degrades in mammalian cells over-expressing BHMT2 [22]. The considerably higher transcriptional activity in OV90 cells might result in quantities of protein that are sufficient to prevent complete degradation.

$\mathrm{Hcy}$ is an amino acid of the methionine cycle and a well-known player in vascular diseases. High Hcy levels are detected in arteriosclerosis. Proliferation of vascular smooth muscle cells by activation of the MAPK pathway is promoted by $\mathrm{Hcy}[23,24]$. Hcy can activate matrix metallo proteases (MMPs), e.g. MMP2 [25], and is able to remodel the extracellular matrix (ECM) of vessels in animal models [26]. In cancer, epithelial-stromal interactions are of importance as well. Elevated Hcy levels were detected in serum and ascitic fluid of ovarian cancer patients [27, 28]. We 
hypothesized that if BHMT2 is silenced through promoter hypermethylation, remethylation of Hcy to methionine is impaired, resulting in increased levels of Hcy, ECM degradation by MMPs and subsequent invasion. Restoring BHMT2 expression in SKOV3 cells indeed led to reduced colony formation, invasion, and migration (Figures $4 B$ to $4 \mathrm{D}$ respectively). Stromal invasion is the main discriminator between SBTs and SCAs. We previously showed that SerpinA5, a member of the serine protease inhibitor superfamily that inactivates plasma proteases including urokinase-type plasminogen activator (UPA), is differentially expressed between SBTs and SCAs. SCAs show loss of SerpinA5 mRNA and protein expression as compared to SBTs $[29,30]$. Along similar lines, methylated BHMT2 may represent an additional tumor invasion related biomarker for serous ovarian cancer.

In summary, we have shown that BHMT2 is frequently methylated in serous ovarian tumors. To our knowledge, this is the first study reporting epigenetic inactivation of BHMT2 in human (ovarian) cancer. Since BHMT2 restoration decreased colony formation and suppressed invasion and migration, BHMT2 might be a novel candidate tumor suppressor gene in serous ovarian cancer. 


\section{References}

1. Cannistra SA. Cancer of the ovary. N Engl J Med 2004;351(24):2519-29.

2. Ferlay J, Shin HR, Bray F, Forman D, Mathers C, Parkin DM. Estimates of worldwide burden of cancer in 2008: GLOBOCAN 2008. Int J Cancer 2010;127(12):2918-27.

3. Prat J. Surface epithelial-stromal tumors: Serous tumors: Saunders; 2004: 83-111

4. Crum CP, Drapkin R, Miron A, et al. The distal fallopian tube: a new model for pelvic serous carcinogenesis. Curr Opin Obstet Gynecol 2007;19(1):3-9.

5. Kurman RJ, Shih le M. Pathogenesis of ovarian cancer: lessons from morphology and molecular biology and their clinical implications. Int J Gynecol Pathol 2008;27(2):151-60.

6. Marquez RT, Baggerly KA, Patterson AP, et al. Patterns of gene expression in different histotypes of epithelial ovarian cancer correlate with those in normal fallopian tube, endometrium, and colon. Clin Cancer Res 2005;11(17):6116-26.

7. Massuger L, Roelofsen $T$, Ham M, Bulten J. The origin of serous ovarian cancer may be found in the uterus: a novel hypothesis. Medical hypotheses 2010;74(5):859-61.

8. Crispens MA. Borderline ovarian tumours: a review of the recent literature. Curr Opin Obstet Gynecol 2003;15(1):39-43.

9. Sieben NL, Macropoulos P, Roemen GM, et al. In ovarian neoplasms, BRAF, but not KRAS, mutations are restricted to low-grade serous tumours. J Pathol 2004;202(3):336-40.

10. Singer $G$, Oldt $R, 3 r d$, Cohen $Y$, et al. Mutations in BRAF and KRAS characterize the development of low-grade ovarian serous carcinoma. J Natl Cancer Inst 2003;95(6):484-6.

11. Shih le M, Kurman RJ. Molecular pathogenesis of ovarian borderline tumors: new insights and old challenges. Clin Cancer Res 2005;11(20):7273-9.

12. Salani R, Kurman RJ, Giuntoli R, 2nd, et al. Assessment of TP53 mutation using purified tissue samples of ovarian serous carcinomas reveals a higher mutation rate than previously reported and does not correlate with drug resistance. Int J Gynecol Cancer 2008;18(3):48791.

13. Derks S, Lentjes MH, Hellebrekers DM, de Bruine AP, Herman JG, van Engeland $M$. Methylation-specific PCR unraveled. Cell Oncol 2004;26(5-6):291-9.

14. Herman JG, Graff JR, Myohanen S, Nelkin BD, Baylin SB. Methylation-specific PCR: a novel PCR assay for methylation status of CpG islands. Proceedings of the National Academy of Sciences of the United States of America 1996;93(18):9821-6.

15. van Engeland M, Weijenberg MP, Roemen GM, et al. Effects of dietary folate and alcohol intake on promoter methylation in sporadic colorectal cancer: the Netherlands cohort study on diet and cancer. Cancer research 2003;63(12):3133-7.

16. De Meyer T, Bijsmans I, Van de Vijver K, et al. E2Fs mediate a fundamental cell-cycle deregulation in high-grade serous ovarian carcinomas. J Pathol 2008;217(1):14-20.

17. Slaughter DP, Southwick HW, Smejkal W. Field cancerization in oral stratified squamous epithelium; clinical implications of multicentric origin. Cancer 1953;6(5):963-8.

18. Levanon K, Crum C, Drapkin R. New insights into the pathogenesis of serous ovarian cancer and its clinical impact. J Clin Oncol 2008;26(32):5284-93.

19. Wang VW, Bell DA, Berkowitz RS, Mok SC. Whole genome amplification and highthroughput allelotyping identified five distinct deletion regions on chromosomes 5 and 6 in microdissected early-stage ovarian tumors. Cancer research 2001;61(10):4169-74.

20. Tavassoli M, Steingrimsdottir H, Pierce E, et al. Loss of heterozygosity on chromosome $5 q$ in ovarian cancer is frequently accompanied by TP53 mutation and identifies a tumour suppressor gene locus at 5q13.1-21. Br J Cancer 1996;74(1):115-9.

21. Chadwick LH, McCandless SE, Silverman GL, Schwartz S, Westaway D, Nadeau JH. Betainehomocysteine methyltransferase-2: cDNA cloning, gene sequence, physical mapping, and expression of the human and mouse genes. Genomics 2000;70(1):66-73. 
22. Li $\mathrm{F}$, Feng $\mathrm{Q}$, Lee $\mathrm{C}$, et al. Human betaine-homocysteine methyltransferase (BHMT) and BHMT2: common gene sequence variation and functional characterization. Molecular genetics and metabolism 2008;94(3):326-35.

23. Brown JC, Rosenquist TH, Monaghan DT. ERK2 activation by homocysteine in vascular smooth muscle cells. Biochemical and biophysical research communications 1998;251(3):669-76.

24. Woo DK, Dudrick SJ, Sumpio BE. Homocysteine stimulates MAP kinase in bovine aortic smooth muscle cells. Surgery 2000;128(1):59-66.

25. Bescond A, Augier T, Chareyre C, Garcon D, Hornebeck W, Charpiot P. Influence of homocysteine on matrix metalloproteinase-2: activation and activity. Biochemical and biophysical research communications 1999;263(2):498-503.

26. Tyagi SC. Homocyst(e)ine and heart disease: pathophysiology of extracellular matrix. Clin Exp Hypertens 1999;21(3):181-98.

27. Corona G, Toffoli G, Fabris $M$, et al. Homocysteine accumulation in human ovarian carcinoma ascitic/cystic fluids possibly caused by metabolic alteration of the methionine cycle in ovarian carcinoma cells. Eur J Cancer 1997;33(8):1284-90.

28. Wu LL, Wu JT. Hyperhomocysteinemia is a risk factor for cancer and a new potential tumor marker. Clin Chim Acta 2002;322(1-2):21-8.

29. Bijsmans IT, Smits KM, de Graeff $P$, et al. Loss of SerpinA5 protein expression is associated with advanced-stage serous ovarian tumors. Mod Pathol 2011;24(3):463-70.

30. Sieben NL, Oosting J, Flanagan AM, et al. Differential gene expression in ovarian tumors reveals Dusp 4 and Serpina 5 as key regulators for benign behavior of serous borderline tumors. J Clin Oncol 2005;23(29):7257-64. 
Epigenetic silencing of BHMT2 in serous ovarian cancer

Insert supplementary table 1 


\section{CHAPTER 7}

General discussion 


\section{Defining the borderline}

The concept of ovarian borderline tumors has been controversial for over a century, with a first report originating from 1898 describing papillary ovarian cystadenomas with "clinical features that stand on the border of malignancy". This was followed by numerous publications describing tumors appearing to be "intermediate" between benign cystadenomas and malignant carcinomas [1]. In the 1950s and 1960s, the modern history of borderline tumors started when researchers became aware of the fact that a subset of carcinomas had a significantly better prognosis, even when presented at advanced stage [2]. In 1973, the World Health Organization (WHO) created a separate category of ovarian tumors and designated these "borderline" or "tumor of low malignant potential (LMP)" [1, 2] characterized by lacking obvious stromal invasion. Mitotic activity and nuclear abnormalities are intermediate between clearly benign and unquestionable malignant tumors of a similar cell. The model and terminology for borderline tumors continued to be challenged. The "dualistic ovarian cancer development model" is cited by many researchers, indicating that this model is currently the most appreciated model [3]. Despite the current consensus on the model of ovarian cancer development, the biology of serous borderline tumors (SBTs) and serous carcinomas (SCAs) is not completely understood yet. Multiple hypotheses in the field exist. Some researchers believe that SBTs may progress to SCAs, whereas others favor the hypothesis that these tumors are distinct entities which develop via different pathways. Since the distinction between SBTs and SCAs is important from a clinical point of view as both patient management and prognosis differ considerably, it is important to unravel the biology underlying the development of serous borderline tumors and carcinomas.

\section{Does a benign-borderline-carcinoma sequence exist in serous ovarian tumors?}

It remains unclear whether a borderline tumor is an ovarian carcinoma precursor or represents a separate entity. In 1996, Link et al. suggested a model in which the evolution of ovarian tumors is shown as a result of genetic and environmental factors via progression from normal ovarian surface epithelium to benign tumor to borderline tumor to low-grade carcinoma to high-grade ovarian carcinoma [4]. Loss of chromosomal arm $6 \mathrm{q}$ also favored for the progression from benign ovarian cysts to borderline to invasive carcinoma [5]. Shih and Kurman [3] proposed a dualistic ovarian cancer development model in which surface epithelium tumors are divided in type I and type II tumors. Low-grade SCAs (LG-SCAs) develop in a stepwise manner from SBTs or atypical proliferative serous tumors (APSTs) through micropapillary serous carcinomas (MPSCs) prior to becoming invasive and are designated type I tumors, whereas type II tumors are high-grade SCAs (HG-SCAs) which develop de novo. Evidence is emerging that LG-SCAs (grade 1; SCA1s) have more in common with SBTs than with HG-SCAs (grade 2/3; SCA2/SCA3s) [6, 7]. Since ovarian cancer is mostly diagnosed at an advanced stage and grade, SCA1s are not frequently identified. The data presented in Chapters 2, 3, and 5 support earlier findings that LG-SCAs are more similar to SBTs. SerpinA5 protein expression was significantly higher in LG-SCAs as compared to HG-SCAs, whereas no difference was detected between advanced-stage SBTs and LG-SCAs [8]. We also showed that TP53 mutations are frequently detected in SCA3s, less often in SCA1s, and absent in SBTs. E2F target gene expression patterns of 
SCA1s were more similar to SBTs, whereas SBTs and SCA3s differ to a greater extent [9]. BHMT2 promoter CpG island hypermethylation increased from SBT to LG-SCA to HG-SCA. Although the difference was not statistically significant due to the very small number of analyzed SCA1s, these data favor the hypothesis that SBTs are unrelated to HG-SCAs. It will remain difficult to answer the question whether SBTs can develop into LG-SCAs since the number of available SCA1s is low and SBTs always will be excised upon diagnosis.

\section{Invasion: the main discriminator between SBTs and SCAs}

The main histological discriminator between SBTs and SCAs is the presence of destructive stromal invasion in SCAs $[10,11]$. In this thesis we present additional molecular evidence that invasion is indeed important in the discrimination between these tumor entities. First, SerpinA5, a candidate tumor suppressor gene (TSG) that inhibits uPA activation [12] was analyzed by immunohistochemistry in SBTs and SCAs [8]. When SerpinA5 protein expression is downregulated or lost, expression of UPA and activation of the downstream target MMP9 leads to extracellular matrix (ECM) degradation, invasion of tumor cells into the surrounding tissue, and increased metastatic potential $[8,13,14]$. SerpinA5 protein expression was reduced in advancedstage HG-SCAs and in omental metastases. An interesting finding was that advancedstage SBTs expressed SerpinA5 at significantly lower levels than early-stage SBTs. Review of the corresponding $\mathrm{H} \& \mathrm{E}$ slides showed that the majority of the advancedstage SBTs harbored microinvasive and/or micropapillary features, what might be associated with increased aggressiveness of the tumor. Downregulation of SerpinA5 protein expression in advanced-stage SBTs was unexpected, however these data fit with the observation that these SBTs are closely related to LG-SCAs. Thus, loss or reduction of SerpinA5 protein expression seems to be associated with invasion. In a diagnostic setting, lowered SerpinA5 protein expression in SBTs might have prognostic significance. It is recommended to perform a complete staging procedure in these cases since microinvasive growth is often associated with invasive implants [15]. Second, four of five genes (MECOM, LIMK1, ACTN4, and NCOA3) identified by decision tree analysis of MLPA copy number data have been described to be involved in migration and invasion. Downregulation of the putative TSG SerpinA5 and overexpression of the oncogene LIMK1 in SCAs can induce UPA activation and subsequent destructive invasion, a phenomenon not seen in SBTs. Interplay between pathways has also been shown for NCOA3 and E2F1 [16]. Whether copy number gain or amplification of these genes actually is associated with overexpression remains to be determined. It has recently been described that combined copy number gain and promoter CpG island hypermethylation can occur at certain chromosomal regions [17]. Although it is unclear whether these events occur at the same time, epigenetic inactivation of TSGs might serve to counteract the effect of copy number gains driven by other genes within the amplicon [17]. Third, we hypothesize that downregulation of BHMT2 due to promoter $\mathrm{CpG}$ island hypermethylation results in elevated levels of homocysteine, which in turn has an effect on MMPs and ECM degradation, followed by invasion $[18,19]$. It has been shown that homocysteine levels are elevated in serum and ascites of ovarian cancer patients [20, 21]. Unfortunately, these data are unavailable for our study population but our preliminary data warrant further 
investigation. Taken together, data presented in this thesis support the histological evidence that invasion is the main discriminator between SBTs and SCAs.

\section{Clinical relevance}

Since both histological and molecular evidence indicate that invasion is the main discriminator between SBTs and SCAs, inhibition of invasion could be an attractive cancer treatment strategy. The mechanism(s) responsible for the downregulation of SerpinA5 in advanced-stage SBTs and SCAs are currently unknown. We performed a pilot sequencing study, however mutations were not identified (data not shown). Since SerpinA5 does not contain a CPG island (using stringent criteria), hypermethylation of the promoter CpG island seems unlikely. SerpinA5 is located at chromosome 14q32.1. Chromosomal loss of $14 q$ has been described in approximately $50 \%$ of ovarian tumors, irrespective of histological subtype $[22,23]$, indicating that loss of heterozygosity (LOH) might be responsible for SerpinA5 downregulation in half of the cases. Reintroduction of the gene using in vitro and in vivo experiments might reverse the invasive and metastatic phenotype, as has been shown previously in breast carcinomas [24]. To test whether SerpinA5 is indeed a novel TSG, the tumor suppressive characteristics have to be confirmed in animal models. Inactivation of the oncogenes involved in invasion might be accomplished using neutralizing antibodies that specifically bind and block oncogenic expression. Several in vitro and in vivo studies have been successful in reducing invasion using neutralizing antibodies targeting the TGF $\beta$ signaling pathway $[25,26]$ or inhibition of UPA and MMP9 mRNA expression in ovarian cancer cell lines using an antibody directed against CXCR4, a downstream target of NFKB [27]. To date, clinical trials using these antibodies are not yet being performed for ovarian cancer patients (www.clinicaltrials.gov). LIMK expression has been shown to be inhibited in vitro using small molecule LIMK inhibitors [28]. NCOA3 has recently been described as a therapeutic target for lung cancer since its downregulation resulted in increased sensitivity for the chemotherapeutic regimen gefitinib [29]. A screen for MECOM expression in chronic-phase CML patients suggested that at the time of imatinib failure expression may predict for response to second-line tyrosine kinase inhibitor therapy and consequently aid clinical management [30]. Demethylating agents might be able to reverse the epigenetically silenced status of BHMT2 resulting in re-expression of this gene, subsequent downregulation of homocysteine and thus inhibition of MMP expression, ECM degradation and invasion.

Promoter CPG island hypermethylation in serous ovarian tumors: opportunities and threats

Serous ovarian cancer epigenetics has not been studied extensively. As described previously [31, 32], this thesis shows that serous ovarian tumors have a unique promoter $\mathrm{CpG}$ island hypermethylation profile as compared to other solid tumors, e.g. breast, kidney and colorectal tumors (data not shown). In addition, the methylation frequency of candidate genes was low (defined as less than 35\%; most candidates showed even lower than $15 \%$ promoter CpG island hypermethylation) in these tumors, which is in accordance with another study analyzing ovarian cancer [31]. BHMT2 was identified as a putative TSG silenced by promoter CpG island hypermethylation in SBTs and SCAs. Overexpression of BHMT2 in in vitro assays showed decreased invasion and 
migration of the ovarian cancer cell line SKOV3. To determine the tumor suppressive characteristics of BHMT2 in vivo, animal models need to be analyzed.

The genes identified in the genome wide pharmacological unmasking approach were infrequently methylated in primary tumors. Evidence is emerging that $\mathrm{CpG}$ island hypermethylation can vary significantly between different positions in the CpG island. In the performed studies, methylation specific PCR (MSP) was used to determine hypermethylation. By using this technique, only a restricted region of the CpG island is analyzed. In addition, evidence is emerging that $\mathrm{CpG}$ methylation can occur outside the classical CpG islands, in the so-called CpG shores [33]. CpG shores are defined as regions with a relatively low $\mathrm{CpG}$ density located within $2 \mathrm{~kb}$ of traditional $\mathrm{CpG}$ islands. Aberrant methylation in these shores was reported to separate tissue subtypes and cancerous tissue from matched normal tissue. This observation changes the current idea from studying exclusively $\mathrm{CpG}$ islands in promoter regions to much larger regions of interest, which potentially possess regulatory regions previously characterized. Using next generation sequencing technologies, such as MBD affinity genome sequencing [34, 35] or genome-wide bisulfite sequencing accurate and detailed information of the entire serous ovarian cancer epigenome will hopefully become available soon.

DNA promoter hypermethylation changes have implications for early cancer detection, prognosis and treatment. CA125, currently the most used serum marker for detection of ovarian cancer is not very specific since this marker is elevated in $>80 \%$ of advanced stage patients, in $~ 50 \%$ of stage I cancers, and is undetectable in mucinous tumors [3638]. Another drawback of CA125 is that serum concentrations can also be elevated in benign conditions, such as endometriosis, congestive heart failure or cirrhosis [39]. Thus, there is a need for novel specific (early detection) markers. Aberrant promoter $\mathrm{CpG}$ island hypermethylation is frequently observed in cancer development and is considered to be one of the first molecular changes in tumorigenesis [40], indicating that alterations in the epigenetic profile might be used for early detection. It has been shown that hypermethylated genes can be detected via minimally invasive ways in serum, plasma and peritoneal fluid of ovarian cancer patients [41]. However, thus far, not one single gene has been identified that is hypermethylated in a large proportion of ovarian tumors. A panel of methylated genes will be required to detect ovarian cancer, or more specific, each histological subtype, with sufficient specificity and sensitivity. It is of interest to determine the methylation profile of control subjects using the genes analyzed in our studies to establish such a marker panel. Promoter CpG island hypermethylation might also be used as a diagnostic indicator. The differential diagnosis of SBTs and SCAs might be problematic. In our study, CDO1 was identified as differentially hypermethylated between SBTs and SCAs (10\% versus $66 \%$ ), indicating that $C D O 1$ promoter hypermethylation might serve as a diagnostic marker. To validate the use of $C D O 1$ hypermethylation as a diagnostic marker in serous ovarian tumors, larger independent studies are required. Analysis of this methylation marker together with the described MLPA marker panel might even increase correct tumor classification. Further analysis of our re-expression data in combination with e.g. MBD affinity genome sequencing might reveal sensitive panels for diagnostic, early detection and/or therapeutic purposes. 
Epigenetic therapy, defined as reversing epigenetic alterations in tumor cells, is a new strategy for cancer treatment. Several agents have been discovered that inhibit promoter CpG island hypermethylation (DNMT inhibitors) or histone deacetylation (HDAC inhibitors). Good results have been booked for DNMT inhibitors in patients with myelodysplastic syndrome [42-44]. The effect of these inhibitors on ovarian tumors is currently under investigation in clinical trials (www.clinicaltrials.gov). The methylation markers described in this thesis might be used as markers to determine whether DNMT inhibitors indeed induce demethylation of critical genes in serous ovarian tumors. Since some of the epigenetically silenced TSGs may exhibit invasion inhibiting characteristics, anti-invasion effects of demethylating agents and deacetylation inhibitors might be expected. However, at present, the drugs that target epigenetic alterations are rather nonspecific. Thus, there is need for specific small-molecule DNMT and HDAC inhibitors [45].

\section{Conclusions and future perspectives}

All results presented in the present thesis need to be validated in independent prospective and retrospective populations before the tests can be introduced in clinical practice for trials and/or as a diagnostic tool. To cite J. Ioannidis: "There is increasing concern that most current published research findings are false". The probability that a research claim is indeed correct depends on several factors, e.g. the statistical power of the study, bias, the number of other studies answering the same research question, and analytical methods [46]. Thus, for future studies, the following issues should be taken into account: 1) ovarian cancer is a heterogeneous disease and the various subtypes should be considered as separate diseases so one is able to compare data from independent studies; 2) the study design, i.e. experimental methodology (including among others tissue preparation, primer location, microarray platforms, bioinformatics tools, quality control, and statistical power) should be appropriate and comparable among studies; 3) the source of control tissue (e.g. ovarian or fallopian tube tissue, short-term culture of ovarian surface epithelium, immortalized ovarian surface epithelium cell lines) should also be carefully addressed and uniform, allowing data comparison; and 4) the used terminology should be uniform. Currently, the definition of low-grade and high-grade carcinoma varies between research groups. To correctly classify serous tumors, agreement needs to be

reached. It is obvious that multi-centre collaborations are required to be able to analyze sufficient numbers of tumors and subsequently draw powerful conclusions. Eventually, knowledge of molecular mechanisms involved in serous ovarian carcinogenesis will lead to the identification of prognostic and predictive markers that can be used to select patients likely to benefit from different treatment strategies. 


\section{References}

1. Hart WR. Borderline epithelial tumors of the ovary. Mod Pathol 2005;18 Suppl 2:S33-50.

2. Kurman RJ, Seidman JD, Shih IM. Serous borderline tumours of the ovary. Histopathology 2005;47(3):310-5.

3. Shih le M, Kurman RJ. Ovarian tumorigenesis: a proposed model based on morphological and molecular genetic analysis. Am J Pathol 2004;164(5):1511-8.

4. Link CJ, Jr., Kohn E, Reed E. The relationship between borderline ovarian tumors and epithelial ovarian carcinoma: epidemiologic, pathologic, and molecular aspects. Gynecologic oncology 1996;60(3):347-54.

5. Tibiletti MG, Bernasconi B, Furlan D, et al. Chromosome 6 abnormalities in ovarian surface epithelial tumors of borderline malignancy suggest a genetic continuum in the progression model of ovarian neoplasms. Clin Cancer Res 2001;7(11):3404-9.

6. Bonome T, Lee JY, Park DC, et al. Expression profiling of serous low malignant potential, low-grade, and high-grade tumors of the ovary. Cancer research 2005;65(22):10602-12.

7. Meinhold-Heerlein I, Bauerschlag D, Hilpert F, et al. Molecular and prognostic distinction between serous ovarian carcinomas of varying grade and malignant potential. Oncogene 2005;24(6):1053-65.

8. Bijsmans IT, Smits KM, de Graeff $P$, et al. Loss of SerpinA5 protein expression is associated with advanced-stage serous ovarian tumors. Mod Pathol 2011;24(3):463-70.

9. De Meyer T, Bijsmans IT, Van de Vijver KK, et al. E2Fs mediate a fundamental cell-cycle deregulation in high-grade serous ovarian carcinomas. J Pathol 2009;217(1):14-20.

10. Cannistra SA. Cancer of the ovary. N Engl J Med 2004;351(24):2519-29.

11. Prat J. Surface epithelial-stromal tumors: Serous tumors: Saunders; 2004: 83-111

12. Geiger M. Protein $C$ inhibitor, a serpin with functions in- and outside vascular biology. Thrombosis and haemostasis 2007;97(3):343-7.

13. Wakita T, Hayashi T, Nishioka J, et al. Regulation of carcinoma cell invasion by protein C inhibitor whose expression is decreased in renal cell carcinoma. International journal of cancer 2004;108(4):516-23.

14. Cao Y, Becker C, Lundwall A, et al. Expression of protein $\mathrm{C}$ inhibitor (PCl) in benign and malignant prostatic tissues. The Prostate 2003;57(3):196-204.

15. McKenney JK, Balzer BL, Longacre TA. Patterns of stromal invasion in ovarian serous tumors of low malignant potential (borderline tumors): a reevaluation of the concept of stromal microinvasion. The American journal of surgical pathology 2006;30(10):1209-21.

16. Mussi P, Yu C, O'Malley BW, Xu J. Stimulation of steroid receptor coactivator-3 (SRC-3) gene overexpression by a positive regulatory loop of E2F1 and SRC-3. Molecular endocrinology (Baltimore, Md 2006;20(12):3105-19.

17. van Engeland M, Derks S, Smits KM, Meijer GA, Herman JG. Colorectal cancer epigenetics: complex simplicity. J Clin Oncol 2011;29(10):1382-91.

18. Bescond A, Augier T, Chareyre C, Garcon D, Hornebeck W, Charpiot P. Influence of homocysteine on matrix metalloproteinase-2: activation and activity. Biochemical and biophysical research communications 1999;263(2):498-503.

19. Tyagi SC. Homocyst(e)ine and heart disease: pathophysiology of extracellular matrix. Clin Exp Hypertens 1999;21(3):181-98.

20. Corona G, Toffoli G, Fabris $M$, et al. Homocysteine accumulation in human ovarian carcinoma ascitic/cystic fluids possibly caused by metabolic alteration of the methionine cycle in ovarian carcinoma cells. Eur J Cancer 1997;33(8):1284-90.

21. Wu LL, Wu JT. Hyperhomocysteinemia is a risk factor for cancer and a new potential tumor marker. Clinica chimica acta; international journal of clinical chemistry 2002;322(1-2):21-8. 
22. Bandera $\mathrm{CA}$, Takahashi $\mathrm{H}$, Behbakht $\mathrm{K}$, et al. Deletion mapping of two potential chromosome 14 tumor suppressor gene loci in ovarian carcinoma. Cancer research 1997;57(3):513-5.

23. Cliby W, Ritland S, Hartmann L, et al. Human epithelial ovarian cancer allelotype. Cancer research 1993;53(10 Suppl):2393-8.

24. Asanuma K, Yoshikawa T, Hayashi T, et al. Protein C inhibitor inhibits breast cancer cell growth, metastasis and angiogenesis independently of its protease inhibitory activity. International journal of cancer 2007;121(5):955-65.

25. Ganapathy V, Ge R, Grazioli A, et al. Targeting the Transforming Growth Factor-beta pathway inhibits human basal-like breast cancer metastasis. Molecular cancer 2010;26(9):122-37.

26. Zhong Z, Carroll KD, Policarpio D, et al. Anti-transforming growth factor beta receptor II antibody has therapeutic efficacy against primary tumor growth and metastasis through multieffects on cancer, stroma, and immune cells. Clin Cancer Res 2010;16(4):1191-205.

27. Miyanishi N, Suzuki Y, Simizu S, Kuwabara Y, Banno K, Umezawa K. Involvement of autocrine CXCL12/CXCR4 system in the regulation of ovarian carcinoma cell invasion. Biochemical and biophysical research communications 2010;403(1):154-9.

28. Manetti F. LIM kinases are attractive targets with many macromolecular partners and only a few small molecule regulators. Medicinal research reviews 2011.

29. Cai D, Shames DS, Raso MG, et al. Steroid receptor coactivator-3 expression in lung cancer and its role in the regulation of cancer cell survival and proliferation. Cancer research 2010;70(16):6477-85.

30. Daghistani M, Marin D, Khorashad JS, et al. EVI-1 oncogene expression predicts survival in chronic-phase CML patients resistant to imatinib treated with second-generation tyrosine kinase inhibitors. Blood 2010;116(26):6014-7.

31. Esteller M, Corn PG, Baylin SB, Herman JG. A gene hypermethylation profile of human cancer. Cancer research 2001;61(8):3225-9.

32. Costello JF, Fruhwald MC, Smiraglia DJ, et al. Aberrant CpG-island methylation has nonrandom and tumour-type-specific patterns. Nature genetics 2000;24(2):132-8.

33. Irizarry RA, Ladd-Acosta C, Wen B, et al. The human colon cancer methylome shows similar hypo- and hypermethylation at conserved tissue-specific $\mathrm{CpG}$ island shores. Nature genetics 2009;41(2):178-86.

34. Serre D, Lee BH, Ting AH. MBD-isolated Genome Sequencing provides a high-throughput and comprehensive survey of DNA methylation in the human genome. Nucleic acids research 2010;38(2):391-9.

35. Laird PW. Principles and challenges of genome-wide DNA methylation analysis. Nature reviews 2010;11(3):191-203.

36. Kozak KR, Su F, Whitelegge JP, Faull K, Reddy S, Farias-Eisner R. Characterization of serum biomarkers for detection of early stage ovarian cancer. Proteomics 2005;5(17):4589-96.

37. Gagnon A, Kim JH, Schorge JO, et al. Use of a combination of approaches to identify and validate relevant tumor-associated antigens and their corresponding autoantibodies in ovarian cancer patients. Clin Cancer Res 2008;14(3):764-71.

38. Nustad K, Bast RC, Jr., Brien TJ, et al. Specificity and affinity of 26 monoclonal antibodies against the CA 125 antigen: first report from the ISOBM TD-1 workshop. International Society for Oncodevelopmental Biology and Medicine. Tumour Biol 1996;17(4):196-219.

39. Moore RG, Maclaughlan S. Current clinical use of biomarkers for epithelial ovarian cancer. Current opinion in oncology;22(5):492-7.

40. Jones PA, Baylin SB. The fundamental role of epigenetic events in cancer. Nature reviews 2002;3(6):415-28. 
41. Ibanez de Caceres I, Battagli C, Esteller M, et al. Tumor cell-specific BRCA1 and RASSF1A hypermethylation in serum, plasma, and peritoneal fluid from ovarian cancer patients. Cancer research 2004;64(18):6476-81.

42. Santos FP, Kantarjian H, Garcia-Manero G, Issa JP, Ravandi F. Decitabine in the treatment of myelodysplastic syndromes. Expert review of anticancer therapy 2010;10(1):9-22.

43. Issa JP. Epigenetic changes in the myelodysplastic syndrome. Hematology/oncology clinics of North America 2010;24(2):317-30.

44. Shen L, Kantarjian $H$, Guo $Y$, et al. DNA methylation predicts survival and response to therapy in patients with myelodysplastic syndromes. J Clin Oncol 2010;28(4):605-13.

45. Tuma RS. Targeted epigenetic therapies: the next frontier? Journal of the National Cancer Institute 2010;102(24):1824-5.

46. Ioannidis JP. Why most published research findings are false. PLoS medicine 2005;2(8):e124. 
SUMMARY 
Ovarian cancer is a leading cause of cancer-related deaths among women with gynecologic malignancies. Long-term survival rates range from $10-30 \%$ for advanced disease, to $80-90 \%$ for early-stage disease and are above $90 \%$ for borderline tumors. The high mortality is related to the fact that in most cases the disease has already spread to the upper abdomen at time of presentation because women generally do not have complaints. The biology of serous borderline tumors (SBTs) and serous carcinomas (SCAs) is not completely understood yet. Multiple hypotheses for tumor development exist, either favoring a progression model or believing that these tumors are distinct entities.

The aim of this thesis was to investigate whether SBTs can be distinguished from SCAs. Since the distinction between SBTs and SCAs is important from a clinical point of view as both patient management and prognosis differ considerably, it is important to unravel the underlying biological mechanisms. Additional tools to assist pathologists in making the differential diagnosis could aid better tumor classification. Immunohistochemical and molecular biological approaches were performed in this respect.

Our previous microarray study revealed that although the MAPK pathway is activated in SBTs, activation of downstream genes involved in extracellular matrix (ECM) degradation is absent, suggesting an uncoupling of these events. SerpinA5, an urokinase Plasminogen Activator-inhibitor, a key regulator for indolent borderline behavior, was identified in this study. Since SCAs are characterized by loss of SerpinA5 mRNA expression, we hypothesized that SerpinA5 protein expression is reduced or lost in SCAs as compared to SBTs. To address this hypothesis, SerpinA5 protein expression was determined by immunohistochemistry in Chapter 2. Reduced or absent SerpinA5 protein staining was observed in SCAs as compared to SBTs. To validate these results, loss of SerpinA5 protein expression was performed in a larger, independent patient population, also including omental metastases. SerpinA5 protein expression was significantly lowered in the omental metastases when compared to the matching primary SCA. Interestingly, SerpinA5 protein expression was reduced in advancedstage SBTs, often characterized by micropapillary growth and/or microinvasion, when compared to early-stage SBTs, indicating that SerpinA5 loss is associated with more aggressive tumors. Further microarray data exploration to identify molecular differences between SBTs and SCAs is described in Chapter 3. Significance analysis of microarrays (SAM) and Ingenuity Pathway analysis revealed central roles for E2Fs and CDKN1A (p21) in SCA cell cycle deregulation. A significant increase in mRNA expression of the transcription factors E2F1 and E2F3, and their target genes was observed in SCAs as compared to SBTs, whereas the cell cycle inhibitor CDKN1A was upregulated in SBTs. E2Fs were identified as fundamental cell cycle deregulators in high-grade SCAs (HG-SCAs). These data show that SBTs and SCAs are different entities since the cell cycle progression pathway is activated in SCAs, while inhibited in SBTs. In Chapter 4, we investigated if SBTs and SCAs can be distinguished from each other based on DNA copy number alterations (CNAs) as determined by multiplex ligation-dependent probe amplification (MLPA) analysis. SCAs contained significantly more CNAs as compared to SBTs. Unsupervised hierarchical clustering of CNAs showed that the majority of SBTs 
and SCAs clustered separately, supporting the hypothesis that SBTs and HG-SCAs are unrelated entities. Moreover, decision tree analysis based on MLPA data was performed to investigate whether a more accurate prediction of SBTs and SCAs could be achieved. Gain of MDS1 and EVI1 complex locus (MECOM), LIM domain Kinase 1 (LIMK1), NFKB Inhibitor Epsilon (NFKBIE) and Nuclear Receptor Coactivator 3 (NCOA3) and loss of Actinin alpha 4 (ACTN4) predicted correctly whether a tumor was a SBT or SCA in 83/86 (96.5\%) cases. These data indicate that analysis of CNAs of these 5 key genes may be used for molecular classification of SBTs and SCAs thereby assisting pathologists in making the differential diagnosis. Since PCR-based techniques are relatively easy to implement in molecular diagnostic settings, ACTN4, MECOM, NCOA3, and NFKBIE might be used as diagnostic markers in the discrimination between SBTs and SCAs since these genes were only altered in SCAs. In addition to genetic alterations, epigenetic mechanisms frequently alter gene expression. Promoter $\mathrm{CpG}$ island hypermethylation of tumor suppressor genes (TSGs) is the best characterized and most studied epigenetic alteration in cancer. However, only a few studies investigating epigenetic silencing in serous ovarian tumors have been published. The search for novel hypermethylated TSGs in serous ovarian tumors able to discriminate between SBTs and SCAs are described in Chapter 5. Three approaches were used: 1) a candidate gene approach (literature-based genes reported to be frequently methylated in other cancers); 2) promoter sequence (genome-wide promoter alignment in combination with specific sequence motifs in the promoter region of CpG islands); and 3) pharmacological unmasking of hypermethylated TSGs to reactivate transcriptional silenced genes. Overall, our results indicated that promoter CpG island hypermethylation of candidate markers is not a frequently occurring event in serous ovarian tumors. Betaine-homocysteine methyltransferase 2 (BHMT2) and cysteine dioxygenase type I (CDO1) were identified as novel markers differentially methylated between SBTs and SCAs. We showed cancer-specific BHMT2 promoter CpG island hypermethylation at the region downstream of the transcription start site (Chapter 6). The in vitro tumor suppressive properties of BHMT2, a methyltransferase catalyzing the remethylation of homocysteine to methionine, were investigated in Chapter 6 using an ovarian cancer cell line in which we restored BHMT2 expression by overexpression. Restoration of $B H M T 2$ reduced colony formation, invasion and migration, indicating that $B H M T 2$ is a novel candidate tumor suppressor gene in vitro in serous ovarian cancer. The general discussion in Chapter 7 summarizes the major findings of the studies presented in this thesis, followed by suggestions for diagnostic and clinical applications of the identified genes and future directions.

In conclusion, we identified several markers able to discriminate between SBTs and SCAs. However, these novel genes need to be validated in independent prospective and retrospective populations before the tests can be introduced in clinical practice for trials and/or as a diagnostic tool. In the end, detailed information about molecular mechanisms involved in serous ovarian carcinogenesis will lead to the identification of prognostic and predictive markers that can be used to select patients likely to benefit from different treatment strategies. Hopefully, (epi)genomes of individual patients will become available to direct personalized medicine of ovarian serous tumors. 


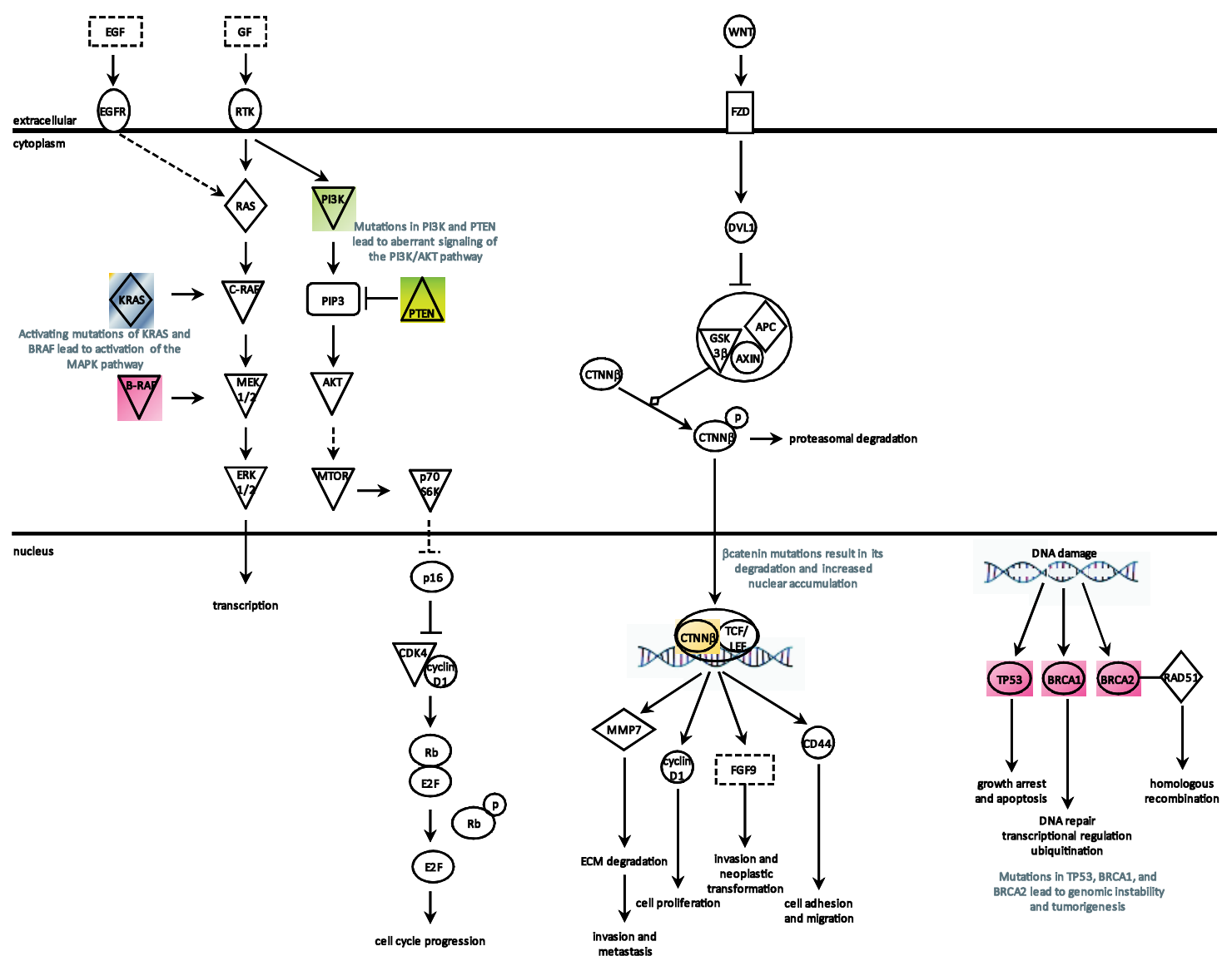

Figure 1: Schematic representation of altered signaling pathways in ovarian cancer

Red represents genes altered in serous tumors; green: clear cell carcinomas; orange: endometrioid carcinomas; combination of yellow and green: endometrioid and clear cell carcinomas, grey represents both serous and mucinous tumors 
Table S2: Primer sequences quantitative RT-PCR and TP53 mutation analysis

\begin{tabular}{|c|c|c|c|c|c|}
\hline Gene & Gene symbol & NM transcript ID & Unigene ID & Direction & Sequence (5' - 3') \\
\hline Cyclin E1 & CCNE1 & NM_001238 /// NM_057182 & Hs.244723 & $\begin{array}{l}\text { Forward } \\
\text { Reverse }\end{array}$ & $\begin{array}{l}\text { CGACAGGACGGCGAGGG } \\
\text { GGTAAACCCGGTCATCATCTTC }\end{array}$ \\
\hline E2F1 & E2F1 & NM_005225 & Hs.654393 & $\begin{array}{l}\text { Forward } \\
\text { Reverse }\end{array}$ & $\begin{array}{l}\text { GGAGAAGTCACGCTATGAGACCTC } \\
\text { GCCGCTTCTGCACCTTCAG }\end{array}$ \\
\hline E2F3 & E2F3 & NM_001949 & Hs.269408 & $\begin{array}{l}\text { Forward } \\
\text { Reverse }\end{array}$ & $\begin{array}{l}\text { CAACTCAGGACATAGCGATTGCT } \\
\text { ACGGTCCTTCTAGGTTGGAAGG }\end{array}$ \\
\hline p21 (WAF1/CIP1) & CDKN1A & NM_000389 /// NM_078467 & Hs.370771 & $\begin{array}{l}\text { Forward } \\
\text { Reverse }\end{array}$ & $\begin{array}{l}\text { GACCATGTGGACCTGTCACTGTC } \\
\text { TCTGTCATGCTGGTCTGCCG }\end{array}$ \\
\hline Actin, Beta & ACTB & NM_001101 & Hs.520640 & $\begin{array}{l}\text { Forward } \\
\text { Reverse }\end{array}$ & $\begin{array}{l}\text { GCTGTGCTACGTCGCCCTG } \\
\text { GGAGGAGCTGGAAGCAGCC }\end{array}$ \\
\hline p53 & TP53 & NM_000546 /// NM_001126112-7 & Hs.654481 & & \\
\hline Exon 5 & & & & $\begin{array}{l}\text { Flank forward } \\
\text { Flank reverse } \\
\text { Inside forward }\end{array}$ & $\begin{array}{l}\text { CTGTTCACTTGTGCCCTGAC } \\
\text { AGCCCTGTCGTCTCTCCAG } \\
\text { TGCCCTGACTTTCAACTCTG }\end{array}$ \\
\hline Exon 6 & & & & $\begin{array}{l}\text { Flank forward } \\
\text { Flank reverse } \\
\text { Inside reverse }\end{array}$ & $\begin{array}{l}\text { GGCCTCTGATTCCTCACTG } \\
\text { ATAAGCAGCAGGAGAAAGCC } \\
\text { GACCCCAGTTGCAAACCAG }\end{array}$ \\
\hline Exon 7 & & & & $\begin{array}{l}\text { Flank forward } \\
\text { Flank reverse } \\
\text { Inside reverse }\end{array}$ & $\begin{array}{l}\text { CATCTTGGGCCTGTGTTATC } \\
\text { GATGTGATGAGAGGTGGATGG } \\
\text { GGAAGAAATCGGTAAGAGGTG }\end{array}$ \\
\hline Exon 8 & & & & $\begin{array}{l}\text { Flank forward } \\
\text { Flank reverse } \\
\text { Inside reverse }\end{array}$ & $\begin{array}{l}\text { GATTTCCTTACTGCCTCTTGC } \\
\text { CATAACTGCACCCTTGGTCTC } \\
\text { TCCTCCACCGCTTCTTGTC }\end{array}$ \\
\hline
\end{tabular}




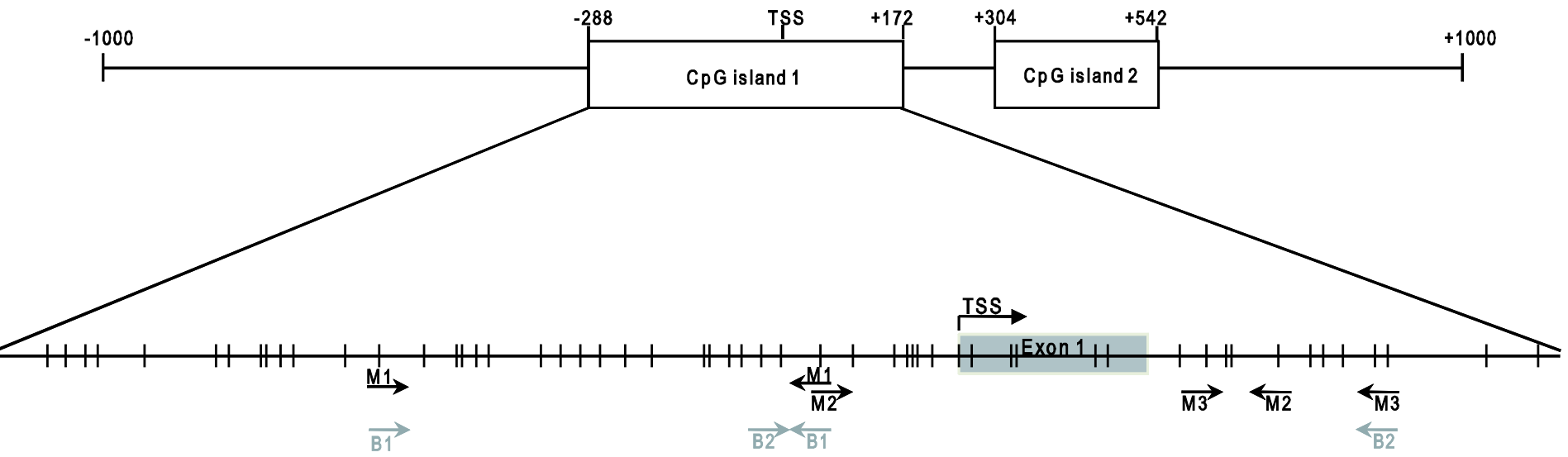

B

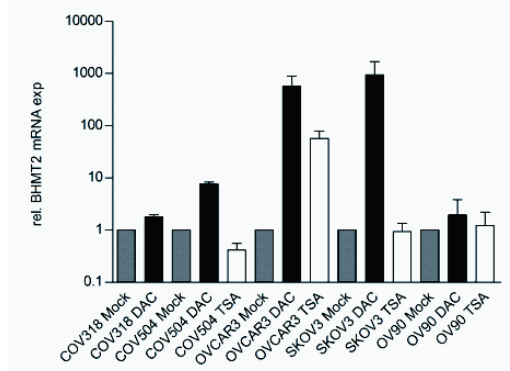

C

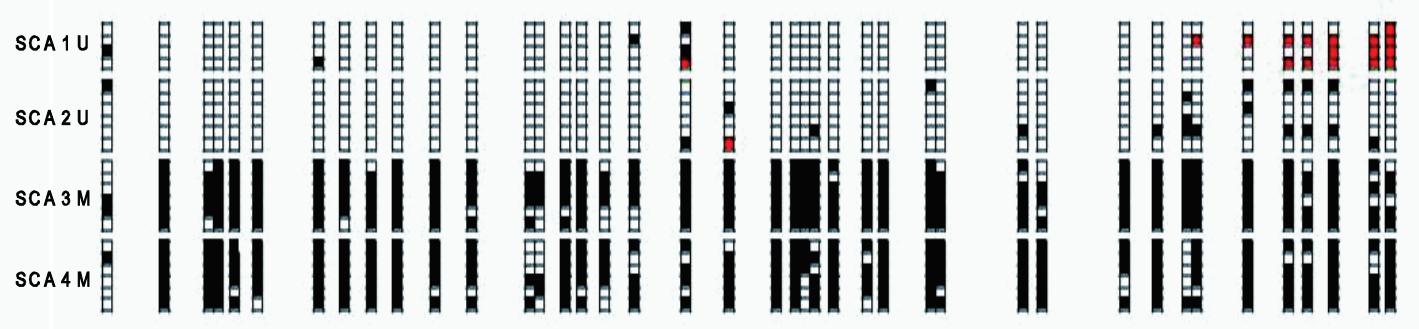

Figure 2: BHMT2 promoter and mRNA expression in ovarian cancer cell lines, and bisulfite sequencing in SCAs

(A) BHMT2 promoter showing the two CpG islands. TSS: transcription start site; MSP primers M1 (-192 to -37 relative to TSS), M2 (-50 to +10), M3 (+43 to +152); bisulfite sequencing primers B1 $(-192$ to -35$)$ and B2 $(-63$ to +154$)$. Each vertical line represents a CpG dinucleotide. Panel (B) shows BHMT2 mRNA expression in ovarian cancer cell lines after mock, DAC and TSA treatment. ${ }^{*} \mathrm{p} \leq 0.05$. (C) To verify methylation, bisulfite sequencing of four SCAs (2 unmethylated (SCA1 and SCA2) and 2 methylated (SCA3 and SCA4)) was performed. Each row represents an individual cloned allele. Each box represents a $\mathrm{CpG}$ dinucleotide; black boxes represent methylated $\mathrm{CpGs}$, white unmethylated $\mathrm{CpGs}$, and red not-interpretable results. 
Supplementary Table S1: MSP, BST sequencing and quantitative RT-PCR primers

\begin{tabular}{|c|c|c|c|c|c|c|}
\hline Analysis & Primer & Forward primer (5'-3') & Reverse primer (5'-3') & Amplicon (bp) & $\begin{array}{l}\text { Annealing } \\
\text { temp }\left({ }^{\circ} \mathrm{C}\right)\end{array}$ & $\begin{array}{l}\text { No of PCR } \\
\text { cycles }\end{array}$ \\
\hline \multirow[t]{9}{*}{ MSP } & Flank 1 & GGGTAGTTTYGTAGTAGGAGGTTT & CCAACCCRAAAAAAAAATCC & 156 & 56 & 35 \\
\hline & U1 & TTTTGTAGTAGGAGGTTTTGTAGTAGTTGT & САААAАAААААТССААСССССА & 144 & 66 & 30 \\
\hline & M1 & CGTAGTAGGAGGTTTCGTAGTAGTTGC & GAAAAAAAAATCCGACCCCCG & 140 & 66 & 30 \\
\hline & Flank 2 & TTTTTTYGGGTTGGGGG & ACCCAACRACCAAAAACTCC & 152 & 56 & 35 \\
\hline & & GGGTGGGTTTTTAAGATGGTTGT & AААСТССАСАААААТСААААAАACA & 125 & 64 & 35 \\
\hline & M2 & CGGGTTTTTAGACGGTCGC & CGCGAAAATCGAAAAAACG & 116 & 64 & 35 \\
\hline & Flank 3 & GGGGGTTAAGAAGGTGAGTTT & ССССААААСТТААСТАААААТАААС & 110 & 56 & 35 \\
\hline & U3 & AGGTGAGTTTTGTTTTTTTTGATTTTT & 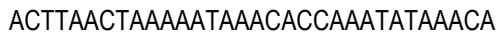 & 92 & 66 & 25 \\
\hline & M3 & GTGAGTTTCGTTTTTTTCGATTTTC & AAAAATAAACGCCGAATATAAACG & 82 & 66 & 25 \\
\hline \multirow[t]{2}{*}{ BST seq } & B1 & GGGTAGTTTYGTAGTAGGAGGTTT & CCCCAACCCRAAAAAAAA & 158 & 58 & 40 \\
\hline & B2 & GGGGGTYGGATTTTTTTTTT & ААССССАAААСТТААСТАААААТАА & 218 & 60 & 40 \\
\hline \multirow[t]{3}{*}{ RT-PCR } & BHMT2 & CGCCTGGAGAGTGGGGAG & CCTGCTCTCAAGAATTCCATGTG & 155 & & \\
\hline & $\beta$-actin & GCTGTGCTACGTCGCCCTG & GGAGGAGCTGGAAGCAGCC & 61 & & \\
\hline & cyclophilin A & CTCGAATAAGTTTGACTTGTGTTT & CTAGGCATGGGAGGGAACA & 165 & & \\
\hline
\end{tabular}

